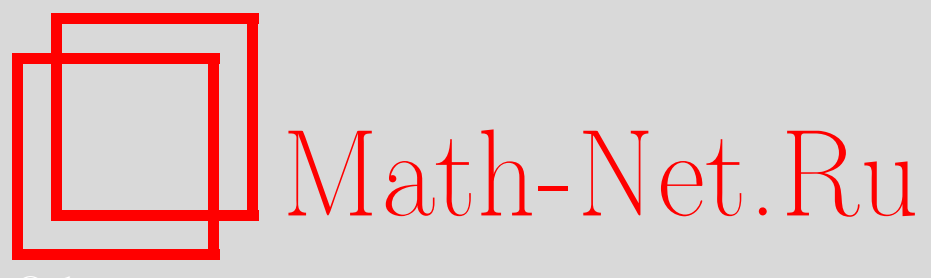

К. Болдригини, Р. А. Минлос, А. Пеллегринотти, Случайные блуждания в случайной (флуктуирующей) среде, УМH, 2007, том 62, выпуск 4, 27-76

DOI: https://doi.org/10.4213/rm6849

Использование Общероссийского математического портала Math-Net.Ru подразумевает, что вы прочитали и согласны с пользовательским соглашением http://www.mathnet.ru/rus/agreement

Параметры загрузки:

IP : 54.172 .240 .79

26 апреля 2023 г., 16:05:11

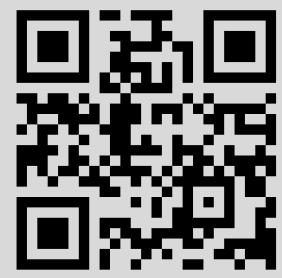


УДК 519.21

\section{Случайные блуждания в случайной (флуктуирующей) среде}

\section{К. Болдригини, Р. А. Минлос, А. Пеллегринотти}

Основное содержание статьи заключено в доказательстве центральной предельной теоремы для положения на решетке $\mathbb{Z}^{d}$ частицы, взаимодействующей со случайной "средой” в течение длительного промежутка времени (и выходящей из фиксированной точки решетки). Рассмотрены два случая: конфигурация "среды” (т. е. случайного поля) фиксирована во всех точках "пространства-времени" $\mathbb{Z}^{d+1}$ (так называемая quenchedмодель) или же поле изменяется со временем вместе с положением частицы так, что пара (поле+частица) образует марковскую цепь (annealedмодель). Для quenched-моделей изучены два случая: значения поля во всех точках "пространства-времени" независимы и одинаково распределены или же значения поля связаны в однородную марковскую цепь. При этом центральная предельная теорема с одним и тем же предельным законом верна в случае quenched-моделей для почти всех конфигураций "среды", а в случае annealed-моделей для любого начального распределения поля. Кроме центральной предельной теоремы в статье затронуты кратко и некоторые другие темы, касающиеся упомянутых моделей (убывание корреляций, большие уклонения, "поле с точки зрения частицы" и т. д.).

Библиография: 25 названий.

\section{СОДЕРЖАНИЕ}

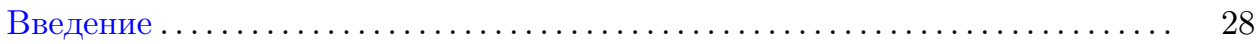

$\S 1$. Блуждание в независимом поле; quenched-модель . . . . . . . . . . . 32

1.1. Усредненная модель и основной результат.............. 32

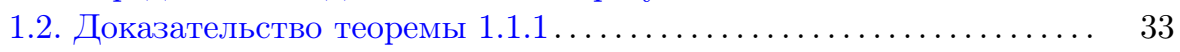

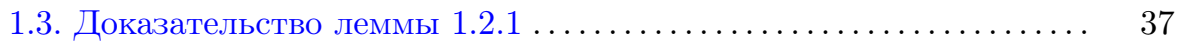

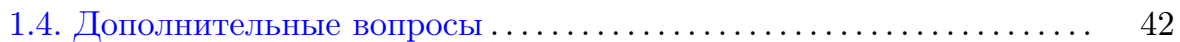

1.4.1. Другие подходы ............................. 42

1.4.2. Поправка к гауссовскому члену в асимптотике $\mu_{T}(f ; \eta)$ в од-

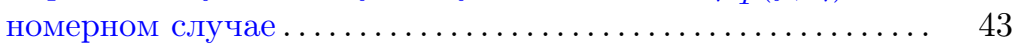

1.4.3. Локальная предельная теорема .................. 44

Первый и третий авторы поддержаны фондами INdAM (C.N.F.M.) и M.U.R.S.T. (PRIN); второй автор поддержан РФФИ (грант № 05-01-00449) и CRDF (грант RUM1-2693-MO-05). Все авторы выражают благодарность Г. Н. Давыдовой за неоценимую помощь в подготовке рукописи к печати.

(C) К. Болдригини, Р. А. Минлос, А. ПЕллегринотти, 2007 
$\S 2$. Блуждание в марковской среде; annealed-модель.................. 44

2.1. Центральная предельная теорема для положения частицы..... 44

2.2. Доказательство теоремы 2.1.1; приготовления............. 46

2.3. Завершение доказательства теоремы $2.1 .1 \ldots \ldots \ldots \ldots \ldots \ldots \ldots . \ldots 1$

2.4. Другие темы (иной метод доказательства; поле с точки зрения частицы; убывание корреляций; большие уклонения) ........ 52

$\S 3$. Блуждание в марковском поле; quenched-модель ............... 55

3.1. Усредненная модель и формулировка основного результата.... 55

3.2. Начало доказательства теоремы 3.1.1; основные конструкции... 56

3.2.1. Мультипликативный базис .................... 56

3.2.2. Разложение вероятности $\operatorname{Pr}\left(\chi_{T} ; \eta\right)$ по базису $\left\{V_{B}\right\} \ldots \ldots .57$

3.2.3. Окончательное представление для $\operatorname{Pr}\left(X_{T}=y \mid X_{0}=0 ; \eta\right) . \quad 58$

3.3. Завершение доказательства теоремы $3.1 .1 \ldots \ldots \ldots \ldots \ldots \ldots \ldots .61$

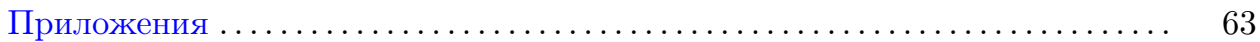

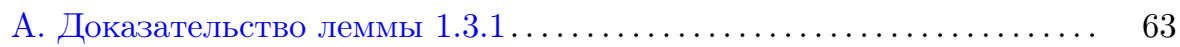

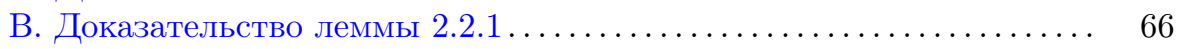

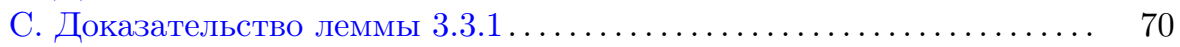

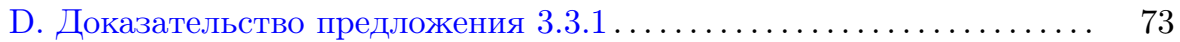

Список литературы ......................................... 74

\section{Введение}

В настоящей статье приводится обзор многолетнего изучения моделей случайных блужданий частицы в случайной (флуктуирующей во времени) среде. Цель этого обзора - познакомить читателя с этой новой и малоизвестной тематикой, а также с техникой, которая была выработана в связи с этими исследованиями.

Опишем в общих чертах эти модели. "Среда" задается случайным стационарным полем

$$
\eta=\left\{\eta(x, t), x \in \mathbb{Z}^{\nu}, t \in \mathbb{Z}^{1}\right\},
$$

определенным на $(\nu+1)$-мерной решетке $\mathbb{Z}^{\nu} \times \mathbb{Z}^{1}$ ("пространство-время") и принимающим значения из некоторого конечного множества $S$. Наиболее интересный класс моделей - это модели с “замороженной” в каждый момент времени средой. Мы предполагаем, что конфигурация поля $\eta(x, t)$ фиксирована, и рассматриваем "марковское" блуждание (вообе говоря, неоднородное) частицы по решетке $\mathbb{Z}^{\nu}$ в такой среде, начинающееся при $t=0$ в некоторой фиксированной точке $x_{0} \in \mathbb{Z}^{\nu}{ }^{1}$ При этом вероятность перескока частицы за один такт времени из положения $X_{t}$ в положение $X_{t+1}$ зависит от значения поля $\eta\left(X_{t}, t\right)$ в точке $X_{t}$ в момент $t \geqslant 0$ :

$$
\operatorname{Pr}\left(X_{t+1}-X_{t}=u \mid X_{t}=x ; \eta\right)=p(u ; \eta(x, t)),
$$

где функция $p(u ; s), u \in \mathbb{Z}^{\nu}$, при каждом фиксированном значении $s \in S$ задает вероятностное распределение на решетке $\mathbb{Z}^{\nu}$. Обозначим

$$
p_{0}(u)=\langle p(u ; \eta(x, t))\rangle_{\eta}=\sum_{s \in S} p(u ; s) \pi(s) .
$$

\footnotetext{
${ }^{1}$ Подчеркнем, что конфигурация среды $\eta(x, t)$ мыслится меняющейся со временем (флуктуирует). Иногда рассматривают случай среды, “замороженной” в начальный момент и остающейся неизменной в дальнейшем (см. [1]). Мы не рассматриваем здесь эту задачу.
} 
Здесь скобки $\langle\cdot\rangle_{\eta}$ означают среднее по распределению поля $(0.1)$, а $\pi(s)$, $s \in S,-$ распределение вероятностей для значения поля $\eta$ в любой точке $(x, t) \in \mathbb{Z}^{\nu} \times \mathbb{Z}^{1}$ (в силу стационарности поля это распределение одинаково для всех точек $(x, t))$. Очевидно, что функция $p_{0}(u), u \in \mathbb{Z}^{\nu}$, также задает распределение вероятностей на решетке $\mathbb{Z}^{\nu}$. Запишем, далее,

$$
p(u ; s)=p_{0}(u)+c(u ; s) .
$$

Очевидно, что

$$
\begin{gathered}
\sum_{u \in \mathbb{Z}^{\nu}} c(u ; s)=0, \quad s \in S, \\
\langle c(u ; \cdot)\rangle_{\eta}=\sum_{s} c(u ; s) \pi(s)=0, \quad u \in \mathbb{Z}^{\nu} .
\end{gathered}
$$

Далее мы предполагаем, что

i) функция $p(u ; s)$, а тем самым и функции $p_{0}(u), c(u ; s)$ финитны относительно переменного $u$ :

$$
p(u ; s)=p_{0}(u)=c(u ; s)=0
$$

при всех $s$, если $|u|=\sum_{j=1}^{n}\left|u_{j}\right|>D$, где $D>0$ - некоторая константа, a $u=\left\{u_{1}, \ldots, u_{\nu}\right\} \in \mathbb{Z}^{\nu}$

ii) характеристическая функция распределения $p_{0}$

$$
\tilde{p}_{0}(\lambda)=\sum_{u \in \mathbb{Z}^{\nu}} p_{0}(u) e^{i(u, \lambda)},
$$

где $\lambda=\left(\lambda_{1}, \ldots, \lambda_{\nu}\right)$ принадлежит $\nu$-мерному тору $\mathbb{T}^{\nu}$, удовлетворяет условию

$$
\left|\tilde{p}_{0}(\lambda)\right|<1, \quad \lambda \neq 0
$$

Обозначим через

$$
\chi_{T}=\left\{X_{0}, X_{1}, \ldots, X_{T}\right\}
$$

начальный отрезок траектории блуждающей частицы за $T$ шагов, $X_{k} \in \mathbb{Z}^{\nu}$, $k=0,1, \ldots, T$.

Из (0.2) и (0.4) видно, что при фиксированной начальной точке $X_{0}=x_{0}$ вероятность того, что начальный отрезок траектории $\chi_{T}$ имеет вид $(0.7 \mathrm{~b})$, равна

$$
\begin{aligned}
\operatorname{Pr}\left(\chi_{T} \mid X_{0}=x_{0} ; \eta\right) & =\prod_{t=0}^{T-1} p\left(X_{t+1}-X_{t} ; \eta\left(X_{t}, t\right)\right) \\
& =\prod_{t=0}^{T-1}\left(p_{0}\left(X_{t+1}-X_{t}\right)+c\left(X_{t+1}-X_{t} ; \eta\left(X_{t}, t\right)\right)\right) .
\end{aligned}
$$

При этом вероятность того, что частица в момент $T$ окажется в точке $y$, равна

$$
\operatorname{Pr}\left(X_{T}=y \mid X_{0}=x_{0} ; \eta\right)=\sum_{\chi_{T}: X_{0}=x_{0}, X_{T}=y} \operatorname{Pr}\left(\chi_{T} \mid X_{0}=x_{0} ; \eta\right) .
$$


Основная задача, разбираемая в этом обзоре, это асимптотика распределения $(0.8)$ при $T \rightarrow \infty$. По-видимому, если случайные поля достаточно регулярны, то для распределения (0.8) верна центральная предельная теорема с одними и теми же параметрами при почти всех реализациях поля $\eta$. Это означает следующее: существуют такой вектор $b \in \mathbb{R}^{\nu}$ ("снос") и такая положительно-определенная матрица $A=\left\{a_{i j}\right\}$, что для любой ограниченной области $G \subset \mathbb{R}^{\nu}$ с кусочно гладкой границей имеет место сходимость

$$
\operatorname{Pr}\left(X_{T}-X_{0} \in \sqrt{T} G+b T \mid X_{0}=x_{0} ; \eta\right) \rightarrow \int_{G} g(u) d u, \quad T \rightarrow \infty
$$

при почти всех $\eta \in \Omega$ (или же в метрике какого-нибудь из пространств $L_{p}(\Omega, \rho)$, $p \geqslant 1, \Omega=S^{\mathbb{Z}^{\nu+1}}, \rho$ - распределение вероятностей поля $\left.\eta\right)$. Здесь

$$
g(u)=\frac{(\operatorname{det} A)^{1 / 2}}{(2 \pi)^{\nu / 2}} e^{-\frac{1}{2} \mathscr{A}(u)}, \quad u=\left(u_{1}, \ldots, u_{\nu}\right) \in \mathbb{R}^{\nu},
$$

- гауссова плотность, $\mathscr{A}(u)=\sum_{i, j} a_{i j} u_{i} u_{j}$ - квадратичная форма с матрицей $A=\left\{a_{i j}\right\}$, а $\sqrt{T} G+b T$ - область, получаемая растяжением области $G$ в $\sqrt{T}$ раз с последующим сдвигом на вектор $b T$.

В настоящем обзоре гипотеза (0.9) проверена для случая независимого поля $\eta$, а также для некоторого класса марковских полей (в последнем случае предполагается, что влияние поля на блуждание частицы достаточно мало, т. е. функция $c(u ; s)$ в (0.4) мала); см. работы [2]-[4]. Описанный выше класс моделей, т. е. блуждание частицы в фиксированной в каждый момент времени случайной среде, в англоязычной математической литературе обычно обозначается словом quenched, и мы будем для краткости называть такие модели quenched-моделями. Для каждой quenched-модели определена так называемая "усредненная" модель случайного блуждания частицы: при фиксированном начальном положении $X_{0}=x_{0}$ частицы вероятность начального отрезка траектории $\chi_{T}$ за $T$ шагов в усредненной модели определяется как среднее по полю от вероятностей $(0.7 \mathrm{c})$ :

$$
\operatorname{Pr}^{\text {ycp. }}\left(\chi_{T} \mid X_{0}=x_{0}\right)=\left\langle\operatorname{Pr}\left(\chi_{T} \mid X_{0}=x_{0} ; \eta\right)\right\rangle_{\eta} .
$$

Здесь будет также рассмотрен и другой класс моделей, описывающих блуждание частицы, взаимодействующей со случайной средой. В этих моделях принимается, что пары $\left(X_{t}, \eta_{t}\right), t \geqslant 0$, состоящие из $X_{t}$ - положения блуждающей частицы в момент $t$ и конфигурации $\eta_{t}=\left\{\eta(x, t), x \in \mathbb{Z}^{\nu}\right\}$, образуют марковскую цепь и при этом выполнено:

1) при фиксированной в момент $t$ паре $\left(X_{t}=\bar{x}, \eta_{t}=\bar{\eta}\right)$ положение частицы $X_{t+1}$ и значения поля $\left\{\eta_{t+1}(x), x \in \mathbb{Z}^{1}\right\}$ в различных точках решетки в следующий момент времени условно независимы;

2) вероятность скачка частицы $X_{t} \rightarrow X_{t+1}$ по-прежнему имеет вид $(0.4)$ и для функций $p_{0}(u)$ и $c(u ; s)$ выполнены все перечисленные выше (см. условия і) и іi)) требования;

3) условное распределение для значения поля $\eta_{t+1}(z)$ в точке $z \in \mathbb{Z}^{\nu}$ имеет вид

$$
\operatorname{Pr}\left(\eta_{t+1}(z)=s \mid X_{t}=\bar{x}, \eta_{t}=\bar{\eta}\right)=q_{\bar{x}-z}(\bar{\eta}(z), s),
$$


где $\left\{q_{u}\left(s^{\prime}, s\right)\right\}_{s^{\prime}, s \in S}=Q_{u}, u \in \mathbb{Z}^{\nu}$, - семейство стохастических матриц такое, что при $|u| \geqslant d$ это семейство стабилизируется:

$$
Q_{u}=\widehat{Q}, \quad|u| \geqslant d
$$

где $d \geqslant 0$ - некоторая константа, а $\widehat{Q}$ - фиксированная стохастическая матрица.

Такой выбор переходных вероятностей (0.11) означает, что в момент времени $t$ частица влияет на изменение поля лишь в тех точках $z$, которые отстоят от нее на расстояние, меньшее $d$. В частности, при $d=0$ поле эволюционирует в соответствии с матрицей $\widehat{Q}$ "само по себе" - независимо от блуждающей частицы. Заметим, что в этом последнем случае усредненная по полю вероятность траектории частицы совпадает с такой же усредненной вероятностью, вычисленной для соответствующей quenched-модели. Этим обстоятельством мы воспользуемся в последнем параграфе статьи.

Этот второй класс моделей в западной литературе обозначается словом "annealed", и мы ради краткости будем говорить об annealed-моделях ${ }^{2}$. В этом случае снова возникает вопрос об асимптотике распределения (0.8) положения частицы $X_{T}$ при больших $T$ и, по-видимому, для широкого класca annealedмоделей также верна центральная предельная теорема, т. е. асимптотика (0.9).

Помимо главной темы - асимптотики (0.9) - мы в этом обзоре упомянем несколько смежных сюжетов, касающихся описанных моделей: следующие члены в разложении для центральной предельной теоремы, асимптотики (или же оценки убывания) корреляций для разного рода характеристик модели, “поле с точки зрения частицы", большие уклонения для распределения (0.8) и т. д.

Статья построена следующим образом: в 11 мы занимаемся quenched-моделью для случая независимого поля на решетке. Здесь мы формулируем центральную предельную теорему, имея в виду два вида сходимости: сходимость в метрике $L_{2}$ и поточечную сходимость для почти всех конфигураций поля. Доказательство - с тем чтобы не усложнять чрезмерно изложение - приводится лишь для случая $L_{2}$-сходимости. В $\S 2$ изучается простейшая annealed-модель и доказывается локальная предельная теорема для распределения $\operatorname{Pr}\left(X_{T}=y \mid X_{0}=x_{0}\right)$ при $T \rightarrow \infty$. В $\S 3$ мы рассматриваем quenchedмодель для марковского поля. Здесь, как и в $\S 1$, центральная предельная теорема формулируется для обоих видов сходимости (почти всюду и в метрике $L_{2}$ ), но доказывается - по тем же причинам, что и выше - только для случая $L_{2}$-сходимости. Доказательства ряда лемм чисто технического характера вынесены в приложение.

Отметим в заключение, что тематика данного обзора близка к задачам о так называемых направленных полимерах в случайной среде [5]. Направленный полимер в пространстве $\mathbb{R}^{\nu+1}$ (или на решетке $\mathbb{Z}^{\nu+1}$ ) - это случайная нить, вытянутая вдоль одного из фиксированных направлений (которое во всех моделях ассоциируется со “временем") и представляемая как траектория случайного блуждания. Каждой такой траектории (полимеру) сопоставляется вес, похожий на выражение $(0.7 \mathrm{c})$, зависящий от случайной среды и задающий при

\footnotetext{
${ }^{2}$ Иногда под annealed-моделью разумеют просто усредненную модель (0.10).
} 
надлежащей нормировке вероятность траектории. Из этого краткого пояснения понятно, что математические приемы исследования, выработанные в задачах о случайном блуждании в случайной среде, применимы и в моделях направленных полимеров.

\section{§ 1. Блуждание в независимом поле; quenched-модель}

1.1. Усредненная модель и основной результат. Предположим, что значения поля (0.1) в разных точках "пространства-времени" $\mathbb{Z}^{\nu} \times \mathbb{Z}^{1}$ независимы и имеют одинаковые распределения $\{\pi(s), s \in S\}$. Как следует из (0.7c) и $(0.5 \mathrm{~b})$, для усредненной модели $(0.10)$ в этом случае вероятность траектории $\chi_{T}(0.7 \mathrm{~b})$ имеет вид

$$
\operatorname{Pr}^{\mathrm{ycp} .}\left(\chi_{T} \mid X_{0}=x_{0}\right)=\prod_{t=0}^{T-1} p_{0}\left(X_{t+1}-X_{t}\right) .
$$

Иными словами, усредненная модель в случае независимого поля является просто моделью однородного случайного блуждания с вероятностями перескока $\operatorname{Pr}\left(X_{t} \rightarrow X_{t+1}\right)=p_{0}\left(X_{t+1}-X_{t}\right)$. При этом условие $(0.7$ a) означает, что такое блуждание вполне неприводимо (см. [6]). В этом случае, как известно, для вероятностей $\operatorname{Pr}^{\text {уср. }}\left(X_{T}=y \mid X_{0}=x_{0}\right)$ верна центральная предельная теорема (0.9a) (см. [6]). Вектор $b$ и матрица $A=\left\{a_{j k}\right\}$ находятся по следующим формулам:

$$
\begin{gathered}
b=\left.i \nabla \log \tilde{p}_{0}(\lambda)\right|_{\lambda=0}, \\
A^{-1}=C=\left\{c_{j k}\right\}, \quad c_{j k}=-\left.\frac{\partial^{2} \log \tilde{p}_{0}(\lambda)}{\partial \lambda_{j} \partial \lambda_{k}}\right|_{\lambda=0},
\end{gathered}
$$

где $\lambda=\left(\lambda_{1}, \ldots, \lambda_{\nu}\right) \in \mathbb{T}^{\nu}$, а $\tilde{p}_{0}(\lambda)$ - характеристическая функция распределения $p_{0}$ (см. (0.6)).

Мы предполагаем, что кроме условий і) и іi) выполнено условие

iii) матрица $C$ положительно определена.

Переходя к полной quenched-модели с независимым полем $\eta$, мы введем еще одно требование относительно вероятности (0.2). Пусть $\tilde{p}(\lambda ; s)$ - характеристическая функция этой переходной вероятности:

$$
\tilde{p}(\lambda ; s)=\sum_{x \in \mathbb{Z}^{\nu}} e^{i(x, \lambda)} p(x ; s) .
$$

Очевидно, что при всех $s$ выполнено неравенство $|\tilde{p}(\lambda ; s)| \leqslant 1$ при любом $\lambda \in \mathbb{T}^{\nu}$. Если при некотором $s_{0} \in S$ имеет место тождество

$$
\left|\tilde{p}\left(\lambda ; s_{0}\right)\right| \equiv 1
$$

при всех $\lambda \in \mathbb{T}^{\nu}$, то это значит, что распределение $p\left(x ; s_{0}\right)$ сосредоточено в какой-то одной точке $u_{0}=u_{0}\left(s_{0}\right) \in \mathbb{Z}^{\nu}$ :

$$
p\left(u_{0}\left(s_{0}\right) ; s_{0}\right)=1 \text {. }
$$

Если такое случается при всех $s \in S$ и $\lambda \in \mathbb{T}^{\nu}$, т. е.

$$
|\tilde{p}(\lambda ; s)| \equiv 1
$$


то блуждание при фиксированной конфигурации поля $\eta$ оказывается детерминированным: попав в точку $x$ в момент времени $t$, частица с вероятностью 1 попадет в следующий момент в точку $x+u_{0}(\eta(x, t))$. Мы отбросим такой случай, т. е. будем считать, что величина $|\tilde{p}(\lambda ; s)|$ не равна тождественно 1 при всех $\lambda$ и $s$. Нам удобно представить это требование в следующей эквивалентной форме:

iv) выполнено неравенство

$$
\int_{\mathbb{T}^{\nu}}\left\langle|\tilde{p}(\lambda ; \cdot)|^{2}\right\rangle_{\eta} d m(\lambda)=\int_{\mathbb{T}^{\nu}}\left(\sum_{s \in S}|\tilde{p}(\lambda ; s)|^{2} \pi(s)\right) d m(\lambda)<1,
$$

где $d m(\lambda)=\frac{d^{\nu} \lambda}{(2 \pi)^{\nu}}-$ нормированная мера Хаaра на торе $\mathbb{T}^{\nu}$.

Итак, основной результат данного параграфа заключен в следующей теореме.

ТеОРема 1.1.1. Пусть выполнены условия i)-iv). Тогда при всех $\nu \geqslant 1$ для случайного блуждания частицы в независимом одинаково распределенном поле, начинающегося в точке $x_{0} \in \mathbb{Z}^{\nu}$, верно утверждение (0.9). При этом вектор $b$ и матрица А определены формулами (1.1.2). Сходимость в (0.9) понимается как сходимость для почти всех конфигураций $\eta \in \Omega$, а также как сходимость в метрике пространства $L_{2}(\Omega, \rho)$ :

$$
\left\|\operatorname{Pr}\left(X_{T}-X_{0} \in \sqrt{T} G+b T \mid X_{0}=x_{0} ; \eta\right)-\int_{G} g(u) d u\right\|_{L_{2}} \rightarrow 0,
$$

при $T \rightarrow \infty$ для любой ограниченной области $G \subset \mathbb{R}^{\nu}$ с кусочно гладкой границей.

1.2. Доказательство теоремы 1.1.1. Далее, как было сказано во введении, мы докажем только утверждение (1.1.5). Полное доказательство теоремы 1.1.1 можно найти в [7]. Ниже, не теряя общности, будем считать, что $x_{0}=0$, и в дальнейших обозначениях будем опускать букву $x_{0}$. Условимся также обозначать различные постоянные, встречающиеся в тексте, одной и той же буквой $C$.

Раскрыв скобки в $(0.7 \mathrm{c})$, мы получим, что

$$
\operatorname{Pr}\left(\chi_{T} ; \eta\right)=P_{0}^{\text {ycp. }}\left(\chi_{T}\right)+\sum_{\substack{B \subseteq \chi_{T} \\ B \neq \varnothing}} \prod_{X_{t} \in B} c\left(X_{t+1}-X_{t} ; \eta\left(X_{t}, t\right)\right) \prod_{X_{t} \notin B} p_{0}\left(X_{t+1}-X_{t}\right),
$$

где $P_{0}^{\text {уср. }}$ - вероятность траектории в усредненной модели (см. (1.1.1)), а $B=$ $\left\{\left(y_{1}, t_{1}\right),\left(y_{2}, t_{2}\right), \ldots,\left(y_{k}, t_{k}\right)\right\} \subseteq \chi_{T}, 0 \leqslant t_{1}<t_{2}<\cdots<t_{k} \leqslant T-1$. Для каждого слагаемого в (1.2.1) точки $(y, t) \in \chi_{T}$, не попавшие в множество $B$, назовем свободными точками. Далее, подставляя (1.2.1) в формулу (0.8) и суммируя по всем свободным точкам траектории при фиксированном $B$, получим, что

$$
\begin{aligned}
& \operatorname{Pr}\left(X_{T}=y ; \eta\right)=P_{0}^{T}(y)+\sum_{\substack{B=\left\{\left(y_{1}, t_{1}\right), \ldots,\left(y_{k}, t_{k}\right)\right\} \\
0 \leqslant t_{1}<t_{2}<\cdots<t_{k} \leqslant T-1}} P_{0}^{t_{1}}\left(y_{1}\right) \\
& \quad \times \prod_{s=1}^{k-1} h^{t_{s+1}-t_{s}}\left(y_{s+1}-y_{s} ; \eta\left(y_{s}, t_{s}\right)\right) h^{T-t_{k}}\left(y-y_{k} ; \eta\left(y_{k}, t_{k}\right)\right) .
\end{aligned}
$$


Здесь использованы обозначения $P_{0}^{t}(y)=\left(p_{0} * p_{0} * \cdots * p_{0}\right)(y)(t$-кратная свертка, $t>0), P_{0}^{0}(y)=\delta_{y, 0}($ символ Кронекера) и

$$
h^{t}(y ; s)=\left(c(\cdot ; s) * P_{0}^{t-1}(\cdot)\right)(y), \quad t>0 .
$$

Вывод формулы (1.2.2) наглядно пояснен на рис. 1.

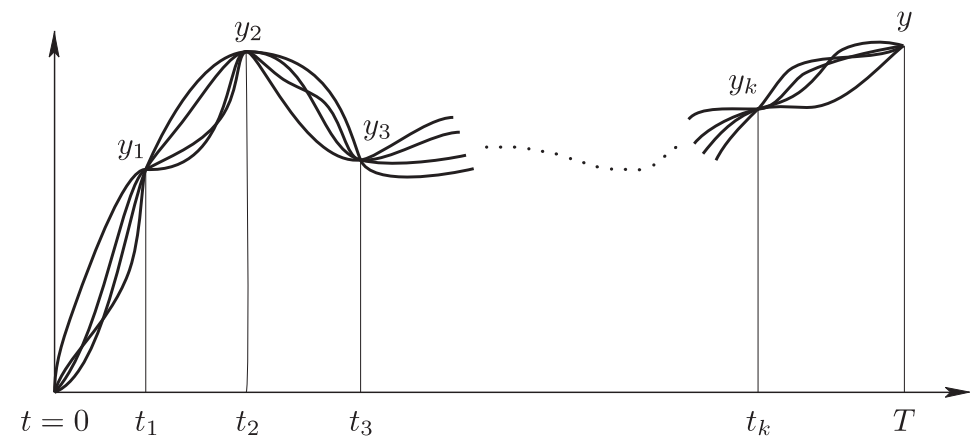

Рис. 1. Множество $B=\left\{\left(y_{1}, t_{1}\right), \ldots,\left(y_{k}, t_{k}\right)\right\}$; суммирование по свободным точкам траектории $\chi_{T}$ с одним и тем же множеством $B$, т. е. суммирование по отрезкам траекторий между последовательными точками из $B$

Введем обозначения

$$
M_{B}(\eta)=P_{0}^{t_{1}}\left(y_{1}\right) \prod_{s=1}^{k-1} h^{t_{s+1}-t_{s}}\left(y_{s+1}-y_{s} ; \eta\left(t_{s}, y_{s}\right)\right)
$$

и

$$
\mathscr{M}(z, t ; \eta)=\sum_{\substack{B: t_{f}(B)=t \\ y_{f}(B)=z}} M_{B}(\eta)
$$

где $\left(y_{f}, t_{f}\right)=\left(y_{k}, t_{k}\right)$ - последняя по времени точка $B$. Окончательно получим, что

$$
\operatorname{Pr}\left(X_{T}=y ; \eta\right)=P_{0}^{T}(y)+\sum_{t=0}^{T-1} \sum_{z \in \mathbb{Z}^{\nu}} \mathscr{M}(z, t ; \eta) h^{T-t}(y-z ; \eta(z, t)) .
$$

Доказательство утверждения (1.1.5) основано на следующей асимптотике, доказанной ниже (см. раздел 1.3).

Лемма 1.2.1. Для всех $\nu \geqslant 1$ верно следующее асимптотическое соотношение: $п$ ри $t \rightarrow \infty$

$$
\sum_{z \in \mathbb{Z}^{\nu}}\left\langle\mathscr{M}^{2}(z, t ; \eta)\right\rangle_{\eta}=C t^{-\nu / 2}(1+o(1))
$$

где $C>0$ - некоторая константа. 
Обозначим через $C_{\mathrm{b}}^{(2)}\left(\mathbb{R}^{\nu}\right)$ пространство ограниченных дважды непрерывно дифференцируемых функций на $\mathbb{R}^{\nu}$ с нормой

$$
\|f\|_{2}=\sup _{x \in \mathbb{R}^{\nu}} \max \left\{|f(x)|,\left|\frac{\partial f}{\partial x_{i}}(x)\right|,\left|\frac{\partial^{2} f}{\partial x_{i} \partial x_{j}}(x)\right|\right\} .
$$

Введем величину

$$
\mu_{T}(f ; \eta)=\sum_{y \in \mathbb{Z}^{\nu}} \operatorname{Pr}\left(X_{T}=y ; \eta\right) f\left(\frac{y-b T}{\sqrt{T}}\right), \quad f \in C_{\mathrm{b}}^{(2)}\left(\mathbb{R}^{\nu}\right)
$$

где вектор $b$ определен в (1.1.2). Мы покажем, что

$$
\mu_{T}(f ; \eta) \rightarrow \int f(u) g(u) d u \quad \text { при } \quad T \rightarrow \infty
$$

в метрике $L_{2}(\Omega, \rho)$. Из (1.2.8) будет затем выведена наша теорема. Запишем $\mu_{T}(f ; \eta)$ в виде

$$
\mu_{T}(f ; \eta)=\mu_{T}^{0}(f)+\frac{1}{\sqrt{T}} \mathbf{M}_{T}\left(F_{T}^{f} ; \eta\right)
$$

где

$$
\begin{aligned}
\mu_{T}^{0}(f) & =\left\langle\mu_{T}(f ; \eta)\right\rangle_{\eta}=\sum_{y \in \mathbb{Z}^{\nu}} P_{0}^{T}(y) f\left(\frac{y-b t}{\sqrt{T}}\right), \\
F_{T}^{f}(y, t ; s) & =\sum_{z \in \mathbb{Z}^{\nu}} h^{T-t}(z ; s) \sqrt{T} f\left(\frac{y+z-b T}{\sqrt{T}}\right) \\
& =\sum_{u, v \in \mathbb{Z}^{\nu}} c(u ; s) P_{0}^{T-t-1}(v) \sqrt{T} f\left(\frac{y+u+v-b T}{\sqrt{T}}\right),
\end{aligned}
$$

a

$$
\mathbf{M}_{T}\left(F_{T}^{f} ; \eta\right)=\sum_{t=0}^{T-1} \sum_{y \in \mathbb{Z}^{\nu}} \mathscr{M}(y, t ; \eta) F_{T}^{f}(y, t ; \eta(y, t))
$$

Так как для распределения $P_{0}^{T}$ верна центральная предельная теорема с вектором "сноса" $b$ и матрицей $A$ (см. (1.1.2)), то

$$
\mu_{T}^{0}(f) \rightarrow \int f(u) g(u) d u \quad \text { при } \quad T \rightarrow \infty
$$

для любой функции $f \in C_{\mathrm{b}}^{(2)}\left(\mathbb{R}^{\nu}\right)$. Таким образом, остается доказать, что

$$
T^{-1}\left\langle\mathbf{M}_{T}^{2}\left(F_{T}^{f} ; \eta\right)\right\rangle_{\eta} \rightarrow 0 \quad \text { при } \quad T \rightarrow \infty .
$$

Разлагая $f((y+v+u-b T) / \sqrt{T})$ по формуле Тейлора относительно переменной $u / \sqrt{T}$ :

$$
f\left(\frac{y+v+u-b T}{\sqrt{T}}\right)=f\left(\frac{y+v-b T}{\sqrt{T}}\right)+\frac{u}{\sqrt{T}}(\nabla f)\left(\frac{y+v-b T}{\sqrt{T}}\right)+\frac{1}{T}(\mathscr{B} u, u),
$$


где $\mathscr{B}$ - матрица вторых производных $f$ в некоторой промежуточной точке, и учитывая условие $(0.5 \mathrm{a})$, получим, что

$$
F_{T}^{f}(y, t ; s)=\sum_{u \in \mathbb{Z}^{\nu}} P_{0}^{T-t-1}(v) d(s) \cdot(\nabla f)\left(\frac{y+v-b T}{\sqrt{T}}\right)+\frac{1}{\sqrt{T}} \mathscr{D}_{T}^{f}(y, t ; s),
$$

где

$$
\left|\mathscr{D}_{T}^{f}(y, t ; s)\right|<\mathrm{const}\|f\|_{2}
$$

равномерно по $y, t$ и $s$, а вектор

$$
d(s)=\sum_{u \in \mathbb{Z}^{\nu}} c(u ; s) u \in \mathbb{R}^{\nu} .
$$

Таким образом,

$$
\left\langle\left(F_{T}^{f}(y, t ; \cdot)\right)^{2}\right\rangle_{\eta}<\text { const }\|f\|_{2}^{2} \quad \text { при всех } y, t, T
$$

и, кроме того, в силу $(0.5 \mathrm{~b})$

$$
\left\langle F_{T}^{f}(y, t ; \cdot)\right\rangle_{\eta}=0 .
$$

Далее, из последнего равенства, а также того обстоятельства, что $\mathscr{M}(t, y ; \eta)$ зависит от поля в точках $\left(x^{\prime}, t^{\prime}\right)$ с $t^{\prime}<t$, а $F_{T}^{f}(y, t ; \eta(y, t))$ зависит от поля в точке $(y, t)$, легко вывести с помощью асимптотики (1.2.5), что

$$
\begin{aligned}
\left\langle\mathbf{M}_{T}^{2}\left(F_{T}^{f} ; \eta\right)\right\rangle_{\eta} & =\sum_{t=0}^{T-1} \sum_{y \in \mathbb{Z}^{\nu}}\left\langle\mathscr{M}^{2}(y, t ; \eta)\right\rangle_{\eta}\left\langle F_{T}^{2}(t, y ; \eta(t, y))\right\rangle_{\eta} \\
& <\text { const }\|f\|_{2}^{2} \sum_{t=0}^{T-1} \frac{1}{t^{\nu / 2}+1}<\text { const }\|f\|_{2}^{2} \begin{cases}T^{1 / 2}, & \nu=1, \\
\log T, & \nu=2, \\
1, & \nu \geqslant 3 .\end{cases}
\end{aligned}
$$

Отсюда и следует (1.2.14) для любой функции $f \in C_{\mathrm{b}}^{(2)}\left(\mathbb{R}^{\nu}\right)$. Выведем теперь окончательный результат (1.1.5). Для любого $\varepsilon>0$ мы можем найти две функции $f_{1}, f_{2} \in C_{\mathrm{b}}^{(2)}\left(\mathbb{R}^{\nu}\right)$ такие, что

$$
f_{1}(x) \leqslant \mathbb{1}_{G}(x) \leqslant f_{2}(x), \quad x \in \mathbb{R}^{\nu},
$$

и

$$
0<\mu_{\infty}^{0}\left(f_{2}\right)-\mu_{\infty}^{0}\left(f_{1}\right)<\varepsilon
$$

где $\mathbb{1}_{G}$ - характеристическая функция области $G$ и $\mu_{\infty}^{0}(f)=\int f(u) g(u) d u$. Обозначим

$$
\Delta_{T}(f ; \eta)=\mu_{T}(f ; \eta)-\mu_{\infty}^{0}(f)
$$

и заметим, что при любых $T$ и $\eta \in \Omega$

$$
\left|\Delta_{T}\left(\mathbb{1}_{G} ; \eta\right)\right|<\max \left\{\left|\Delta_{T}\left(f_{1} ; \eta\right)\right|,\left|\Delta_{T}\left(f_{2} ; \eta\right)\right|\right\}+\varepsilon .
$$

Отсюда

$$
\left\langle\Delta_{T}^{2}\left(\mathbb{1}_{G} ; \eta\right)\right\rangle_{\eta}<2\left(\max \left\{\left\langle\Delta_{T}^{2}\left(f_{1} ; \eta\right)\right\rangle_{\eta},\left\langle\Delta_{T}^{2}\left(f_{2} ; \eta\right)\right\rangle_{\eta}\right\}+\varepsilon^{2}\right) .
$$

Так как $\left\langle\Delta_{T}^{2}\left(f_{i} ; \eta\right)\right\rangle_{\eta} \rightarrow 0$ при $T \rightarrow \infty, i=1,2$, и $\varepsilon$ произвольно, получаем, что

$$
\left\langle\Delta_{T}^{2}\left(\mathbb{1}_{G} ; \eta\right)\right\rangle_{\eta} \rightarrow 0 \quad \text { при } \quad T \rightarrow 0,
$$

что и требовалось доказать. Теорема 1.1.1 доказана. 
1.3. Доказательство леммы 1.2.1. Введем производящую функцию для вторых моментов величин $\mathscr{M}(y, t ; \eta)$ :

$$
H(\alpha, \mu)=\sum_{t=0}^{\infty} \alpha^{t} \sum_{y \in \mathbb{Z}^{\nu}} e^{i(\mu, y-b t)}\left\langle\mathscr{M}^{2}(y, t ; \eta)\right\rangle_{\eta}
$$

где $\alpha \in \mathbb{C},|\alpha|<1$ и $\mu=\left(\mu_{1}, \ldots, \mu_{\nu}\right) \in \mathbb{T}^{\nu}{ }^{3}$ Как мы увидим ниже, ряд (1.3.1) сходится. Поскольку для независимого поля выполнено равенство $\left\langle M_{B}(\eta)\right.$ $\left.\times M_{B^{\prime}}(\eta)\right\rangle_{\eta}=0$ при $B \neq B^{\prime}$, то

$$
\left\langle\mathscr{M}^{2}(y, t ; \eta)\right\rangle_{\eta}=\sum_{\substack{B: t_{f}(B)=t \\ y_{f}(B)=y}}\left\langle M_{B}^{2}(\eta)\right\rangle_{\eta}
$$

Далее, обозначая $|B|=n>0$, мы имеем

$$
\left\langle M_{B}^{2}(\eta)\right\rangle_{\eta}=\left(P_{0}^{t_{1}}\left(y_{1}\right)\right)^{2} \prod_{j=1}^{n-1}\left\langle\left(h^{t_{j+1}-t_{j}}\left(y_{j+1}-y_{j} ; \eta\left(y_{j}, t_{j}\right)\right)\right)^{2}\right\rangle_{\eta},
$$

где, напомним, $B=\left\{\left(y_{1}, t_{1}\right), \ldots,\left(y_{n}, t_{n}\right)\right\}$. Вводя обозначения $\tau_{j}=t_{j+1}-t_{j}$, $j=1, \ldots, n-1, \tau_{0}=t_{1}$ и $z_{j}=y_{j+1}-y_{j}, j=1, \ldots, n-1, z_{0}=y_{1}$, мы запишем $\left\langle\mathscr{M}^{2}(y, t ; \eta)\right\rangle_{\eta}$ в виде

$$
\begin{aligned}
& \left\langle\mathscr{M}^{2}(y, t ; \eta)\right\rangle_{\eta} \\
& \quad=\sum_{n=1}^{t} \sum_{\tau_{0}+\tau_{1}+\cdots+\tau_{n-1}=t} \sum_{z_{0}+z_{1}+\cdots+z_{n-1}=y}\left(P_{0}^{\tau_{0}}\left(z_{0}\right)\right)^{2} \prod_{j=1}^{n-1}\left\langle\left(h^{\tau_{j}}\left(z_{j} ; \cdot\right)\right)^{2}\right\rangle_{\eta}
\end{aligned}
$$

(при $n=1$ произведение в этой формуле заменяем на единицу). Далее введем преобразование Фурье

$$
a_{t}(\mu):=\sum_{z \in \mathbb{Z}^{\nu}}\left(P_{0}^{t}(z)\right)^{2} e^{i(\mu, z-b t)}
$$

и

$$
b_{t}(\mu):=\sum_{z \in \mathbb{Z}^{\nu}}\left\langle\left(h^{t}(z ; \cdot)\right)^{2}\right\rangle_{\eta} e^{i(\mu, z-b t)}
$$

Представив

$$
\begin{aligned}
P_{0}^{t}(z) & =\int_{\mathbb{T}^{\nu}} \tilde{p}_{0}^{t}(\lambda) e^{-i(z, \lambda)} d m(\lambda), \\
h^{t}(z ; \cdot) & =\int_{\mathbb{T}^{\nu}} \tilde{p}_{0}^{t}(\lambda) \tilde{c}(\lambda ; \cdot) e^{-i(z ; \lambda)} d m(\lambda),
\end{aligned}
$$

\footnotetext{
${ }^{3}$ Поскольку экспоненту $e^{i(\mu, b) t}=\prod_{j=1}^{\nu} e^{i \mu_{j} b_{j} t}\left(\mu_{j}\right.$ и $b_{j}-$ координаты $\mu \in \mathbb{T}^{\nu}$ и $b \in \mathbb{R}^{\nu}$ соответственно) нельзя при нецелых $b_{j}$ однозначно и непрерывно определить при всех $\mu \in \mathbb{T}^{\nu}$, мы положим, что $H(\alpha, \mu)$ имеет разрывы на гиперплоскостях $\left\{\mu: \mu_{j}=\pi\right\}, j=1, \ldots, \nu$. Таким образом, $H(\alpha, \mu)$ является гладкой функцией в окрестности точки $\mu=0$ на торе $\mathbb{T}^{\nu}=(-\pi, \pi)^{\nu}$. Это замечание относится и к другим вводимым ниже функциям от $\mu$.
} 
где $\tilde{c}(\lambda ; \cdot)=\sum_{u \in \mathbb{Z}^{\nu}} c(u ; \cdot) e^{i(u, \lambda)}$ и, напомним, $d m(\lambda)=\frac{d^{\nu} \lambda}{(2 \pi)^{\nu}}-$ нормированная мера Хаара на торе $\mathbb{T}^{\nu}$, мы получим

$$
\begin{aligned}
a_{t}(\mu) & =\int d m\left(\lambda_{1}\right) d m\left(\lambda_{2}\right)\left(\tilde{p}_{0}\left(\lambda_{1}\right) \tilde{p}_{0}\left(\lambda_{2}\right)\right)^{t} \sum_{z \in \mathbb{Z}^{\nu}} e^{-i\left(\lambda_{1}+\lambda_{2}, z\right)} e^{i(\mu, z-b t)} \\
& =e^{-i t(\mu, b)} \int_{\mathbb{T}^{\nu}} d m(\lambda)\left(\tilde{p}_{0}(\lambda) \tilde{p}_{0}(\mu-\lambda)\right)^{t} \\
& =\int d m(\lambda)(\hat{p}(\lambda) \hat{p}(\mu-\lambda))^{t},
\end{aligned}
$$

где $\hat{p}(\lambda)=e^{-i(\lambda, b)} \tilde{p}_{0}(\lambda)$. Подобным же образом получим, что

$$
b_{t}(\mu)=\int d m(\lambda) \hat{g}(\lambda, \mu-\lambda)(\hat{p}(\lambda) \hat{p}(\mu-\lambda))^{t-1},
$$

где $\hat{g}\left(\lambda_{1}, \lambda_{2}\right)=e^{-i\left(\lambda_{1}+\lambda_{2}, b\right)}\left\langle\tilde{c}\left(\lambda_{1} ; \cdot\right) \tilde{c}\left(\lambda_{2} ; \cdot\right)\right\rangle$. Подставляя разложение (1.3.4) в формулу (1.3.1), мы найдем, что

$$
\begin{aligned}
H(\alpha, \mu) & =\sum_{t=1}^{\infty} \alpha^{t} \sum_{n=1}^{t} \sum_{\tau_{0}+\tau_{1}+\cdots+\tau_{n-1}=t} a_{\tau_{0}}(\mu) \prod_{j=1}^{n-1} b_{\tau_{j}}(\mu) \\
& =\sum_{\tau_{0}=0}^{\infty} \alpha^{\tau_{0}} a_{\tau_{0}}(\mu) \sum_{n=1}^{\infty}\left(\sum_{\tau=1}^{\infty} \alpha^{\tau} b_{\tau}(\mu)\right)^{n-1} .
\end{aligned}
$$

Таким образом, из (1.3.7) и (1.3.8) мы видим, что

$$
\begin{aligned}
N(\alpha, \mu) & :=\sum_{t=0}^{\infty} \alpha^{t} a_{t}(\mu)=\int_{\mathbb{T}^{\nu}} \frac{d m(\lambda)}{1-\alpha \hat{p}(\lambda) \hat{p}(\mu-\lambda)}, \\
M(\alpha, \mu) & :=\sum_{t=1}^{\infty} \alpha^{t} b_{t}(\mu)=\int_{\mathbb{T}^{\nu}} \frac{\hat{g}(\lambda, \mu-\lambda) d m(\lambda)}{1-\alpha \hat{p}(\lambda) \hat{p}(\mu-\lambda)} .
\end{aligned}
$$

В этих обозначениях производящая функция $H(\alpha, \mu)$ имеет вид

$$
H(\alpha, \mu)=\frac{N(\alpha, \mu)}{1-M(\alpha, \mu)} .
$$

Обозначим ради краткости через $q_{t}$ левую часть $(1.2 .5)$, т. е.

$$
q_{t}=\sum_{y \in \mathbb{Z}^{\nu}}\left\langle\mathscr{M}^{2}(y, t ; \eta)\right\rangle_{\eta} .
$$

Из (1.3.1) мы находим, что

$$
\sum \alpha^{t} q_{t}=H_{0}(\alpha):=H(\alpha, 0)
$$

Вводя аналогичные обозначения $N(\alpha, 0)=: N_{0}(\alpha)$ и $M(\alpha, 0)=: M_{0}(\alpha)$, мы запишем (1.3.11) в виде

$$
H_{0}(\alpha)=\frac{N_{0}(\alpha)}{1-M_{0}(\alpha)} .
$$


Обозначим через $\Pi \subset \mathbb{C}$ комплексную плоскость с разрезом вдоль интервала $(1, q)$ на вещественной оси, где

$$
q=\sup _{\lambda} \frac{1}{\left|\tilde{p}_{0}^{2}(\lambda)\right|} \leqslant \infty
$$

ПРЕДЛОЖЕНИЕ 1.3.1. Функции $N_{0}(\alpha)$ и $M_{0}(\alpha)$ аналитичны по $\alpha \in \Pi$. Кроме того, $M_{0}(\alpha) \neq 1$ при $\alpha \in \Pi$.

ДоказАтельство. Так как $\hat{p}(\lambda) \hat{p}(-\lambda)=\left|\tilde{p}_{0}(\lambda)\right|^{2} \leqslant 1$, то $1-\alpha\left|\tilde{p}_{0}(\lambda)\right|^{2} \neq 0$ при $\alpha \in$ П. Отсюда и из формул (1.3.10) немедленно вытекает аналитичность функций $N_{0}(\alpha)$ и $M_{0}(\alpha)$. Что касается второго утверждения, то заметим, что при малых $\lambda$

$$
0<1-\left|\tilde{p}_{0}(\lambda)\right|^{2}=2(\lambda, C \lambda)+o\left(\lambda^{2}\right),
$$

где $C$ - положительно-определенная матрица вторых производных $\tilde{p}_{0}(\lambda)$ при $\lambda=0$ (условие iii)). Функция $\hat{g}(\lambda,-\lambda)$, равная $\left\langle|\tilde{c}(\lambda ; \cdot)|^{2}\right\rangle$, имеет порядок $O\left(\lambda^{2}\right)$. Таким образом,

$$
\lim _{\alpha \rightarrow 1-0} M_{0}(\alpha)=M_{0}(1)=\int_{\mathbb{T}^{\nu}} \frac{\left\langle|\tilde{c}(\lambda ; \cdot)|^{2}\right\rangle_{\eta}}{1-\left|\tilde{p}_{0}(\lambda)\right|^{2}} d m(\lambda)<\infty \quad \text { и } \quad M_{0}(1)>0 .
$$

С другой стороны,

$$
\begin{aligned}
1-M_{0}(1) & =\int_{\mathbb{T}^{\nu}} \frac{1-\left|\tilde{p}_{0}\right|^{2}-\left\langle|\tilde{c}(\lambda ; \cdot)|^{2}\right\rangle_{\eta}}{1-\left|\tilde{p}_{0}(\lambda)\right|^{2}} d m(\lambda) \\
& =\int_{\mathbb{T}^{\nu}} \frac{1-\left\langle\left|\tilde{p}_{0}(\lambda)+\tilde{c}(\lambda ; \cdot)\right|^{2}\right\rangle_{\eta}}{1-\left|\tilde{p}_{0}(\lambda)\right|^{2}} d m(\lambda)>0 .
\end{aligned}
$$

Последнее неравенство следует из условия iv), утверждающего, что

$$
\left\langle\left|\tilde{p}_{0}(\lambda)+\tilde{c}(\lambda ; \cdot)\right|^{2}\right\rangle_{\eta}<1
$$

на множестве значений $\lambda$ положительной меры $m(\lambda)$ (и что при всех $\lambda$ левая часть (1.3.13) не превосходит единицы). Таким образом, $M_{0}(1)<1$. Нетрудно проверить, что функция $M_{0}(\alpha)$ монотонно возрастает на полуоси $(-\infty, 1)$ и, таким образом, $M_{0}(\alpha)<1$ при всех $\alpha$ из этого промежутка. Представим $M_{0}(\alpha)$ в виде

$$
M_{0}(\alpha)=\frac{1}{\alpha} \int \frac{\left\langle|\tilde{c}(\lambda ; \cdot)|^{2}\right\rangle_{\eta}}{1 / \alpha-\left|\tilde{p}_{0}(\lambda)\right|^{2}} d m(\lambda)
$$

откуда видно, что $M_{0}(\alpha)<0$ при $\alpha>q$ (если $\left.q<\infty\right)$ и что $\operatorname{Im} M_{0}(\alpha) \neq 0$ при $\operatorname{Im} \alpha \neq 0$. Предложение 1.3.1 доказано.

Как следует из этого предложения, функция $H_{0}(\alpha)$ также аналитична в П. В следующей лемме описаны особенности этой функции в окрестности точки $\alpha=1$.

Лемма 1.3.1. Для любого $\nu=1,2, \ldots$ существует комплексная окрестность $U$ точки $\alpha=1$ такая, что для $\alpha \in U$ функиия $H_{0}(\alpha)$ представляется в виде

$$
H_{0}(\alpha)=H_{\nu}^{\sharp}(\alpha)+(1-\alpha)^{s-1 / 2} h_{\nu}^{\sharp}(\alpha)
$$


для нечетных $\nu=2 s+1 u$

$$
H_{0}(\alpha)=H_{\nu}^{\sharp}(\alpha)+(1-\alpha)^{s} \log (1-\alpha) h_{\nu}^{\sharp}(\alpha)+R_{\nu}(\alpha)
$$

для четных $\nu=2 s+2 ;$ при этом

$$
\left|R_{\nu}(\alpha)\right|<\text { const }|1-\alpha|^{s+1}|\log (1-\alpha)|^{2} .
$$

Функции $H_{\nu}^{\sharp}(\alpha)$ и $h_{\nu}^{\sharp}(\alpha)$ аналитичны в $U$, причем значение $h_{\nu}^{\sharp}(1)$ не равно нулю и вещественно. $B$ бормуле $(1.3 .14 \mathrm{a})$ под $(1-\alpha)^{1 / 2}$ понимается ветвь этой функции, которая положительна при вещественных $\alpha<1$, а в формулах (1.3.14b) и (1.3.14c) $\log (1-\alpha)$ полагается вещественным при $\alpha<1$.

Доказательство этой технической леммы приведено в приложении. Здесь мы выведем из нее лемму 1.2.1.

ЗАВЕРШЕНИЕ ДОКАЗАТЕЛЬСТВА ЛЕММЫ 1.2.1. АсимПтотика величин $q_{t}$, являющихся коэффициентами ряда Тейлора функции $H_{0}(\alpha)$ в точке $\alpha=0$, может быть найдена с помощью их представления в виде интегралов

$$
q_{t}=\frac{1}{2 \pi i} \int_{C} \frac{H_{0}(\alpha)}{\alpha^{t+1}} d \alpha
$$

по любому контуру $C \subset \Pi$, обходящему точку $\alpha=0$ против часовой стрелки и не содержащему внутри себя особенностей функции $H_{0}(\alpha)$. В качестве такого контура выберем кривую, состоящую из двух частей: окружность $\Gamma_{\delta}$ с центром в нуле и радиусом $R=1+\delta / 2, \delta>0$, и с выколотой точкой $\bar{\alpha}=1+\delta / 2$, лежащей на разрезе $[1, q]$, а к окружности мы присоединим петлю $\gamma$, начинающуюся и кончающуюся в точке $\bar{\alpha}$ и обходящую часть разреза $(1, \bar{\alpha})$. При этом число $\delta$ и петлю $\gamma$ мы выберем так, чтобы $\gamma$ лежала в окрестности $U$ точки $\alpha=1$, для которой справедливы представления (1.3.14a), (1.3.14b) функции $H_{0}(\alpha)$ из леммы 1.3.1. Этот контур изображен на рис. 2.

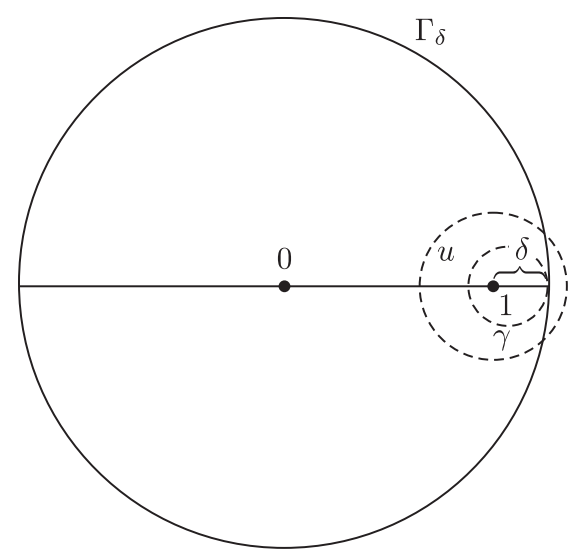

Рис. 2. Контур интегрирования $C$ : окружность $\Gamma_{\delta}$ и петля $\gamma$

Забегая вперед, отметим, что в дальнейшем иногда окажется удобным стянуть петлю $\gamma$ к разрезу и считать, что $\gamma$ - это обход по верхнему (от $\bar{\alpha}$ до 1 ), а затем по нижнему (от 1 до $\bar{\alpha}$ ) берегам разреза. Заметим, что, поскольку 
функция $H_{0}(\alpha)$ ограничена на окружности $\Gamma_{\delta}$, вклад в интеграл (1.3.15) от интегрирования по $\Gamma_{\delta}$ не превосходит

$$
\text { const } \frac{1}{(1+\delta / 2)^{t}}
$$

т. е. убывает экспоненциально с ростом $t$. Таким образом, главная (“степенная") часть асимптотики интеграла (1.3.15) создается интегрированием по петле $\gamma$. Мы рассмотрим по отдельности случай четных и нечетных $\nu$.

А. Случай нечетной размерности $\nu=2 s+1$. Очевидно, что аналитическая часть $H^{\sharp}$ в разложении (1.3.14a) дает нулевой вклад в интеграл

$$
\frac{1}{2 \pi i} \int_{\gamma} \frac{H_{0}(\alpha)}{\alpha^{t+1}} d \alpha
$$

а сингулярная часть в (1.3.14a) содержит интегрируемую в точке $\alpha=1$ особенность при всех $s \geqslant 0$ и мы можем, как говорилось выше, стянуть петлю к обходу по обоим берегам разреза, т. е.

$$
\frac{1}{2 \pi i} \int_{\gamma} \frac{H_{0}(\alpha)}{\alpha^{t+1}} d \alpha=-\frac{1}{2 \pi i} \int_{1}^{\bar{\alpha}=1+\delta / 2} \frac{\Delta H_{0}(\alpha)}{\alpha^{t+1}} d \alpha,
$$

где $\Delta H_{0}(\alpha)$ - скачок функции $H_{0}(\alpha)$ при переходе с верхнего берега разреза на нижний. Нетрудно подсчитать, что

$$
\begin{aligned}
\Delta H_{0}(\alpha) & =h_{\nu}^{\sharp}(\alpha)(\alpha-1)^{s-1 / 2}\left[e^{+i \pi(s-1 / 2)}-e^{-i \pi(s-1 / 2)}\right] \\
& =(-1)^{s}(-2 i) h_{\nu}^{\sharp}(1+u) u^{s-1 / 2},
\end{aligned}
$$

где мы положим $\alpha=1+u$. Итак, интеграл (1.3.16) равен

$$
\begin{aligned}
& -\frac{(-1)^{s}}{\pi} \int_{0}^{\delta / 2} \frac{u^{s-1 / 2} h_{\nu}^{\sharp}(1+u)}{(1+u)^{t+1}} d u \\
& \quad=-\frac{(-1)^{s}}{\pi(t+1)^{s / 2+1}} \int_{0}^{\delta(t+1) / 2} \frac{z^{s-1 / 2} h_{\nu}^{\sharp}\left(1+\frac{z}{t+1}\right)}{\left(1+\frac{z}{t+1}\right)^{t+1}} d z,
\end{aligned}
$$

где произведена замена $u=\frac{z}{t+1}$.

Интеграл в правой части (1.3.17) сходится при $t \rightarrow \infty$ к величине

$$
h_{\nu}^{\sharp}(1) \int_{0}^{\infty} z^{s-1 / 2} e^{-z} d z=h_{\nu}^{\sharp}(1) \Gamma(s+1 / 2) .
$$

Так как $q_{t}>0$ при всех $t$, то

$$
0<-(-1)^{s} h_{\nu}^{\sharp}(1)=\left|h_{\nu}^{\sharp}(1)\right|
$$

и мы получаем окончательную асимптотику для $q_{t}$ в случае нечетной размерности:

$$
q_{t}=\frac{\left|h_{\nu}^{\sharp}(1)\right|}{\pi} \frac{\Gamma(\nu / 2)}{(t+1)^{\nu / 2}}(1+o(1)) .
$$


В. Случай четной размерности $\nu=2 s+2$. Повторяя предыдущие рассуждения, получим, что главная часть интеграла (1.3.15) представляется в виде

$$
-\frac{1}{2 \pi i} \int_{1}^{1+\delta / 2} \frac{\Delta H_{0}(\alpha)}{\alpha^{t+1}} d \alpha=\frac{(-1)^{s}}{\pi} \int_{0}^{\delta / 2} \frac{u^{s} h_{\nu}^{\sharp}(1+u)}{(1+u)^{t+1}} d u+\int_{0}^{\delta / 2} \frac{\Delta R(1+u)}{(1+u)^{t+1}} d u .
$$

Первый интеграл в правой части (1.3.19) после замены $u=\frac{z}{t+1}$ приводит к асимптотике

$$
\frac{(-1)^{s} h_{\nu}^{\sharp}(1)}{(t+1)^{s+1}} \Gamma(\nu / 2)(1+o(1)) .
$$

Для второго интеграла получаем оценку (см. (1.3.14c))

$$
\begin{aligned}
&\left|\int_{0}^{\delta / 2} \frac{\Delta R(1+u)}{(1+u)^{t+1}} d u\right|<\mathrm{const} \int_{0}^{\delta / 2} \frac{u^{s+1}\left(\log ^{2} u+\pi^{2}\right)}{(1+u)^{t+1}} d u \\
&=O\left(\frac{1}{t^{s+2}}\right)+O\left(\frac{\log ^{2}(t+1)}{t^{s+2}}\right)=o\left(\frac{1}{t^{s+1}}\right) .
\end{aligned}
$$

Итак, во всех случаях мы находим, что

$$
q_{t}=\sum_{y \in \mathbb{Z}^{\nu}}\left\langle\mathscr{M}^{2}(y, t ; \eta)\right\rangle=\frac{\left|h_{\nu}^{\sharp}(1)\right|}{\pi} \Gamma(\nu / 2) \frac{1}{(t+1)^{\nu / 2}}(1+o(1)) .
$$

Лемма 1.2.1 доказана.

\section{4. Дополнительные вопросы.}

1.4.1. Другие подходы. Основной результат - центральная предельная теорема - в случае, когда взаимодействие частицы со средой мало (т. е. мала функция $c(u ; s)$ в $(0.4))$, может быть получен и другими методами (см. [8], [7], [9]). Главный прием в работе [8] состоит в тонкой комбинаторной оценке моментов

$$
\left\langle\left(\mathscr{M}_{T, T^{\prime}}(\eta)\right)^{2 n}\right\rangle_{\eta}
$$

при всех $n$ и $T<T^{\prime}$ для величины $\mathscr{M}_{T, T^{\prime}}(\eta)$ общего вида

$$
\mathscr{M}_{T, T^{\prime}}(\eta)=\sum_{t=T}^{T^{\prime}-1} \sum_{y \in \mathbb{Z}^{\nu}} \mathscr{M}(y, t ; \eta) G(y, t ; \eta(y, t))
$$

где $\mathscr{M}(y, t ; \eta)$ определена формулой $(1.2 .3 \mathrm{a})$, а $G(y, t ; s)$ - функция из некоторого общего класса функций на $\mathbb{Z}^{\nu} \times \mathbb{Z}^{1} \times S$. В этой же статье кроме главного неслучайного гауссовского члена в асимптотике для $\mu_{T}(f ; \eta)($ см. $(1.2 .7))$ получен - в случае размерностей $\nu \geqslant 3-$ и следующий ее член, зависящий от поля $\eta$ :

$$
\mu_{T}(f ; \eta)=\mu_{\infty}^{0}(f)+\frac{1}{\sqrt{T}} \Phi(f ; \eta)(1+o(1))
$$

где

$$
\Phi(f ; \eta)=\int_{\mathbb{R}^{\nu}} g(u)(\varepsilon(\eta) \cdot(\nabla f)(u)) d u, \quad f \in C_{\mathrm{b}}^{(2)}\left(\mathbb{R}^{\nu}\right)
$$


Вектор $\varepsilon(\eta)$ определяется как предельная поправка в асимптотике вектора среднего сноса:

$$
\varepsilon(\eta)=\lim _{T \rightarrow \infty} \varepsilon_{T}(\eta)
$$

а $\varepsilon_{T}(\eta)$ задается формулой

$$
\varepsilon_{T}(\eta)=\sum_{u \in \mathbb{Z}^{\nu}} u \operatorname{Pr}\left(X_{T}=u \mid X_{0}=0 ; \eta\right)-b T=\bar{X}_{T}(\eta)-b T,
$$

где черта означает среднее по распределению $\operatorname{Pr}\left(X_{T} \mid X_{0}=0 ; \eta\right)$. Существование предела (1.4.4a) также установлено в статье [8], равно как и существование пределов для поправок к ковариации вектора $X_{T}$ :

$$
\varphi_{j k}(\eta)=\lim _{T \rightarrow \infty}\left[\overline{\left(X_{T}-\bar{X}_{T}(\eta)\right)_{j}\left(X_{T}-\bar{X}_{T}(\eta)\right)_{k}}-c_{j k} T\right],
$$

$C=\left\{c_{j k}\right\}$ - матрица ковариаций для свободного блуждания (см. (1.1.2)). Следующий член асимптотики (1.4.2) также найден в [8] для случая размерности $\nu \geqslant 5$. К сожалению, методы обсуждаемой работы не позволяют исследовать случай $\nu=1$. Для этого в статье [9] был использован другой метод, при котором центральная предельная теорема для случая $\nu=1$ выводилась с помощью установления пределов для моментов:

$$
\lim _{T \rightarrow \infty}\left(\frac{\bar{X}_{T}-b T}{\sqrt{T}}\right)^{k}=m_{k}, \quad k=0,1,2, \ldots,
$$

где $m_{k}$ - моменты гауссовского распределения $g(u)$. Этот же прием пригоден и для любой размерности $\nu$. Отметим, что главной пружиной в доказательстве (1.4.6) явилось наблюдение, что последовательность

$$
N_{T}^{(k)}(\eta)=\sum_{t=0}^{T-1} \sum_{y \in \mathbb{Z}^{\nu}} \mathscr{M}(y, t ; \eta) \sum_{u \in \mathbb{Z}^{\nu}} c(u ; \eta(y, t))(u+y-b(t+1))^{k}, \quad T=1,2, \ldots,
$$

является мартингалом относительно $T$ и для оценки вероятности

$$
\operatorname{Pr}\left(\sup _{T_{1}<T<T_{2}}\left(N_{T}^{(k)}(\eta)\right)^{2}>T_{1}^{p}\right)
$$

можно применить неравенство Колмогорова для мартингалов (см. [6]).

1.4.2. Поправка к гауссовскому члену в асимптотике $\mu_{T}(f ; \eta)$ в одномерном случае. Как мы видели выше (см. (1.4.2)), при малом взаимодействии $c(u ; s)$ в случае размерности $\nu \geqslant 3$ разность

$$
\mu_{T}(f ; \eta)-\mu_{\infty}^{0}(f)=\Delta_{T}(f ; \eta)
$$

оказывается величиной порядка $1 / \sqrt{T}$ и, кроме того, величина $\sqrt{T} \Delta T(f ; \eta)$ имеет определенный (зависящий от поля) предел $\Phi(f ; \eta)$. В одномерном случае картина другая: разность $\Delta_{T}(f ; \eta)$ оказывается уже величиной порядка $1 / T^{1 / 4}$, причем $T^{1 / 4} \Delta_{T}(f ; \eta)$ уже не имеет предела при $T \rightarrow \infty$, а имеет лишь предельное распределение; это распределение является гауссовским со средним нуль и конечной дисперсией (явно вычисленной в работе [10]). В статье [11] показано, что в одномерном случае $\varepsilon_{T}(\eta)$ (см. выше $(1.4 .4 \mathrm{~b})$ ) имеет порядок $T^{1 / 4}$ и предельное распределение для $\varepsilon_{T}(\eta) / T^{1 / 4}$ оказывается нормальным. 
1.4.3. Локальная предельная теорема. (См. [12].) До сих пор говорилось о центральной предельной теореме в интегральной форме, т. е. о распределении вероятностей того, что величина $u_{T}=\left(X_{T}-b T\right) / \sqrt{T}$ заключена в ограниченной области $G \subset \mathbb{R}^{\nu}$. Локальная предельная теорема представляет собой асимптотику вероятности тех или иных значений величины $u_{T}$ при условии, что $\left|u_{T}\right|=o\left(T^{1 / 6}\right)$.

Прежде чем привести точную формулировку локальной предельной теоремы для блуждания в случайной среде, введем следующее понятие: функционал $\Phi(\eta)$ от поля $\eta$ (определенный, быть может, только на подмножестве $\widetilde{\Omega} \subset \Omega$ конфигураций поля полной меры) называется квазилокализованным в начале координат в $\mathbb{Z}^{\nu+1}$, если

$$
\sup _{\eta_{1}\left|O_{R}=\eta_{2}\right| O_{R}}\left|\Phi\left(\eta_{1}\right)-\Phi\left(\eta_{2}\right)\right| \rightarrow 0 \quad \text { при } \quad R \rightarrow \infty .
$$

Здесь $O_{R} \subset \mathbb{Z}^{\nu+1}$ - куб со стороной $2 R$ и центром в нуле, а верхняя грань берется по всем парам конфигураций $\eta_{1}, \eta_{2} \in \widetilde{\Omega}$, совпадающим внутри куба $O_{R}$. Наглядно (1.4.7) означает, что $\Phi(\eta)$ существенно зависит от переменных поля $\eta(x, t)$ в точках $(x, t)$, близких к началу координат.

Далее удобно отсчитывать координаты от точки $\left(X_{T}, T\right) \in \mathbb{Z}^{\nu+1}$, т. е. положить

$$
\eta^{\left(X_{T}, T\right)}(y, t)=\eta\left(X_{T}-y, T-t\right) .
$$

Итак, локальная предельная теорема для блуждания в случайной среде: $n p u$ выполнении всех предположений, принятых в теореме 1.1.1, существует такой квазилокализованный в начале координат функционал от поля $\Phi(\eta)$, что

$$
\operatorname{Pr}\left(X_{T}=\bar{x}_{T} ; \eta\right)=g\left(\frac{\bar{x}_{T}-b T}{\sqrt{T}}\right) \frac{1}{T^{\nu / 2}}\left(\Phi\left(\eta^{\left(\bar{x}_{T}, T\right)}\right)+o(1)\right), \quad T \rightarrow \infty,
$$

при условии, что последовательность $\bar{x}_{T}$ удовлетворяет условию

$$
\left|\bar{x}_{T}-b T\right|=o\left(T^{1 / 6}\right), \quad T \rightarrow \infty ;
$$

при этом $\langle\Phi(\eta)\rangle=1$.

\section{§ 2. Блуждание в марковской среде; annealed-модель}

2.1. Центральная предельная теорема для положения частицы. Мы изложим здесь центральную предельную теорему для случая annealed-модели, описанной во введении. Напомним, что в этой модели положение блуждающей частицы $X_{t} \in \mathbb{Z}^{\nu}$ и значение поля $\eta_{t}=\left\{\eta_{t}(x), x \in \mathbb{Z}^{\nu}\right\}$ в один и тот же момент времени образуют марковскую цепь, причем переходные вероятности этой цепи определяются правилами 1)-3) из введения (см. с. 30).

Для того чтобы избежать громоздкости в изложении, мы введем следующие упрощения:

a) поле $\eta$ принимает только два значения: $S=\{-1,1\}$;

b) семейство матриц $Q_{u}$ в формуле $(0.11)$ стабилизируется при $|u| \geqslant 1$; иными словами,

$$
\operatorname{Pr}\left(\eta_{t+1}(z)=s \mid X_{t}=\bar{x}, \eta_{t}=\bar{\eta}\right)=\left\{\begin{array}{lll}
\hat{q}(\bar{\eta}(z), s) & \text { при } \quad z \neq \bar{x} \\
q_{0}(\bar{\eta}(z), s) & \text { при } \quad z=\bar{x}
\end{array}\right.
$$


где $\left\{\hat{q}\left(s^{\prime}, s\right)\right\}_{s^{\prime}, s \in S}=\widehat{Q}$ и $\left\{q_{0}\left(s^{\prime}, s\right)\right\}_{s^{\prime}, s \in S}=Q_{0}$ - две стохастические матрицы. Мы предположим, что марковская цепь $\left\{\bar{\eta}_{t}, t \in \mathbb{Z}^{1}\right\}$ с пространством состояний $S=\{-1,1\}$ и переходными вероятностями

$$
\operatorname{Pr}\left(s^{\prime} \rightarrow s\right)=\hat{q}\left(s^{\prime}, s\right)
$$

стационарна, эргодична, и ее единственную инвариантную вероятностную меру на $S$ мы обозначим $\pi(s)$. Далее, мы примем, что возмущения, вносимые полем в эволюцию частицы и, наоборот, частицей в эволюцию поля, - малы, т. е.

$$
\varepsilon_{1}=\max _{u, s}|c(u ; s)| \ll 1 \quad \text { и } \quad \varepsilon_{2}=\max _{s, s^{\prime}}\left|\Delta\left(s^{\prime}, s\right)\right| \ll 1,
$$

где $\Delta\left(s, s^{\prime}\right)=\hat{q}\left(s, s^{\prime}\right)-q_{0}\left(s, s^{\prime}\right)$.

Итак, будет доказана следующая теорема.

ТЕорема 2.1.1 (локальная предельная теорема). Пусть выполнены все условия, касающиеся введенной annealed-модели. Тогда при произвольном начальном распределении поля в момент $t=0$ верна асимптотика

$$
\operatorname{Pr}\left(X_{T}=x \mid X_{0}=x_{0}\right)=\frac{\sqrt{\operatorname{det} A}}{(2 \pi T)^{\nu / 2}} \exp \left\{-\frac{1}{2 T} \mathscr{A}\left(x-x_{0}-b T\right)\right\}(1+o(1)),
$$

где вектор $b \in \mathbb{R}^{\nu}, a \mathscr{A}(v)$ - положительно-определенная квадратичная форма c матрищей $A=\left\{a_{i j}\right\}$ :

$$
\mathscr{A}(v)=\sum_{i, j} a_{i j} v_{i} v_{j}
$$

$v=\left(v_{1}, \ldots, v_{\nu}\right) \in \mathbb{R}^{\nu}$. Асимптотика (2.1.4) равномерна относительно всех $x$ в любой области вида

$$
\left|x-x_{0}-b T\right|<T^{1 / 2+\varepsilon}, \quad \varepsilon<\frac{1}{6} .
$$

СлЕДСтвиЕ (центральная предельная теорема в интегральной форме). Bъполнено предельное соотношение

$$
\operatorname{Pr}\left(X_{T}-X_{0} \in \sqrt{T} G+b T \mid X_{0}=x_{0}\right) \rightarrow \int_{G} g(u) d u, \quad T \rightarrow \infty,
$$

для любой ограниченной области $G \subset \mathbb{R}^{\nu}$ с кусочно гладкой гранищей.

Действительно, рассмотрим, как и выше, величину

$$
\mu_{T}(f)=\sum_{x \in \mathbb{Z}^{\nu}} \operatorname{Pr}\left(X_{T}=x \mid X_{0}=x_{0}\right) f\left(\frac{x-b T-x_{0}}{\sqrt{T}}\right),
$$

где $f$ - гладкая ограниченная финитная функция на $\mathbb{R}^{\nu}$.

Из теоремы 2.1.1 следует, что

$$
\left|\mu_{T}(f)-\sum_{x \in \mathbb{Z}^{\nu}} \frac{\sqrt{\operatorname{det} A}}{(2 \pi T)^{\nu / 2}} \exp \left\{-\frac{1}{2} \mathscr{A}\left(\frac{x-b T-x_{0}}{\sqrt{T}}\right)\right\} f\left(\frac{x-b T-x_{0}}{\sqrt{T}}\right)\right| \rightarrow 0
$$


при $T \rightarrow \infty$. С другой стороны, сумма в (2.1.8) является римановой суммой для интеграла $\int g(u) f(u) d u$ и при $T \rightarrow \infty$ имеет предел

$$
\int g(u) f(u) d u
$$

Отсюда $\mu_{T}(f) \rightarrow \int g(u) f(u) d u$. Далее мы всегда можем выбрать две гладкие ограниченные финитные функции $f_{1}$ и $f_{2}$ так, что

$$
f_{1} \leqslant \mathbb{1}_{G} \leqslant f_{2}
$$

и

$$
0<\int f_{2}(u) g(u) d u-\int f_{1}(u) g(u) d u<\varepsilon .
$$

Отсюда и следует (2.1.7).

2.2. Доказательство теоремы 2.1.1; приготовления. Обозначим через $\overline{\mathscr{T}}$ стохастический оператор марковской цепи $\left\{\bar{\eta}_{t}\right\}$ с переходными вероятностями (2.1.2). Оператор $\overline{\mathscr{T}}$ действует по формуле

$$
(\overline{\mathscr{T}} f)\left(s^{\prime}\right)=\sum_{s \in S} f(s) \hat{q}\left(s^{\prime}, s\right) .
$$

У оператора $\overline{\mathscr{T}}$ имеется два собственных вектора: $e_{0} \equiv 1$ с собственным значением $\mu_{0}=1$ и $e_{1}(s)$ с вещественным собственным значением $\mu,|\mu|<1$. При этом векторы $e_{0}$ и $e_{1}$ ортогональны относительно скалярного произведения в $l_{2}(S, \pi)$ :

$$
\left(e_{1}, e_{0}\right)_{\pi} \equiv \sum e_{1}(s) e_{0}(s) \pi(s)=\sum e_{1}(s) \pi(s)=0
$$

и нормированы.

Рассмотрим гильбертово пространство

$$
\mathscr{H}=L_{2}\left(S^{\mathbb{Z}^{\nu}}, \Pi_{0}\right) \otimes l_{2}\left(\mathbb{Z}^{\nu}\right)
$$

где $\Pi_{0}=\pi^{\mathbb{Z}^{\nu}}$, и ортонормированный базис в нем:

$$
\Psi_{\gamma, z}(\eta, x)=\prod_{y \in \gamma} e_{1}(\eta(y)) \delta_{x, z} .
$$

Здесь $\gamma \subset \mathbb{Z}^{\nu}$ - конечное подмножество решетки $\mathbb{Z}^{\nu}$, а $\delta_{x, z}$ - символ Кронекера.

Пусть $\mathscr{T}$ - стохастический оператор марковской цепи $\left\{X_{t}, \eta_{t}\right\}$ :

$$
(\mathscr{T} f)(\eta, x)=\left\langle f\left(X_{1}, \eta_{1}\right) \mid X_{0}=x, \eta_{0}=\eta\right\rangle \text {, }
$$

где $\left\langle\cdot \mid X_{0}=x, \eta_{0}=\eta\right\rangle-$ условное среднее по распределению значений цепи в момент $t=1$ при условии, что фиксированы ее значения при $t=0$. Очевидно, что

$$
\operatorname{Pr}\left(X_{1}=x \mid X_{0}=x_{0}\right)=\int\left(\mathscr{T} \Psi_{\varnothing, x}\right)\left(\eta, x_{0}\right) d \Pi(\eta),
$$


где $\Pi$ - начальное распределение поля $\left\{\eta_{t}\right\}$ при $t=0$. Отсюда легко следует, что при любом $T>0$ вероятность $\operatorname{Pr}\left(X_{T}=x \mid X_{0}=x_{0}\right)$ можно представить в виде

$$
\operatorname{Pr}\left(X_{T}=x \mid X_{0}=x_{0}\right)=\int\left(\mathscr{T}^{T} \Psi_{\varnothing, x}\right)\left(\eta, x_{0}\right) d \Pi(\eta) .
$$

Формула (2.2.5) подсказывает, что асимптотика этой вероятности при больших $T$ может быть найдена, если известна спектральная структура оператора $\mathscr{T}$ вблизи правого конца его спектра. Изучению этой структуры и будет посвящена остальная часть доказательства.

Обозначим $R_{\gamma, z ; \gamma^{\prime}, z^{\prime}}$ матричные элементы $\mathscr{T}$ в базисе $\left\{\Psi_{\gamma, z}\right\}$ :

$$
R_{\gamma, z ; \gamma^{\prime}, z^{\prime}}=\left(\mathscr{T} \Psi_{\gamma, z}, \Psi_{\gamma^{\prime}, z^{\prime}}\right) \mathscr{H} .
$$

Несложный подсчет приводит к выражению

$$
\begin{aligned}
R_{\gamma, z ; \gamma^{\prime}, z^{\prime}}=p_{0} & \left(z-z^{\prime}\right) \mu^{|\gamma|-1}\left[\mu \delta_{\gamma, \gamma^{\prime}}+\mathbb{1}_{\left\{z^{\prime} \in \gamma\right\}} \delta_{\gamma, \gamma^{\prime}} \cdot \beta+\mathbb{1}_{\left\{z^{\prime} \in \gamma\right\}} \delta_{\gamma \backslash\left\{z^{\prime}\right\}, \gamma^{\prime}} \cdot \varphi\right] \\
& +c_{1}\left(z-z^{\prime}\right) \mu^{|\gamma|-1}\left[\left(1-\mathbb{1}_{\left\{z^{\prime} \in \gamma\right\}}\right) \mu \cdot \delta_{\gamma \cup\left\{z^{\prime}\right\}, \gamma^{\prime}}+\mathbb{1}_{\left\{z^{\prime} \in \gamma\right\}} \mu \alpha \cdot \delta_{\gamma, \gamma^{\prime}}\right. \\
& +\mathbb{1}_{\left\{z^{\prime} \in \gamma\right\}} \mu \cdot \delta_{\gamma \backslash\left\{z^{\prime}\right\}, \gamma^{\prime}}+\mathbb{1}_{\left\{z^{\prime} \in \gamma\right\}} \delta_{\gamma, \gamma^{\prime}} \cdot(\varphi+\alpha \beta) \\
& \left.+\mathbb{1}_{\left\{z^{\prime} \in \gamma\right\}} \delta_{\gamma \backslash\left\{z^{\prime}\right\}, \gamma^{\prime}} \cdot \beta\right]
\end{aligned}
$$

при $\gamma \neq \varnothing$ и

$$
R_{\varnothing, z ; \gamma^{\prime}, z^{\prime}}=p_{0}\left(z-z^{\prime}\right) \delta_{\varnothing, \gamma^{\prime}}+c_{1}\left(z-z^{\prime}\right) \delta_{\left\{z^{\prime}\right\}, \gamma^{\prime}} .
$$

Здесь обозначено

$$
\mathbb{1}_{\{z \in \gamma\}}= \begin{cases}1, & z \in \gamma \\ 0, & z \notin \gamma\end{cases}
$$

и

$$
c_{1}(u)=\left\langle c(u ; \cdot) \cdot e_{1}(\cdot)\right\rangle_{\Pi},
$$

так что в силу $(0.5 \mathrm{a})$

$$
c(u ; s)=c_{1}(u) e_{1}(s)
$$

Далее

$$
\begin{gathered}
\alpha=\sum_{s} e_{1}^{3}(s) \pi(s), \quad \beta=\sum_{s, s^{\prime}} e_{1}(s) e_{1}\left(s^{\prime}\right) \Delta\left(s, s^{\prime}\right) \pi(s) \\
\varphi=\sum_{s, s^{\prime}} \pi(s) \Delta\left(s, s^{\prime}\right) e_{1}\left(s^{\prime}\right) .
\end{gathered}
$$

Разложение вектора $\Psi \in \mathscr{H}$ по базису $\left\{\Psi_{\gamma, z}\right\}$,

$$
\Psi=\sum_{\gamma, z} f_{\gamma, z} \Psi_{\gamma, z}
$$

порождает изоморфизм

$$
\Psi \rightleftharpoons\left\{f=\left(f_{\gamma, z}\right)\right\} \in l_{2}\left(\Gamma_{0} \times \mathbb{Z}^{\nu}\right)
$$


между пространствами $\mathscr{H}$ и $l_{2}\left(\Gamma_{0} \times \mathbb{Z}^{\nu}\right)$, где $\Gamma_{0}=\Gamma_{0}\left(\mathbb{Z}^{\nu}\right)$ - совокупность всех конечных подмножеств решетки $\mathbb{Z}^{\nu}$. При этом действие стохастического оператора, который мы по-прежнему обозначим $\mathscr{T}$, задается формулой

$$
(\mathscr{T} f)_{\gamma, z}=\sum_{\gamma^{\prime}, z^{\prime}} R_{\gamma^{\prime}, z^{\prime} ; \gamma, z} f_{\gamma^{\prime}, z^{\prime}}
$$

В пространстве $\mathscr{H}$ действует группа операторов трансляций

$$
\left(U_{v} \Psi\right)(\eta, x)=\Psi\left(\tau_{v}^{-1} \eta, x-v\right), \quad v \in \mathbb{Z}^{\nu},
$$

где $\tau_{v}$ - преобразования в пространстве $S^{\mathbb{Z}^{\nu}}$ :

$$
\left(\tau_{v} \eta\right)(u)=\eta(u+v), \quad v \in \mathbb{Z}^{\nu}, \quad \eta \in S^{\mathbb{Z}^{\nu}} .
$$

При переходе к пространству $l_{2}\left(\Gamma_{0} \times \mathbb{Z}^{\nu}\right)$ операторы $U_{v}$ принимают вид

$$
\left(U_{v} f\right)_{\gamma, z}=f_{\gamma-v, z-v}
$$

где $\gamma-v$ - сдвиг подмножества $\gamma$ на вектор $-v$. Очевидно, что оператор $\mathscr{T}$ и группа операторов $\left\{U_{v}, v \in \mathbb{Z}^{\nu}\right\}$ коммутируют друг с другом, и нам будет удобно перейти к представлению, в котором операторы $U_{v}$ "диагонализуются".

А именно, рассмотрим отображение

$$
W: l_{2}\left(\Gamma_{0} \times \mathbb{Z}^{\nu}\right) \rightarrow \int_{\mathbb{T}^{\nu}} \mathscr{H}(\lambda) d \lambda
$$

пространства $l_{2}\left(\Gamma_{0} \times \mathbb{Z}^{\nu}\right)$ в прямой интеграл пространств $\mathscr{H}(\lambda) \equiv l_{2}\left(\Gamma_{0}\right)[13]$; здесь $\mathbb{T}^{\nu}-\nu$-мерный тор с нормированной мерой Хаара $m(\lambda)$, а отображение $W$ действует по формуле

$$
\begin{gathered}
(W f)_{\lambda}(\gamma) \equiv \varphi_{\lambda}(\gamma)=\sum_{z \in \mathbb{Z}^{\nu}} f_{\gamma+z, z} e^{i(\lambda, z)}, \\
f=\left\{f_{\gamma, z}\right\} \in l_{2}\left(\Gamma_{0} \times \mathbb{Z}^{\nu}\right) .
\end{gathered}
$$

При этом отображении операторы $\mathscr{T}$ и $\left\{U_{v}, v \in \mathbb{Z}^{\nu}\right\}$ перейдут в прямые операторные интегралы:

$$
\mathscr{T} \rightarrow \int_{\mathbb{T}^{\nu}} \mathscr{T}(\lambda) d \lambda, \quad U_{v} \rightarrow \int_{\mathbb{T}^{\nu}} e^{i(\lambda, v)} E_{\lambda} d \lambda,
$$

где $E_{\lambda}=E$ - единичный оператор в $l_{2}\left(\Gamma_{0}\right)$, а $\left\{\mathscr{T}(\lambda), \lambda \in \mathbb{T}^{\nu}\right\}-$ семейство операторов в $l_{2}\left(\Gamma_{0}\right)$, действующих по формуле

$$
(\mathscr{T}(\lambda) \varphi)(\gamma)=\sum_{\gamma^{\prime} \in \Gamma} \widehat{R}_{\gamma, \gamma^{\prime}}(\lambda) \varphi\left(\gamma^{\prime}\right), \quad \varphi=\left\{\varphi(\gamma), \gamma \in \Gamma_{0}\right\} \in l_{2}\left(\Gamma_{0}\right),
$$

где

$$
\widehat{R}_{\gamma, \gamma^{\prime}}(\lambda)=\sum_{u \in \mathbb{Z}^{\nu}} R_{\gamma^{\prime}, 0 ; \gamma+u, u} e^{i(\lambda, u)}
$$

Благодаря вещественности матричных элементов $R_{\gamma, z ; \gamma^{\prime}, z^{\prime}}$, величины $\widehat{R}_{\gamma, \gamma^{\prime}}(\lambda)$ являются четными функциями $\lambda: \widehat{R}_{\gamma, \gamma^{\prime}}(-\lambda)=\widehat{\widehat{R}}_{\gamma, \gamma^{\prime}}(\lambda)$. Отображение $W$ унитарно и обратимо:

$$
\left(W^{-1} \varphi_{\lambda}\right)(\gamma, z)=\int_{\mathbb{T}^{\nu}} \varphi_{\lambda}(\gamma-z) e^{-i(\lambda, z)} d \lambda
$$


Мы опишем "старшую" часть спектра оператора $\mathscr{T}(\lambda)$ для $\lambda \in \mathbb{T}^{\nu}$, заключенных в некоторой небольшой окрестности точки $\lambda=0$. Для лучшего понимания возникающей картины рассмотрим семейство операторов $\mathscr{T}^{0}(\lambda)$, порожденное стохастическим оператором $\mathscr{T}^{0}$ для “невозмущенной” цепи $\left\{X_{t}, \eta_{t}, t \in \mathbb{Z}^{1}\right\}$, для которой $\varepsilon_{1}=\varepsilon_{2}=0$.

Из формул $(2.2 .7)$ и $(2.2 .11 \mathrm{~b}),(2.2 .11 \mathrm{c})$ видно, что в этом случае функция $\chi_{0}(\gamma)=\delta_{\gamma, \varnothing} \in l_{2}\left(\Gamma_{0}\right)$ является собственной функцией оператора $\mathscr{T}^{0}(\lambda)$ с собственным значением

$$
\tilde{p}_{0}(\lambda)=\sum_{u \in \mathbb{Z}^{\nu}} p_{0}(u) e^{i(\lambda, u)}
$$

а подпространство

$$
h^{0}=\left\{\varphi \in l_{2}\left(\Gamma_{0}\right): \varphi(\varnothing)=0\right\}
$$

инвариантно относительно $\mathscr{T}^{0}(\lambda)$ при любом $\lambda \in \mathbb{T}^{\nu}$ и этот оператор действует в $h^{0}$ по формуле

$$
\left(\mathscr{T}^{0}(\lambda) \varphi\right)(\gamma)=\mu^{|\gamma|} \sum_{u \in \mathbb{Z}^{\nu}} \varphi(\gamma-u) p_{0}(u) e^{i(\lambda, u)}, \quad \varphi \in h^{0}, \quad \gamma \neq \varnothing .
$$

Из этой формулы видно, что

$$
\left\|\left.\mathscr{T}^{0}(\lambda)\right|_{h^{0}}\right\|_{l_{2}\left(\Gamma_{0}\right)} \leqslant|\mu|
$$

и, следовательно, весь спектр $\left.\mathscr{T}^{0}(\lambda)\right|_{h^{0}}$ заключен в круге $|w|<|\mu|$ на комплексной плоскости $w$. Таким образом, для $\lambda$ из окрестности нуля

$$
O_{\delta}=\left\{\lambda \in \mathbb{T}^{\nu}:\left|\tilde{p}_{0}(\lambda)\right|>1-\delta\right\}, \quad \delta \leqslant \frac{1-|\mu|}{2},
$$

у оператора $\mathscr{T}^{0}(\lambda)$ имеется собственный вектор $\chi_{0} \in l_{2}\left(\Gamma_{0}\right)$ с собственным значением $\tilde{p}_{0}(\lambda)$, расположенным правее остального спектра этого оператора ${ }^{4}$. Как будет видно, аналогичная картина сохраняется и для полного ("возмущенного") оператора $\mathscr{T}(\lambda)$.

Введем пространство $l_{1}^{M}\left(\Gamma_{0}\right)$ функций $\varphi=\left\{\varphi(\gamma), \gamma \in \Gamma_{0}\right\}$ с нормой

$$
\|\varphi\|_{M}=\sum_{\gamma \in \Gamma_{0}}|\varphi(\gamma)| M^{|\gamma|}<\infty
$$

где $M=\max \left\{\max _{s \in S}\left|e_{1}(s)\right|, 2\right\}$. Очевидно, что пространство $l_{1}^{M}\left(\Gamma_{0}\right)$ содержится в пространстве $l_{2}\left(\Gamma_{0}\right)$ и всюду плотно в нем. Из выражений $(2.2 .7)$ для матричных элементов оператора $\mathscr{T}$ легко усмотреть, что при любом $\lambda$ пространство $l_{1}^{M}\left(\Gamma_{0}\right)$ инвариантно относительно оператора $\mathscr{T}(\lambda)$ и он ограничен относительно нормы $\|\cdot\|_{M}$.

В следующей лемме собраны все нужные факты, касающиеся спектральных свойств оператора $\mathscr{T}(\lambda)$. Доказательство леммы отнесено в приложение.

Лемма 2.2.1. Пусть $\varepsilon_{1}$ и $\varepsilon_{2}$ достаточно маль. Тогда:

1) $п р и \lambda \in O_{\delta}$

\footnotetext{
$1-\delta$.

${ }^{4}$ Мы полагаем $\delta>0$ столь малым, что вне окрестности $O_{\delta}$ выполнено неравенство $\tilde{p}_{0}(\lambda)<$
} 
i) оператор $\mathscr{T}(\lambda)$ имеет единственный собственный вектор $\chi_{\lambda} \in l_{1}^{M}\left(\Gamma_{0}\right)$ с собственным значением $\tilde{p}(\lambda)$ таким, что

$$
|\tilde{p}(\lambda)|>1-\frac{3}{2} \delta
$$

при этом $\chi_{\lambda}(\varnothing) \neq 0$ и при нормировке $\chi_{\lambda}(\varnothing)=1$ выполнено условие

$$
\sum_{\gamma \in \Gamma_{0}, \gamma \neq \varnothing}\left|\chi_{\lambda}(\gamma)\right| M^{|\gamma|}<\kappa
$$

әде $\kappa=\kappa\left(\varepsilon_{1}, \varepsilon_{2}\right)$ - достаточно малая константа, не зависящая от $\lambda$; кроме того,

$$
\chi_{\lambda=0}(\gamma)=\delta_{\gamma, \varnothing}
$$

и вектор $\chi_{\lambda}$ является аналитической функцией $\lambda \in O_{\delta}$ со значениями в $l_{1}^{M}\left(\Gamma_{0}\right)$;

ii) собственное значение $\tilde{p}(\lambda)$ обладает следующими свойствами:

а) выполнены соотношения

$$
\tilde{p}(0)=1, \quad|\tilde{p}(\lambda)|<1, \quad \lambda \neq 0,
$$

b) функция $\tilde{p}(\lambda)$ аналитична в $O_{\delta}$,

c) ряд Тейлора для $\log \tilde{p}(\lambda)$ в окрестности $\lambda=0$ имеет вид

$$
\log \tilde{p}(\lambda)=i \sum_{k=1}^{\nu} b_{k} \lambda_{k}-\frac{1}{2} \sum_{j, k} \bar{c}_{j k} \lambda_{j} \lambda_{k}+\cdots, \quad \lambda=\left(\lambda_{1}, \ldots, \lambda_{\nu}\right)
$$

где $b=\left\{b_{k}\right\}$ - вещественный вектор, а симметричная матрица $\bar{C}=\left\{\bar{c}_{j k}\right\}$ вещественна и соответствующая ей квадратичная форма положительно определена;

iii) у оператора $\mathscr{T}(\lambda)$ имеется инвариантное подпространство $h_{\lambda} \subset l_{1}^{M}\left(\Gamma_{0}\right)$ и справедливо разложение $l_{1}^{M}\left(\Gamma_{0}\right)$ в прлмую сумму подпространств:

$$
l_{1}^{M}\left(\Gamma_{0}\right)=\left\{c \chi_{\lambda}\right\}+h_{\lambda},
$$

где $\left\{c \chi_{\lambda}\right\}$ - одномерное пространство, натянутое на вектор $\chi_{\lambda} ;$ для нормы оператора $\left.\mathscr{T}(\lambda)\right|_{h_{\lambda}}$ выполнена оченка

$$
\left\|\left.\mathscr{T}(\lambda)\right|_{h_{\lambda}}\right\|_{l_{1}^{M}\left(\Gamma_{0}\right)}<|\mu|+\delta / 2<1
$$

iv) вектор $\chi_{\lambda}^{*}$, ортогональньй пространству $h_{\lambda}$ (относительно скалярного произведения в $\left.l_{2}\left(\Gamma_{0}\right)\right)$ и нормированный условием $\chi_{\lambda}^{*}(\varnothing)=1$, аналитичен по параметру $\lambda \in O_{\delta}$ и для него выполнена оченка, аналогичная $(2.2 .19 \mathrm{a})$;

2) $п р и$ $\lambda \notin O_{\delta}$

$$
\|\mathscr{T}(\lambda)\|_{l_{1}^{M}\left(\Gamma_{0}\right)}<1-\delta / 2 .
$$


2.3. Завершение доказательства теоремы 2.1.1. Вектору $\Psi_{\varnothing, x}$, входящему в выражение (2.2.5) для вероятности $\operatorname{Pr}\left(X_{T}=x \mid X_{0}=x_{0}\right)$, при изоморфизме (2.2.8d) соответствует вектор $f^{\varnothing, x}=\left\{f_{\gamma, z}^{\varnothing, x}=\delta_{\gamma, \varnothing} \delta_{z, x}\right\} \in l_{2}\left(\Gamma_{0} \times \mathbb{Z}^{\nu}\right)$. При отображении $W$ этот вектор переходит в семейство векторов:

$$
\left(W f^{\varnothing, x}\right)_{\lambda}(\gamma) \equiv \varphi_{\lambda}^{\varnothing, x}(\gamma)=\delta_{\gamma, \varnothing} e^{i(\lambda, x)}
$$

Очевидно, что при любом $\lambda$ векторы $\varphi_{\lambda}^{\varnothing, x}$ принадлежат пространству $l_{1}^{M}\left(\Gamma_{0}\right)$ и, таким образом, при $\lambda \in O_{\delta}$ имеет место разложение

$$
\varphi_{\lambda}^{\varnothing, x}=C^{\varnothing, x}(\lambda) \chi_{\lambda}+\bar{\varphi}_{\lambda}^{\varnothing, x}
$$

где $\bar{\varphi}_{\lambda}^{\varnothing, x} \in h_{\lambda}$ и $\sup _{\lambda}\left\|\bar{\varphi}_{\lambda}^{\varnothing, x}\right\|_{l_{1}^{M}\left(\Gamma_{0}\right)}=c_{1}<\infty$, а коэффициент

$$
C^{\varnothing, x}(\lambda)=\frac{\left(\varphi_{\lambda}^{\varnothing, x}, \chi_{\lambda}^{*}\right) l_{2}\left(\Gamma_{0}\right)}{\left(\chi_{\lambda}, \chi_{\lambda}^{*}\right) l_{2}\left(\Gamma_{0}\right)}=\frac{e^{i(\lambda, x)}}{1+\sum_{\gamma \neq \varnothing} \chi_{\lambda}(\gamma) \bar{\chi}_{\lambda}^{*}(\gamma)}
$$

в силу утверждений леммы (2.2.1) является аналитической функцией $\lambda \in O_{\delta}$. Итак, подынтегральное выражение в (2.2.5) представимо в виде трех слагаемых:

$$
\begin{aligned}
& \left(\mathscr{T}^{T} \Psi_{\varnothing, x}\right)\left(\eta, x_{0}\right)=\sum_{\gamma}\left(\mathscr{T}^{T} f_{0}^{\varnothing, x}\right)_{\gamma, x_{0}} \prod_{y \in \gamma} e_{1}(\eta(y)) \\
& \quad+\sum_{\gamma}\left(\mathscr{T}^{T} f_{1}^{\varnothing, x}\right)_{\gamma, x_{0}} \prod_{y \in \gamma} e_{1}(\eta(y))+\sum_{\gamma}\left(\mathscr{T}^{T} f_{2}^{\varnothing, x}\right)_{\gamma, x_{0}} \prod_{y \in \gamma} e_{1}(\eta(y)) .
\end{aligned}
$$

Здесь

$$
\begin{aligned}
f_{0}^{\varnothing, x} & =W^{-1} \int_{O_{\delta}} C^{\varnothing, x}(\lambda) \chi_{\lambda} d \lambda, \\
f_{1}^{\varnothing, x} & =W^{-1} \int_{O_{\delta}} \bar{\varphi}_{\lambda}^{\varnothing, x} d \lambda, \\
f_{2}^{\varnothing, x} & =W^{-1} \int_{\mathbb{T}^{\nu} \backslash O_{\delta}} \varphi_{\lambda}^{\varnothing, x} d \lambda .
\end{aligned}
$$

Оценим вклад второго слагаемого в (2.3.4). Имеем согласно (2.2.12)

$$
\left(\mathscr{T}^{T} f_{1}^{\varnothing, x}\right)_{\gamma, x_{0}}=\int_{O_{\delta}}\left(\mathscr{T}^{T}(\lambda) \bar{\varphi}_{\lambda}^{\varnothing, x}\right)\left(\gamma-x_{0}\right) e^{-i\left(\lambda, x_{0}\right)} d \lambda
$$

Отсюда, как следует из утверждения (2.2.22) леммы 2.2.1, получаем

$$
\begin{gathered}
\left|\sum_{\gamma}\left(\mathscr{T}^{T} f_{1}^{\varnothing, x}\right)_{\gamma, x_{0}} \prod_{y \in \gamma} e_{1}(\eta(y))\right| \leqslant \sum_{\gamma}\left|\left(\mathscr{T}^{T} f_{1}^{\varnothing, x}\right)_{\gamma, x_{0}}\right| M^{|\gamma|} \\
\quad=\int_{O_{\delta}} \sum_{\gamma}\left|\left(\mathscr{T}^{T}(\lambda) \bar{\varphi}_{\lambda}^{\varnothing, x}\right)(\gamma)\right| M^{|\gamma|} d \lambda \\
\leqslant C \max _{\lambda \in O_{\delta}}\left(\left\|\left.\mathscr{T}(\lambda)\right|_{h_{\lambda}}\right\|_{l_{1}^{M}\left(\Gamma_{0}\right)}\right)^{T}<C(|\mu|+\delta / 2)^{T}
\end{gathered}
$$

где $C=\int_{O_{\delta}} d \lambda$ - константа, не зависящая от $T$. 
Таким образом, вклад второго слагаемого в сумму (2.3.4) экспоненциально убывает при $T \rightarrow \infty$. Аналогично оценивается вклад третьего слагаемого в (2.3.4), который, благодаря оценке (2.2.23), оказывается экспоненциально малым. Наконец,

$$
\begin{aligned}
\sum_{\gamma}\left(\mathscr{T}^{T} f_{0}^{\varnothing, x}\right)_{\gamma, x_{0}} \prod_{y \in \gamma} e_{1}(\eta(y)) \\
\quad=\sum_{\gamma} \prod_{y \in \gamma} e_{1}(\eta(y)) \int_{O_{\delta}} \tilde{p}^{T}(\lambda) \frac{\chi_{\lambda}\left(\gamma-x_{0}\right) e^{i\left(\lambda,\left(x-x_{0}\right)\right)}}{1+\sum_{\bar{\gamma} \neq \varnothing} \chi_{\lambda}(\bar{\gamma}) \bar{\chi}_{\lambda}^{*}(\bar{\gamma})} d \lambda .
\end{aligned}
$$

Заметим, что в силу оценки (2.2.19a) ряд

$$
\sum_{\gamma} \frac{\chi_{\lambda}\left(\gamma-x_{0}\right)}{1+\sum_{\bar{\gamma} \neq \varnothing} \chi_{\lambda}(\bar{\gamma}) \bar{\chi}_{\lambda}^{*}(\bar{\gamma})} \prod_{y \in \gamma} e_{1}(\eta(y)) \equiv 1+g(\lambda ; \eta)
$$

абсолютно сходится и $g(\lambda ; \eta)$ - функция, аналитическая относительно $\lambda$ и равномерно ограниченная при всех $\eta \in S^{\mathbb{Z}^{\nu}}$, причем $g(0 ; \eta)=0$, как следует из (2.2.19b). Таким образом, (2.3.7) можно записать в виде

$$
\int_{O_{\delta}} \tilde{p}^{T}(\lambda)\left(1+g(\lambda ; \eta) e^{i\left(\lambda, x-x_{0}\right)} d \lambda .\right.
$$

Учитывая (2.2.20a), т. е. что $\max _{\lambda}|\tilde{p}(\lambda)|=\tilde{p}(0)=1$, мы стандартным образом выводим (см., например, [14]), что асимптотика интеграла $(2.3 .9)$ при $T \rightarrow \infty$ и при условии, что $\left|x-x_{0}-b T\right|=o\left(T^{2 / 3}\right)$, равна

$$
\frac{(\operatorname{det} A)^{1 / 2}}{(2 \pi T)^{\nu / 2}} \exp \left\{-\frac{1}{2} \mathscr{A}\left(\frac{x-x_{0}-b T}{\sqrt{T}}\right)\right\}\left(1+O\left(\frac{1}{\sqrt{T}}\right)\right),
$$

где $\mathscr{A}(v), v \in \mathbb{R}^{\nu},-$ квадратичная форма с матрицей $A=C^{-1}$, а матрица $C$ и вектор $b$ определены в (2.2.20b). Теорема 2.1.1 доказана.

\section{4. Другие темы (иной метод доказательства; поле с точки зрения частицы; убывание корреляций; большие уклонения).}

2.4.1. Изложенная выше annealed-модель изучалась в ряде работ. Самой ранней из них была статья [15], в которой, в частности, также была доказана центральная предельная теорема для положения блуждающей частицы. Метод, примененный в этой работе, - непосредственное кластерное разложение для вероятности траектории частицы (см. (0.7c)). Кроме центральной предельной теоремы в этой работе изучалась цепь

$$
\Delta_{t}=X_{t+1}-X_{t}, \quad t \leqslant 0,
$$

скачков частицы за один такт времени и было показано, что она удовлетворяет условию сильного перемешивания. Кроме того, показано, что последовательности средних и дисперсий

$$
m_{t}=\left\langle\Delta_{t}\right\rangle, \quad \sigma_{t}=\left\langle\left(\Delta_{t}-m_{t}\right)^{2}\right\rangle
$$

имеют экспоненциально убывающие при $t \rightarrow \infty$ приращения за один такт времени.

Далее, другой подход к центральной предельной теореме, изложенный выше и основанный на изучении старших ветвей спектра трансфер-матрицы (стохастического оператора) модели, был введен впервые в работе [16]. 
2.4.2. В последующих работах [17], [18] было изучено так называемое поле "с точки зрения частицы", а также убывание со временем $t$ корреляций различных локальных характеристик системы.

Поле "с точки зрения частицы” определяется следующим образом:

$$
\zeta_{t}(x)=\eta_{t}\left(x+X_{t}\right)
$$

где $X_{t}$ - положение блуждающей частицы в момент $t$. Это поле изучалось в $[17 ;$ ч. II].

В случаe quenched-модели случайного блуждания (т. е. при фиксированной реализации марковского поля $\eta)$ поле $\zeta_{t}(x)$ также является марковским. Нетрудно показать (см. снова [17; ч. II]), что в annealed-модели, рассмотренной в этом параграфе, поле $\zeta_{t}(x)$ также образует марковскую цепь. Пусть $\Pi_{t}-$ распределение значений поля $\zeta_{t}$ в момент $t$, порожденное начальным распределением П при $t=0$. Верна следующая теорема (см. [17; ч. II]).

ТЕОРема 2.4.1. Существует единственное распределение вероятностей Я на $\Omega=S^{\mathbb{Z}^{\nu}}$, инвариантное относительно марковской иепи $\zeta_{t}$, такое, что при достаточно малых $\varepsilon_{1}$ и $\varepsilon_{2}$ (см. (2.1.3)) для любого начального распределения П и любой ограниченной функции $F$ на $\Omega$ выполняется следующее неравенство:

$$
\left|\left\langle F\left(\zeta_{t}\right)\right\rangle_{\Pi_{t}}-\left\langle F\left(\zeta_{t}\right)\right\rangle_{\mathscr{P}}\right|<C_{F} e^{-\kappa t},
$$

где константа $C_{F}$, зависящая от функиии $F$, не зависит от начального распределения П, а константа $\kappa>0$ не зависит от $F u$ П. Кроме того, распределение Я абсолютно непрерывно относительно произведения мер $\Pi_{0}=\pi^{\nu}$ на $\Omega$ (мера $\pi$ - стационарная мера цепи (2.1.2), определенная на с. 45) и для любой иилиндрической функции $f$ на $\Omega$, зависящей от переменных поля $\{\zeta(x)$, $x \in \gamma\}, \gamma \subset \Omega,|\gamma|<\infty$, выполняется неравенство

$$
\left|\langle f\rangle_{\mathscr{P}}-\langle f\rangle_{\Pi_{0}}\right|<C_{f} q^{d(\gamma, 0)}
$$

где $C_{f}-$ константа, зависящая от $f, 0<q<1$ - константа, не зависящая oт $f u \gamma, d(\gamma, 0)$ - расстояние от начала координат до множества $\gamma$.

Для ограниченных функций $f_{1}, f_{2}$, зависящих лишь от значений поля $\zeta$ в двух фиксированных точках $x_{1}, x_{2} \in \mathbb{Z}^{\nu}$, в [17; ч. II] для случая $\nu \geqslant 3$ найдена точная асимптотика корреляций вида

$$
\left\langle f_{1}\left(\zeta_{t}\left(x_{1}\right)\right), f_{2}\left(\zeta_{0}\left(x_{2}\right)\right)\right\rangle_{\mathscr{P}_{\Pi}}=C \mu^{t} \frac{e^{-\alpha t}}{t^{\nu / 2}}(1+o(1)),
$$

где $\mathscr{P}_{\Pi}$ - распределение поля $\left\{\zeta_{t}(x)\right\}$ на $S^{\mathbb{Z}^{\nu+1}}$, порожденное начальным распределением П; $C>0$ - константа, зависящая от П, $f_{1}, f_{2}, x_{1}, x_{2} ; \mu$ - собственное значение оператора $(2.2 .1) ; \alpha>0$ зависит только от параметров модели. Последний результат был установлен и для случая $\nu=1$ в недавней работе [19] (использованный в этой работе метод может быть применен и для случая $\nu=2)$. Более точно, мы доказали, что в случае $\nu=1$ левая часть (2.4.3а) равна

либо $\frac{C_{1}}{t^{1 / 2}} e^{-\alpha t}(1+o(1)), \quad$ либо $C_{2} e^{-\alpha_{0} t}(1+o(1))$, 
где $0<\alpha_{0}<\alpha_{1}$ зависят лишь от параметров модели, а $C_{1}, C_{2}$ также и от функций $f_{1}$ и $f_{2}$ и начального распределения П. Этот результат легко переносится также и на случай, когда функции $f_{1}, f_{2}$ в $(2.4 .3 \mathrm{a})$ зависят от приращений $\Delta_{t}=X_{t+1}-X_{t}$ в положении частицы. В обеих работах [17; ч. II] и [19] результаты (2.4.3a) и (2.4.3b) были получены с помощью исследования старших ветвей спектра трансфер-матрицы поля $\left\{\zeta_{t}(x)\right\}$.

2.4.3. Далее, в работе [17; ч. I] изучено убывание корреляций для исходного поля $\left\{\eta_{t}\right\}$ :

$$
\left\langle f_{1}\left(\eta_{t}\left(x_{1}\right)\right), f_{2}\left(\eta_{0}\left(x_{2}\right)\right)\right\rangle
$$

где $f_{1}, f_{2}$ - две функции на множестве $S$, а $x_{1}, x_{2}$ - две фиксированные точки решетки $\mathbb{Z}^{\nu}$. В случае, когда вектор $b \neq 0$ (“снос"), корреляции вида (2.4.4) убывают экспоненциально со временем, а в случае $b=0$ для них найдена несколько неожиданная асимптотика

$$
\sim \frac{C}{t^{1+\nu / 2}}
$$

вместо асимптотики $\sim 1 / t^{\nu / 2}$, которая напрашивается из априорных соображений. Коэффициент $C$ в (2.4.5) зависит от точек $x_{1}, x_{2}$, функций $f_{1}, f_{2}$ в $(2.4 .4)$ и начального распределения поля $\left\{\eta_{t}\right\}$.

2.4.4. В статье [20] для annealed-модели, изученной в предыдущих разделах, рассматривалось распределение для больших уклонений $\sim T$ в положении частицы от своего среднего смещения за время $T$. Иными словами, изучалось распределение $\mu_{t}$ для величины

$$
y_{T}=X_{T} / T \in \mathbb{R}^{\nu}
$$

и были получены оценки, типичные в такой задаче:

$$
\mu_{T}(E)<e^{-T \inf _{v \in E} L(v)},
$$

где $E \subset \mathbb{R}^{\nu}$ - замкнутое множество, и

$$
\mu_{T}(G)>e^{-T \inf _{v \in G} L(v)},
$$

где $G \subset \mathbb{R}^{\nu}$ - открытое множество. Здесь $L(v)$ - так называемая функция действия - строго выпукла и аналитична. В доказательстве этого результата применяется кластерное разложение для вероятности траектории частицы $(0.7 \mathrm{c})$ и с его помощью выводится уравнение для производящей функции экспоненциальных моментов для величины $X_{T}-X_{0}$.

2.4.5. Наконец, в работах [16] и [18] для случайного блуждания двух частиц в случайной среде изучаются все те же вопросы (центральная предельная теорема, убывание корреляций), что и для случая блуждания одной частицы. Метод этих работ аналогичен методу предыдущих разделов: подробное исследование верхней части спектра трансфер-матрицы модели. 


\section{§ 3. Блуждание в марковском поле; quenched-модель}

\section{1. Усредненная модель и формулировка основного результата.}

Здесь мы будем придерживаться тех же предположений, что и в предыдущих параграфах: блуждание частицы управляется по-прежнему переходными вероятностями (0.2). Дополнительно мы предположим, что характеристическая функция $\tilde{p}_{0}(\lambda)$ распределения $p_{0}$ ни при каких $\lambda$ не обращается в нуль:

$$
\tilde{p}_{0}(\lambda) \neq 0 \text {. }
$$

Поле $\eta$ представляет собой набор независимых и одинаково распределенных марковских цепей $\left\{\eta_{t}(x), x \in \mathbb{Z}^{\nu}\right\}$, приписанных каждой точке решетки $\mathbb{Z}^{\nu}$ и принимающих два значения \pm 1 . Матрицей переходных вероятностей каждой цепи служит матрица $\widehat{Q}=\left\{\hat{q}\left(s^{\prime}, s\right)\right\}$ (см. (2.1.2)). Как и в предыдущем параграфе, мы будем считать, что параметр $\varepsilon_{1}$ (см. (2.1.3)) достаточно мал; кроме того, дополнительно предположим, что собственное значение $\mu \neq 1$ матрицы $\widehat{Q}$ также мало: $\mu \ll 1$, и, в частности, выполнено условие

$$
\max _{\lambda \in \mathbb{T}^{\nu}} \frac{|\mu|}{\left|\tilde{p}_{0}(\lambda)\right|}<1 .
$$

Подобно предыдущим параграфам, здесь для описанной модели будет установлена центральная предельная теорема для положения $X_{T}$ частицы при больших $T$.

Прежде всего заметим, что для “усредненного” блуждания частицы в изучаемой модели вероятности $\operatorname{Pr}^{\text {уср. }}\left(\chi_{T}\right)=\left\langle\operatorname{Pr}\left(\chi_{T} ; \eta\right)\right\rangle_{\eta}\left(\chi_{T}-\right.$ траектория частицы $)$ в точности такие же, как и в частном случае annealed-модели из предыдущего параграфа, когда эволюция поля не зависит от блуждающей частицы, т. е. матрица $Q_{0}=\widehat{Q}$ (см. (2.1.1)). Таким образом, для "усредненного" блуждания в марковском поле центральная предельная теорема формулируется в точности так же, как теорема 2.1.1. Оказывается, верна теорема, аналогичная теореме 1.1.1 для quenched-модели в случае независимого поля.

ТЕОРема 3.1.1. Для случайного блуждания частицы в описанном выше марковском поле в случае размерности $\nu \geqslant 3$ при выполнении условий i)-iv) относительно переходных вероятностей для частицы (см. §1), а также условий (3.1.1) и при достаточно малом $\varepsilon_{1}\left(=\max _{u, s}|c(u ; s)|\right)$ для вероятности

$$
\operatorname{Pr}\left(X_{T} \in \sqrt{T} G+b T \mid X_{0}=0 ; \eta\right),
$$

где $G$ - ограниченная область с кусочно гладкой границей, верна асимптотика (0.9) (см. введение). Сходимость здесь понимается как сходимость для почти всех конфигураций поля $\eta \in \Omega=S^{\mathbb{Z}^{\nu+1}}$, а также как сходимость в метрике пространства $L_{2}(\Omega, \rho)$ ( $\rho$ - распределение для поля). При этом вектор $b$ в (3.1.1c) и матрица $A=\left\{a_{i j}\right\}$, задающие гауссову плотность $g(u)$, такие же, как и для аппеаlеd-модели из предыдущего параграфа (при условии $\left.Q_{0}=\widehat{Q}, c \mathcal{M} .(2.1 .1)\right)$.

Как и в случае quenched-модели в независимом поле, мы докажем здесь только часть этой теоремы, относящуюся к $L_{2}$-сходимости. Общий случай рассмотрен в работе [21]. 


\section{2. Начало доказательства теоремы 3.1.1; основные конструкции.}

3.2.1. Мультипликативный базис. Обозначим через $\widehat{\Omega}_{x}=S^{\mathbb{Z}^{1}}$ множество конфигураций цепи $\eta_{x}=\left\{\eta_{x}(t), t \in \mathbb{Z}^{1}\right\}$, приписанной точке $x \in \mathbb{Z}^{\nu}$. Очевидно, что все пространство конфигураций имеет вид

$$
\Omega=\prod_{x \in \mathbb{Z}^{\nu}} \widehat{\Omega}_{x}
$$

а распределение значений всего поля $\eta=\left\{\eta(t, x),(t, x) \in \mathbb{Z}^{1} \times \mathbb{Z}^{\nu}\right\}$ представимо в виде

$$
\rho=\prod_{x \in \mathbb{Z}^{\nu}} \hat{\rho}_{x}
$$

где $\hat{\rho}_{x}$ - распределение в пространстве $\widehat{\Omega}_{x}$ отдельной цепи $\eta_{x}$. Согласно этому

$$
L_{2}(\Omega, \rho)=\bigotimes_{x \in \mathbb{Z}^{\nu}} L_{2}\left(\widehat{\Omega}_{x}, \hat{\rho}_{x}\right)
$$

- тензорное произведение пространств с выделенными элементами $\mathbf{1}_{x}\left(\eta_{x}\right) \equiv 1$. Мы построим специальный мультипликативный базис в пространстве $L_{2}(\Omega, \rho)$, построив его предварительно в каждом множителе в (3.2.1). Для фиксированного $x \in \mathbb{Z}^{\nu}$ введем функции на пространстве $\widehat{\Omega}_{x}$ :

$$
\tau_{x, t}(\eta)=e_{1}\left(\eta_{x}(t)\right)-\left\langle e_{1}\left(\eta_{x}(t)\right) \mid \bar{\eta}_{x}(s), s<t\right\rangle_{\hat{\rho}_{x}},
$$

где $e_{1}$ - собственный вектор оператора $\widehat{\mathscr{T}}$ (см. (2.2.1)) с собственным значением $\mu, \mathrm{a}\left\langle\cdot \mid \bar{\eta}_{x}(s), s<t\right\rangle_{\hat{\rho}_{x}}-$ условное среднее, определяемое распределением $\hat{\rho}_{x}$ при условии, что значения цепи $\eta_{x}$ в моменты $s<t$ фиксированы. Легко видеть, что

$$
\tau_{x, t}\left(\eta_{x}\right)=e_{1}\left(\eta_{x}(t)\right)-\mu e_{1}\left(\eta_{x}(t-1)\right) .
$$

Нетрудно подсчитать, что условное среднее этой величины равно

$$
\left\langle\left(\tau_{x, t}\left(\eta_{x}\right)\right)^{2} \mid \bar{\eta}_{x}(s), s<t\right\rangle_{\rho}=1-\mu^{2}+\alpha \mu(1-\mu) e_{1}\left(\bar{\eta}_{x}(t-1)\right):=n^{2}\left(\eta_{x}(t-1)\right),
$$

где $\alpha=\sum_{s} e_{1}^{3}(s) \pi(s)$ - коэффициент в разложении $e_{1}^{2}(s)=\alpha e_{1}(s)+1$. Далее введем нормированную функцию

$$
\hat{v}_{x, t}\left(\eta_{x}\right)=\frac{\tau_{x, t}\left(\eta_{x}\right)}{n\left(\eta_{x}(t-1)\right)}
$$

и для любого $\widehat{B} \subset \mathbb{Z}^{1},|\widehat{B}|<\infty$, определим функцию

$$
\widehat{V}_{\widehat{B}}^{(x)}\left(\eta_{x}\right)=\prod_{t \in \widehat{B}} \hat{v}_{x, t}\left(\eta_{x}\right) .
$$

Можно проверить (см. [22]), что набор функций $\left\{\widehat{V}_{\widehat{B}}, \widehat{B} \in \Gamma_{0}\left(\mathbb{Z}^{1}\right)\right\}$ является ортонормированным базисом в пространстве $L_{2}\left(\widehat{\Omega}_{x}, \hat{\rho}_{x}\right)$ (здесь $\widehat{\Omega}_{x}=\Gamma_{0}\left(\mathbb{Z}^{1}\right)-$ совокупность конечных подмножеств решетки $\left.\mathbb{Z}^{1}\right)$. Наконец, для любого конечного множества $B \subset \mathbb{Z}^{\nu+1}$ определим функцию

$$
V_{B}(\eta)=\prod_{x \in \mathbb{Z}^{\nu}} \widehat{V}_{\widehat{B}(x)}^{(x)}\left(\eta_{x}\right), \quad V_{\varnothing}(\eta)=1,
$$

где $\widehat{B}(x)=\left\{t \in \mathbb{Z}^{1}:(x, t) \in B\right\}$. Функции $\left\{V_{B}, B \in \Gamma_{0}\left(\mathbb{Z}^{\nu+1}\right)\right\}$ образуют ортонормированный базис в $L_{2}(\Omega, \rho)$. 
3.2.2. Разложение вероятности $\operatorname{Pr}\left(\chi_{T} ; \eta\right)$ по базису $\left\{V_{B}\right\}$. Траекторию случайного блуждания частицы

$$
\chi_{T}=\left(X_{0}, X_{1}, \ldots, X_{T}\right)
$$

за $T$ шагов удобно представлять себе как последовательность

$$
\chi_{T}=\left(b_{0}, b_{1}, \ldots, b_{T-1}\right)
$$

ребер $b_{t}=\left(t, X_{t}, \Delta_{t}\right)$, соединяющих две последовательные вершины траектории $X_{t}$ и $X_{t+1}, \Delta_{t}=X_{t+1}-X_{t}$. Вероятность траектории $\chi_{T}$, как мы уже видели (см. $(0.7 \mathrm{c}))$, при фиксированной конфигурации поля запишется (с учетом (2.2.8b)) в виде

$$
\operatorname{Pr}\left(\chi_{T} ; \eta\right)=\prod_{t=0}^{T-1}\left(p_{0}\left(\Delta_{t}\right)+c_{1}\left(\Delta_{t}\right) e_{1}\left(\eta\left(X_{t}, t\right)\right)\right) .
$$

Разложим эту вероятность (как функцию поля $\eta$ ) по базису $\left\{V_{B}\right\}$ :

$$
\operatorname{Pr}\left(\chi_{T} ; \eta\right)=\left\langle\operatorname{Pr}\left(\chi_{T} ; \eta\right)\right\rangle_{\eta}+\sum_{\substack{B \in \Gamma_{0}\left(\mathbb{Z}^{\nu+1}\right) \\ B \neq \varnothing}} N\left(\chi_{T}, B\right) V_{B}
$$

где

$$
N\left(\chi_{T}, B\right)=\left(\operatorname{Pr}\left(\chi_{T} ; \eta\right), V_{B}(\eta)\right)
$$

a $(\cdot, \cdot)$ - скалярное произведение в $L_{2}(\Omega, \rho)$. Так как оба множителя в (3.2.4а) представляют собой произведение функций от поля, мы можем воспользоваться хорошо известной формулой, выражающей моменты случайных величин через их семиинварианты (см., например, [23]). Поскольку семиинварианты вида $\left\langle\prod_{(x, t) \in B}^{\prime} \hat{v}_{x, t}(\eta)\right\rangle$ (в обозначениях из [23]) обращаются в нуль:

$$
\left\langle\prod_{(x, t) \in B}^{\prime} \hat{v}_{x, t}(\eta)\right\rangle=0
$$

что следует из равенства нулю всех моментов $\left\langle\prod_{(x, t) \in B} \hat{v}_{x, t}(\eta)\right\rangle, B \neq \varnothing$, то в разложение $N\left(\chi_{T}, B\right)$ войдут семиинварианты, имеющие вид

$$
\begin{array}{r}
\left\langle\prod_{(x, t) \in \bar{B}}^{\prime} \hat{v}_{x, t}(\eta), \prod_{b_{t} \in L}^{\prime}\left(p_{0}\left(\Delta_{t}\right)+c_{1}\left(\Delta_{t}\right) e_{1}\left(\eta\left(X_{t}, t\right)\right)\right)\right\rangle \\
\quad=\prod_{b_{t} \in L} c_{1}\left(\Delta_{t}\right)\left\langle\prod_{(x, t) \in \bar{B}}^{\prime} v_{x, t}(\eta), \prod_{b_{t} \in L}^{\prime} e_{1}\left(\eta\left(X_{t}, t\right)\right)\right\rangle .
\end{array}
$$

Здесь $\bar{B} \subseteq B$, а $L=\left\{b_{t} \in \chi_{T}\right\}$ - некоторое подмножество ребер траектории $\chi_{T}$. Для каждого такого подмножества $L$ обозначим через $\varphi_{L}$ совокупность всех 
его (неупорядоченных) разбиений $q_{L}=\left\{L_{1}, L_{2}, \ldots, L_{s}\right\}$ на попарно непересекающиеся части и запишем

$$
\begin{aligned}
N\left(\chi_{T}, B\right)= & \sum_{L \subseteq \chi_{T}} \sum_{q_{L} \in \varphi_{L}} \prod_{i=1}^{s}\left\langle\prod_{b_{t} \in L_{i}}^{\prime}\left(p_{0}\left(\Delta_{t}\right)+c_{1}\left(\Delta_{t}\right) e_{1}\left(\eta\left(X_{t}, t\right)\right)\right)\right\rangle \\
& \times \prod_{b_{t} \in L^{\prime}} c_{1}\left(\Delta_{t}\right) \sum_{q_{B, L^{\prime}} \in \varphi_{B, L^{\prime}}} \prod_{j=1}^{m}\left\langle\prod_{(x, t) \in B_{j}}^{\prime} \hat{v}_{(x, t)}(\eta), \prod_{b_{t} \in L_{j}^{\prime}}^{\prime} e_{1}\left(\eta\left(X_{t}, t\right)\right)\right\rangle .
\end{aligned}
$$

Здесь $L^{\prime}=\chi_{T} \backslash L$, а $\varphi_{B, L^{\prime}}-$ совокупность всех разбиений $q_{B, L^{\prime}}=\left\{\left(B_{1}, L_{1}^{\prime}\right)\right.$, $\left.\left(B_{2}, L_{2}^{\prime}\right), \ldots,\left(B_{m}, L_{m}^{\prime}\right)\right\}, m=\left|q_{B, L^{\prime}}\right|$, пары множеств $\left(B, L^{\prime}\right)$ в пары их подмножеств. Подмножество $L \subseteq \chi_{T}$ назовем свободной, а подмножество $L^{\prime} \subseteq \chi_{T}-$ взаимодействующей частями траектории $\chi_{T}$. Для любого $L \subset \chi_{T}$

$$
\begin{aligned}
\sum_{q_{L} \subset \varphi_{L}} & \prod_{i=1}^{s}\left\langle\prod_{b_{t} \in L_{i}}^{\prime}\left(p_{0}\left(\Delta_{t}\right)+c_{1}\left(\Delta_{t}\right) e_{1}\left(\eta\left(x_{t}, t\right)\right)\right)\right\rangle \\
\quad & =\left\langle\prod_{b_{t} \in L}\left(p_{0}\left(\Delta_{t}\right)+c_{1}\left(\Delta_{t}\right) e_{1}\left(\eta\left(x_{t}, t\right)\right)\right)\right\rangle=\langle P(L ; \eta)\rangle:=\widehat{P}(L) .
\end{aligned}
$$

Для любой пары $\left(B, B^{\prime}\right)$ подмножеств решетки $\mathbb{Z}^{\nu+1}$ через $\omega_{B, B^{\prime}}$ обозначим семиинвариант

$$
\left\langle\prod_{(x, t) \in B}^{\prime} v_{x, t}(\eta), \prod_{\left(x^{\prime}, t^{\prime}\right) \in B^{\prime}}^{\prime} e_{1}\left(\eta\left(x^{\prime}, t^{\prime}\right)\right)\right\rangle .
$$

Этот семиинвариант может быть отличен от нуля лишь в случае, когда "пространственные" проекции множеств $B$ и $B^{\prime}$ состоят каждая из одной точки, эти точки совпадают и, кроме того, самая "поздняя" точка из $B$ (т.е. точка $(x, t) \in B$ с наибольшим $t)$ предшествует по времени какой-нибудь точке $\left(x, t^{\prime}\right) \in B^{\prime}$ (т. е. $\left.t<t^{\prime}\right)$. Обозначая $B_{L^{\prime}}^{\prime}=\left\{\left(X_{t^{\prime}}, t^{\prime}\right), b_{t^{\prime}} \in L^{\prime}\right\}$ - множество начальных точек всех ребер из $L^{\prime}$, можно записать $N\left(\chi_{T}, B\right)$ в виде $(B \neq \varnothing)$

$$
N\left(\chi_{T}, B\right)=\sum_{L \subset \chi_{T}} \widehat{P}(L) \prod_{b_{t} \in L^{\prime}} c_{1}\left(\Delta_{t}\right) \sum_{q_{B, B_{L}^{\prime}}, \in \varphi_{B, B_{L^{\prime}}^{\prime}}} \prod_{j=1}^{m} \omega_{B_{j}, B_{j}^{\prime}},
$$

где $\varphi_{B, B_{L^{\prime}}^{\prime}}$ - совокупность всех разбиений $q_{B, B_{L}^{\prime}}=\left\{\left(B_{1}, B_{1}^{\prime}\right), \ldots,\left(B_{m}, B_{m}^{\prime}\right)\right\}$ пары множеств $\left(B, B_{L^{\prime}}^{\prime}\right)$. Таким образом, окончательно

$$
\begin{aligned}
\operatorname{Pr}\left(\chi_{T} ; \eta\right)=\langle & \left.\operatorname{Pr}\left(\chi_{T} ; \eta\right)\right\rangle_{\eta}+\sum_{L \subset \chi_{T}} \widehat{P}(L) \prod_{b_{t} \in L^{\prime}} c_{1}\left(\Delta_{t}\right) \\
& \times \sum_{B \neq \varnothing} \sum_{q_{B, B_{L^{\prime}}^{\prime}} \in \varphi_{B, B_{L^{\prime}}^{\prime}}} \prod_{j=1}^{m} \omega_{B_{j}, B_{j}^{\prime}} V_{B} .
\end{aligned}
$$

3.2.3. Окончательное представление для $\operatorname{Pr}\left(X_{T}=y \mid X_{0}=0 ; \eta\right)$. Разложим части траектории $L$ и $L^{\prime}$ на связанные серии подряд идущих ребер:

$$
L=\chi_{0} \cup \chi_{1} \cup \cdots \cup \chi_{k}, \quad L^{\prime}=\chi_{1}^{\prime} \cup \chi_{2}^{\prime} \cup \cdots \cup \chi_{k}^{\prime},
$$


занумерованных в хронологическом порядке. Вся траектория $\chi_{T}$ представится таким образом как чередующаяся последовательность серий:

$$
\chi_{T}=\chi_{0} \cup \chi_{1}^{\prime} \cup \chi_{1} \cup \chi_{2}^{\prime} \cup \chi_{2} \cup \cdots \cup \chi_{k}^{\prime} \cup \chi_{k}
$$

так, что начальная точка серии $\chi_{i}$ (или $\chi_{i}^{\prime}$ ), а именно $\left(t_{0}\left(\chi_{i}\right), y_{0}\left(\chi_{i}\right)\right)$ (или $\left.\left(t_{0}\left(\chi_{i}^{\prime}\right), y_{0}\left(\chi_{i}^{\prime}\right)\right)\right)$, совпадает с конечной точкой $\left(t_{f}\left(\chi_{i}^{\prime}\right), y_{f}\left(\chi_{i}^{\prime}\right)\right)$, т. е. с концом последнего ребра серии $\chi_{i}^{\prime}$ (или с конечной точкой $\left(t_{f}\left(\chi_{i-1}\right), y_{f}\left(\chi_{i-1}\right)\right)$ серии $\left.\chi_{i-1}\right)$. В случае, когда последовательность (3.2.6) фактически начинается со штрихованной серии (или кончается такой серией), мы для единообразия обозначений положим $\chi_{0}=\varnothing$ (или $\left.\chi_{k}=\varnothing\right)$; см. рис. 3 .

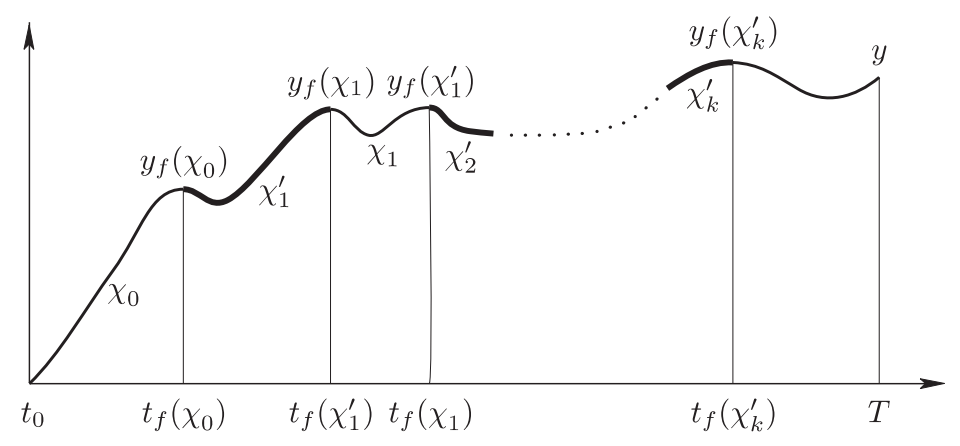

Рис. 3. Серии, на которые разбиваются части $L$ и $L^{\prime}$ траектории $\chi_{T}$

Зафиксируем значения поля $\eta$ в каждый начальный момент $t_{0}\left(\chi_{i}\right), i=$ $0,1, \ldots, k$, нештрихованной (“свободной”) серии:

$$
\eta_{t_{0}\left(\chi_{i}\right)}=\eta\left(t_{0}\left(\chi_{i}\right), y_{0}\left(\chi_{i}\right)\right)=: \bar{\eta}^{(i)}, \quad i=0,1, \ldots, k
$$

и запишем

$$
\widehat{P}(L)=\langle P(L ; \eta)\rangle=\int\left\langle P(L ; \eta) \mid \bar{\eta}^{(0)}, \bar{\eta}^{(1)}, \ldots, \bar{\eta}^{(k)}\right\rangle d \rho\left(\bar{\eta}^{(0)}, \ldots, \bar{\eta}^{(k)}\right),
$$

где $\left\langle\cdot \mid \bar{\eta}^{(0)}, \ldots, \bar{\eta}^{(k)}\right\rangle$ - условное среднее при фиксированных значениях (3.2.7), а $\rho\left(\bar{\eta}^{(0)}, \ldots, \bar{\eta}^{(k)}\right)$ - распределение вероятностей этих значений. Далее в силу марковского свойства поля $\eta$

$$
\left\langle P(L ; \eta) \mid \bar{\eta}^{(0)}, \ldots, \bar{\eta}^{(k)}\right\rangle=\prod_{i=0}^{k-1}\left\langle P\left(\chi_{i} ; \eta\right) \mid \bar{\eta}^{(i)}, \bar{\eta}^{(i+1)}\right\rangle\left\langle P\left(\chi_{k} ; \eta\right) \mid \bar{\eta}^{(k)}\right\rangle
$$

где согласно обозначению (3.2.4b)

$$
P\left(\chi_{i} ; \eta\right)=\prod_{b_{t} \in \chi_{i}}\left(p_{0}\left(\Delta_{t}\right)+c_{1}\left(\Delta_{t}\right) e_{1}\left(\eta\left(x_{t}, t\right)\right)\right),
$$

a $\left\langle\cdot \mid \bar{\eta}^{(i)}, \bar{\eta}^{(i+1)}\right\rangle$ и $\left\langle\cdot \mid \bar{\eta}^{(k)}\right\rangle$ - условные средние при фиксированных $\bar{\eta}^{(i)}, \bar{\eta}^{(i+1)}$, $i=0,1, \ldots, k-1$, и соответственно $\bar{\eta}^{(k)}$. 
Далее зафиксируем множество $L^{\prime}$ (и тем самым все штрихованные серии в представлении (3.2.6)) и для каждого $i=0,1, \ldots, k-1$ сумму по всевозможным путям (т. е. положениям серии $\chi_{i}$, см. рис. 3 ) обозначим следующим образом:

$$
\widehat{P}_{\bar{\eta}^{(i)}, \bar{\eta}^{(i+1)}}\left(s_{i}, x_{i} ; t_{i}, y_{i}\right)=\sum_{\substack{\chi_{i}:\left(t_{0}\left(\chi_{i}\right), y_{0}\left(\chi_{i}\right)\right)=\left(s_{i}, x_{i}\right) \\\left(t_{f}\left(\chi_{i}\right), y_{f}\left(\chi_{i}\right)\right)=\left(t_{i}, y_{i}\right)}}\left\langle P\left(\chi_{i} ; \eta\right) \mid \bar{\eta}^{(i)}, \bar{\eta}^{(i+1)}\right\rangle
$$

и

$$
\widehat{P}_{\bar{\eta}^{(k)}}\left(s_{k}, x_{k} ; T, y\right)=\sum_{\substack{\chi_{k}:\left(t_{0}\left(\chi_{k}\right), y_{0}\left(\chi_{k}\right)\right)=\left(s_{k}, x_{k}\right) \\\left(t_{f}\left(\chi_{k}\right), y_{f}\left(\chi_{k}\right)\right)=(T, y)}}\left\langle P\left(\chi_{k} ; \eta\right) \mid \bar{\eta}^{(k)}\right\rangle .
$$

При этом из соотношения $X_{0}=0$ следует, что $s_{0}=0, x_{0}=0$. В случае, когда $t_{0}=0$ (т. е. серия $\left.\chi_{0}=\varnothing\right)$, мы положим

$$
\widehat{P}_{\bar{\eta}^{(0)}, \bar{\eta}^{(1)}}\left(s_{0}=0, x_{0}=0 ; t_{0}=0, y_{0}\right)=\delta_{y_{0}, 0}
$$

и аналогичное соглашение при $\chi_{k}=\varnothing$ :

$$
\widehat{P}_{\bar{\eta}^{(k)}}\left(s_{k}=T, x_{k} ; T, y\right)=\delta_{x_{k}, y} .
$$

Определим еще величину

$$
h_{\bar{\eta}^{(k)}}\left(s_{k}-1, \bar{y}_{k} ; T, y\right)=\sum_{u \in \mathbb{Z}^{\nu}} c_{1}(u) P_{\bar{\eta}^{(k)}}\left(s_{k}, \bar{y}+u ; T, y\right),
$$

где $\left(s_{k}-1, \bar{y}_{k}\right)$ - левый конец последнего ребра в серии $\chi_{k}^{\prime}$.

Далее пусть

$$
\mathscr{M}_{B^{\prime}}(\eta)=\prod_{i=0}^{k-1} \widehat{P}_{\bar{\eta}^{(i)}, \bar{\eta}^{(i+1)}}\left(s_{i}, x_{i} ; t_{i}, y_{i}\right)
$$

и

$$
M_{B^{\prime}}(y, T)=\int \mathscr{M}_{B^{\prime}}(\eta) h_{\bar{\eta}^{(k)}}\left(s_{k}-1, \bar{y}_{k} ; T, y\right) d \rho\left(\bar{\eta}^{(0)}, \ldots, \bar{\eta}^{(k)}\right) \prod_{b_{t} \in L^{\prime} \backslash b_{s_{k}-1}} c_{1}\left(\Delta_{t}\right)
$$

В этих обозначениях

$$
\operatorname{Pr}\left(X_{T}=y \mid X_{0}=0 ; \eta\right)=\operatorname{Pr}^{\text {ycp. }}\left(X_{T}=y \mid X_{0}=0\right)+\sum_{B, B^{\prime}} A_{B, B^{\prime}} M_{B^{\prime}}(y, T) V_{B}(\eta),
$$

где

$$
A_{B, B^{\prime}}=\sum_{q_{B, B^{\prime}} \in \varphi_{B, B^{\prime}}} \prod_{\left(B_{j}, B_{j}^{\prime}\right) \in q_{B, B^{\prime}}} \omega_{B_{j}, B_{j}^{\prime}},
$$

если $B \neq \varnothing$ и $B^{\prime}=B_{L^{\prime}}^{\prime}$ для набора ребер $L^{\prime}$, являющегося частью какой-нибудь траектории $\chi_{T}$ (назовем такие $B^{\prime}$ допустимыми), и

$$
A_{B, B^{\prime}}=0
$$

в противном случае.

Поскольку, как мы знаем из предыдущего параграфа (см. следствие из теоремы 2.1.1), асимптотика $\operatorname{Pr}^{\text {уср. }}\left(X_{T}=y \mid X_{0}=0\right)$ при $T \rightarrow \infty$ приводит к центральной предельной теореме в интегральной форме, нам следует подробнее исследовать второе слагаемое в (3.2.8c). 
3.3. Завершение доказательства теоремы 3.1.1. Рассмотрим функционалы

$$
\begin{aligned}
& F_{T}(f ; \eta)=\sum_{y \in \mathbb{Z}^{\nu}} \operatorname{Pr}\left(X_{T}=y \mid X_{0}=0 ; \eta\right) f\left(\frac{y-b T}{\sqrt{T}}\right), \\
& F_{T}^{\text {ycp. }}(f)=\sum_{y \in \mathbb{Z}^{\nu}} \operatorname{Pr}^{\text {ycp. }}\left(X_{T}=y \mid X_{0}=0\right) f\left(\frac{y-b T}{\sqrt{T}}\right),
\end{aligned}
$$

где $f \in C_{b}\left(\mathbb{R}^{\nu}\right)$ - ограниченная и непрерывная функция, а $b \in \mathbb{R}^{\nu}$ - средний снос частицы для усредненной модели (см. (3.1.1c)). Для любого допустимого множества $B^{\prime}$ с $t_{f}\left(B^{\prime}\right)=t$ и $y_{f}\left(B^{\prime}\right)=\bar{y}$ положим

$$
\widehat{M}_{B^{\prime}}(T, f)=\sum_{y \in \mathbb{Z}^{\nu}} M_{B^{\prime}}(y, T) f\left(\frac{y-T b}{\sqrt{T}}\right)=\int d \rho(\eta) M_{B^{\prime}}(t, \bar{y}) G_{T, f}\left(t, \bar{y} ; \bar{\eta}^{(k)}\right),
$$

где

$$
G_{T, f}\left(t, \bar{y} ; \bar{\eta}^{(k)}\right)=\sum_{y} h_{\bar{\eta}^{(k)}}(t, \bar{y} ; T, y) f\left(\frac{y-b T}{\sqrt{T}}\right)
$$

и $M_{B^{\prime}}(t, \bar{y})$ определяется аналогично $(3.2 .8 \mathrm{~b})$, только без множителя $h_{\bar{\eta}^{(k)}}\left(s_{k}-1\right.$, $\bar{y} ; T, y)$ под знаком интеграла. Из (3.2.8c) следует, что

$$
\mathscr{M}_{T}(f ; \eta):=F_{T}(f ; \eta)-F_{T}^{\mathrm{ycp} \cdot}(f)=\sum_{B, B^{\prime}} A_{B, B^{\prime}} \widehat{M}_{B^{\prime}}(T, f) V_{B}(\eta) .
$$

В силу ортонормированности базиса $\left\{V_{B}\right\}$

$$
\left\langle\mathscr{M}_{T}^{2}(f ; \eta)\right\rangle=\sum_{B}\left(\sum_{B^{\prime}} A_{B, B^{\prime}} \widehat{M}_{B^{\prime}}(T, f)\right)^{2} .
$$

Введем в пространстве $l_{2}\left(\Gamma_{0}\left(\mathbb{Z}^{\nu+1}\right)\right)$ линейный оператор

$$
\left(\mathscr{A}^{(\lambda)} g\right)_{B}=\sum_{B^{\prime}} A_{B, B^{\prime}} \lambda^{-\left|B^{\prime}\right|} g_{B^{\prime}}
$$

где $g=\left\{g_{B^{\prime}}\right\} \in l_{2}\left(\Gamma_{0}\left(\mathbb{Z}^{\nu+1}\right)\right)$, а $\lambda$ - параметр.

Лемма 3.3.1. При малом $\lambda^{-1}$ норма оператора $\mathscr{A}^{(\lambda)}$ менъше единицъ:

$$
\left\|\mathscr{A}^{(\lambda)}\right\|_{l_{2}\left(\Gamma_{0}\left(\mathbb{Z}^{\nu+1}\right)\right)}<1 .
$$

Доказательство этой леммы приведено в приложении. Таким образом, если (3.3.2b) переписать в виде

$$
\left\langle\mathscr{M}_{T}^{2}(f ; \eta)\right\rangle=\sum_{B \in \Gamma_{0}\left(\mathbb{Z}^{\nu+1}\right)}\left(\mathscr{A}^{(\lambda)} M^{(\lambda)}(T, f)\right)_{B}^{2},
$$

где

$$
M^{(\lambda)}(T, f)=\left\{\lambda^{\left|B^{\prime}\right|} \widehat{M}_{B^{\prime}}(T, f)\right\} \in l_{2}\left(\Gamma_{0}\left(\mathbb{Z}^{\nu+1}\right)\right),
$$

то получим, что

$$
\left\langle\mathscr{M}_{T}^{2}(f ; \eta)\right\rangle \leqslant\left\|M^{(\lambda)}(T, f)\right\|^{2}=\sum_{B^{\prime}} \lambda^{2\left|B^{\prime}\right|} \widehat{M}_{B^{\prime}}^{2}(T, f) .
$$


ЛЕмма 3.3.2. При достаточно малом $\varepsilon_{1} \lambda$ для любой функиии $f \in C_{\mathrm{b}}\left(\mathbb{R}^{\nu}\right)$ верна следующая оченка:

$$
\left\langle\mathscr{M}_{T}^{2}(f ; \eta)\right\rangle<\frac{k\|f\|_{0}}{T}
$$

где $k>0$ - константа.

ДокАзАТЕЛЬСтво. Из (3.3.1), (3.2.8a), (3.2.8b) следует представление

$$
\begin{aligned}
\widehat{M}_{B^{\prime}} & (T, f)=\prod_{b_{t} \in L^{\prime} \backslash b_{t_{f}}\left(B^{\prime}\right)} c_{1}\left(\Delta_{t}\right) \int d \Pi_{0}\left(\bar{\eta}^{(0)}\right) d \rho\left(\bar{\eta}^{(1)} \mid \bar{\eta}^{(0)}\right) \\
& \times \widehat{P}_{\bar{\eta}^{(0)}, \bar{\eta}^{(1)}}\left(s_{0}=0, x_{0}=0 ; t_{0}, y_{0}\right) \\
& \times d \rho\left(\bar{\eta}^{(2)} \mid \bar{\eta}^{(1)}\right) \widehat{P}_{\bar{\eta}^{(1)}, \bar{\eta}^{(2)}}\left(s_{1}, x_{1} ; t_{1}, y_{1}\right) \cdots d \rho\left(\eta^{k-1} \mid \eta^{k-2}\right) \\
& \times \widehat{P}_{\bar{\eta}^{(k-1)}, \bar{\eta}^{(k)}}\left(s_{k-1}, x_{k-1} ; t_{k-1}, y_{k-1}\right) G_{T, f}\left(t, \bar{y} ; \bar{\eta}^{(k)}\right) d \rho\left(\bar{\eta}^{(k)} \mid \bar{\eta}^{(k-1)}\right) .
\end{aligned}
$$

Далее заменим последний множитель в (3.3.4) на $\sup _{\bar{\eta}^{(k)}}\left|G_{T, f}\left(t, \bar{y} ; \bar{\eta}^{(k)}\right)\right|$, после чего проинтегрируем по $\bar{\eta}^{(k)}$. При этом предпоследний множитель в $(3.3 .4)$ превратится в $\widehat{P}_{\bar{\eta}^{(k-1)}}\left(s_{k-1}, x_{k-1} ; t_{k-1}, y_{k-1}\right)$. Этот множитель в свою очередь заменим на $\sup _{\bar{\eta}^{(k-1)}} \widehat{P}_{\bar{\eta}^{(k-1)}}\left(s_{k-1}, x_{k-1} ; t_{k-1}, y_{k-1}\right)$ и проинтегрируем по $\bar{\eta}^{(k-1)}$. Продолжая этот процесс дальше, мы в конце концов придем к следующему неравенству:

$$
\left|\widehat{M}_{B^{\prime}}(T, f)\right|<\prod_{b_{t} \in L^{\prime} \backslash\left\{b_{t_{f}}\left(B^{\prime}\right)\right\}}\left|c_{1}\left(\Delta_{t}\right)\right| \prod_{i=0}^{k-1} \sup _{\eta^{(i)}} \widehat{P}_{\eta^{(i)}}^{t_{i}-s_{i}}\left(y_{i} ; x_{i}\right) \sup _{\eta^{(k)}}\left|G_{T, f}\left(t, \bar{y} ; \eta^{(k)}\right)\right|
$$

где $\widehat{P}_{\bar{\eta}}^{\tau}(y ; x)=\operatorname{Pr}^{\text {уср. }}\left(X_{\tau}=y \mid X_{0}=x, \eta^{(0)}=\bar{\eta}\right)$ - вероятность для усредненной модели. Уточняя предыдущие построения (см. вывод оценки (2.3.6) и асимптотики (2.3.10)), мы докажем в приложении следующие неравенства.

ПрЕДЛОЖЕНИЕ 3.3.1. Верны следующие неравенства:

$$
\begin{gathered}
\sup _{\eta^{(k)}}\left|G_{T, f}\left(t, \bar{y} ; \eta^{(k)}\right)\right|<\frac{c_{0}}{\sqrt{T-t}}\|f\|_{0}, \\
\sum_{y}\left(\sup _{\eta} \widehat{P}_{\eta}^{t}(y ; x)\right)^{2}<\frac{c_{1}}{t^{\nu / 2}+1},
\end{gathered}
$$

где с $c_{0}$ и $c_{1}-$ константы.

Таким образом, обозначив через $\beta\left(B^{\prime}\right) \subset \mathbb{Z}^{1}$ проекцию множества $B^{\prime}$ на временную ось, получим, что

$$
\begin{aligned}
\sum_{B: \beta\left(B^{\prime}\right)=\beta}\left|\widehat{M}_{B^{\prime}}(T, f)\right|^{2} & <\left(\sum_{u} c_{1}^{2}(u)\right)^{n-1} \prod_{i=0}^{k-1} \frac{c_{0}^{2} c_{1}^{2}}{\tau_{i}^{\nu / 2}} \frac{\|f\|_{0}^{2}}{T-t} \\
& \leqslant\left(C_{2} \varepsilon_{1}^{2}\right)^{n}\|f\|_{0}^{2} c_{0}^{2 k} c_{1}^{2} \frac{1}{T-t} \prod_{i=0}^{k-1} \frac{1}{\tau_{i}^{\nu / 2}}
\end{aligned}
$$


где $n=|\beta|, \tau_{i}=t_{i}-s_{i}, i=0,1, \ldots, k-1, t=t_{f}(\beta)$ и мы воспользовались оценкой $\sum_{u} c_{1}^{2}(u)<C_{2} \varepsilon_{1}^{2}$, вытекающей из (2.1.3). Так как $\sum_{i=0}^{k-1} \tau_{i}=t-n$, мы получим, что при фиксированных $n, t$ и $k$

$$
\sum_{\substack{\tau_{0}>0, \ldots, \tau_{k-1}>0 \\ \tau_{0}+\cdots+\tau_{k-1}=t-n}} \prod_{i=0}^{k-1} \frac{1}{\tau_{i}^{\nu / 2}}<\frac{\bar{c}^{k-1}}{(t-n)^{\nu / 2}+1}
$$

где $\bar{c}>0$ - абсолютная константа. Далее, число способов представить $n$ в виде суммы из $k$ упорядоченных ненулевых слагаемых равно $C_{n-1}^{k}$. Таким образом,

$$
\sum_{\substack{B^{\prime}:\left|B^{\prime}\right|=n \\ t_{f}\left(B^{\prime}\right)=t}} \widehat{M}_{B^{\prime}}^{2}(T, f)<\left(C_{2} \varepsilon_{1}^{2}\right)^{n}\|f\|_{0}^{2} \frac{c_{1}^{2}}{(t-n)^{\nu / 2}+1} \frac{1}{T-t}\left(c_{0}^{2} \bar{c}+1\right)^{n} .
$$

Замечая, что при $C_{2} \varepsilon_{1}^{2} \lambda^{2}\left(c_{0}^{2} \bar{c}+1\right)<1$

$$
\sum_{1 \leqslant n \leqslant t} \frac{\left[C_{2} \varepsilon_{1}^{2} \lambda^{2}\left(c_{0}^{2} \bar{c}+1\right)\right]^{n}}{(t-n)^{\nu / 2}+1}<\frac{\text { const }}{t^{\nu / 2}+1}
$$

мы получим, что

$$
\begin{aligned}
\sum_{B^{\prime}} \lambda^{2\left|B^{\prime}\right|} \widehat{M}_{B^{\prime}}^{2}(T, f) & <\|f\|_{0}^{2} \text { const } \sum_{1 \leqslant t<T-1} \frac{1}{t^{\nu / 2}+1} \frac{1}{T-t} \\
& <\text { const }\|f\|_{0}^{2} \frac{1}{T} .
\end{aligned}
$$

Лемма 3.3 .2 доказана.

Рассуждая далее так же, как и в заключительной части доказательства теоремы 1.1.1, мы получим, что

$$
\left\langle\mathscr{M}_{T}^{2}\left(\mathbb{1}_{G} ; \eta\right)\right\rangle \rightarrow \infty \quad \text { при } \quad T \rightarrow \infty
$$

$\left(\mathbb{1}_{G}\right.$ - характеристическая функция области $\left.G\right)$. Соотношение (3.3.9) вместе со следствием из теоремы 2.1.1 и означает утверждение теоремы 3.1 .1 о сходимости в метрике $L_{2}$.

\section{Приложения}

А. Доказательство леммы 1.3.1. Покажем, что функции $N_{0}(\alpha)$ и $1-M_{0}(\alpha)$ (см. (1.3.10)) допускают следующие представления при $\alpha \in U \cap \Pi$, где $U$ - небольшая комплексная окрестность точки $\alpha=1$ (а $\Pi$ - напомним, комплексная плоскость с разрезом по отрезку $[1, q]$, см. с. 39$)$ :

$$
\begin{gathered}
N_{0}(\alpha)= \begin{cases}(1-\alpha)^{s-1 / 2} z^{(\nu)}(\alpha)+Z^{(\nu)}(\alpha), & \nu=2 s+1, \\
(1-\alpha)^{s} \log (1-\alpha) z^{(\nu)}(\alpha)+Z^{(\nu)}(\alpha), & \nu=2 s+2,\end{cases} \\
1-M_{0}(\alpha)= \begin{cases}(1-\alpha)^{s+1 / 2} w^{(\nu)}(\alpha)+W^{(\nu)}(\alpha), & \nu=2 s+1, \\
(1-\alpha)^{s+1} \log (1-\alpha) w^{(\nu)}(\alpha)+W^{(\nu)}(\alpha), & \nu=2 s+2 .\end{cases}
\end{gathered}
$$


Здесь $z^{(\nu)}(\alpha), Z^{(\nu)}(\alpha), w^{(\nu)}(\alpha), W^{(\nu)}(\alpha)$ - аналитические функции в окрестности $U$. При этом $z^{(\nu)}(1) \neq 0, w^{(\nu)}(1)>0,(1-\alpha)^{1 / 2}-$ ветвь, принимающая положительные значения при вещественных $\alpha<1$, a $\log (1-\alpha)$ - ветвь, принимающая вещественные значения при тех же $\alpha$. Запишем

$$
N_{0}(\alpha)=N_{0}^{(1)}(\alpha)+N_{0}^{(2)}(\alpha),
$$

где при некотором выборе $\delta>0$

$$
N_{0}^{(1)}(\alpha)=\frac{1}{\alpha} \int_{a^{2}(\lambda)<\delta} \frac{d m(\lambda)}{\beta+a^{2}(\lambda)}, \quad N_{0}^{(2)}(\alpha)=\frac{1}{\alpha} \int_{a^{2}(\lambda) \geqslant \delta} \frac{d m(\lambda)}{\beta+a^{2}(\lambda)} .
$$

Здесь обозначено: $\beta=(1-\alpha) / \alpha, a^{2}(\lambda)=1-|\hat{p}(\lambda)|^{2}$. Очевидно, что $N_{0}^{(2)}(\alpha)$ аналитична относительно $\beta$ кроме вещественной полуоси $(-\infty,-\delta)$ и, следовательно, аналитична относительно $\alpha$ при $|\alpha|<1 /(1-\delta)$. Мы выберем $\delta$ столь малым, что в окрестности

$$
G=\left\{\lambda: a^{2}(\lambda)<\delta\right\} \subset \mathbb{T}^{\nu}
$$

к функции $a^{2}(\lambda)$ применима лемма Mорса (см. [24]), т. е. в этой окрестности можно выбрать систему координат $u=\left(u_{1}, \ldots, u_{\nu}\right), u_{j}=u_{j}(\lambda), j=1, \ldots, \nu$, в которой функция $a^{2}(\lambda)$ примет вид

$$
a^{2}(\lambda)=u^{2}=u_{1}^{2}+\cdots+u_{\nu}^{2}
$$

При этом если воспользоваться разложением функции

$$
\hat{p}(\lambda)=1-\frac{1}{2} \sum_{i, j} c_{i j} \lambda_{i} \lambda_{j}+\cdots,
$$

где матрица $C=\left\{c_{i j}\right\}$ определена в (1.1.2), и четностью $|\hat{p}(\lambda)|^{2}$, то координаты $u_{1}, \ldots, u_{\nu}$ можно выбрать нечетными функциями $\lambda$ и так, что

$$
u_{i}=\sum_{k=1}^{\nu} d_{i k} \lambda_{k}+O\left(\lambda^{3}\right)
$$

где $D=\left\{d_{i k}\right\}=C^{1 / 2}$. В результате получаем, что

$$
N_{0}^{(1)}(\alpha)=\frac{1}{(2 \pi)^{\nu} \alpha} \int_{u^{2}<\delta} \frac{J(u) d u}{\beta+u^{2}},
$$

где $J(u)$ - якобиан перехода от координат $\left(\lambda_{i}, i=1, \ldots, \nu\right)$ к координатам $\left(u_{i}, i=1, \ldots, \nu\right)$ :

$$
J(u)=\operatorname{det}\left(\left(\frac{\partial u_{i}}{\partial \lambda_{j}}\right)^{-1}\right)=(\operatorname{det} C)^{-1 / 2}+O\left(u^{2}\right) .
$$

Далее, разлагая $J(u)$ в степенной ряд по переменным $\left(u_{1}, \ldots, u_{\nu}\right)$ и переходя к полярным координатам $(\rho, \theta)$, где $\theta \in \Sigma_{\nu} \subset \mathbb{R}^{\nu}$ - точка единичной сферы $\Sigma_{\nu}$ с центром в нуле, получим

$$
\int_{u^{2}<\delta} \frac{J(u)}{\beta+u^{2}} d u=\sum_{h=0}^{\infty} C_{2 h} \int_{0}^{\sqrt{\delta}} \frac{\rho^{2 h+\nu-1}}{\beta+\rho^{2}} d \rho,
$$


где при $\nu>1$

$$
\begin{gathered}
C_{0}=J(0) \omega_{\nu} \\
C_{k}=\left.\frac{1}{k} \sum_{\substack{k_{1}, \ldots, k_{\nu} \\
k_{1}+\cdots+k_{\nu}=k}} \frac{\partial^{k} J}{\partial^{k_{1}} u_{1} \cdots \partial^{k_{\nu}} u_{\nu}}\right|_{u=0} \int_{\Sigma_{\nu}} u_{1}^{k_{1}} \cdots u_{\nu}^{k_{\nu}} d \theta
\end{gathered}
$$

и $d \theta$ - элемент "площади" поверхности сферы $\Sigma_{\nu}$, а $\omega_{\nu}=\int_{\Sigma_{\nu}} d \theta$ - площадь всей сферы. При $\nu=1$ величины $C_{2 k}$ имеют вид $\frac{1}{(2 k) !} \cdot 2 \cdot J^{(2 k)}(0)$. Очевидно, что нечетные члены разложения $J(u)$ не дают вклада в сумму (А.2). Далее, для каждого целого $m \geqslant 0$ введем две функции

$$
f_{m}(\beta)=\int_{0}^{\sqrt{\delta}} \frac{\rho^{2 m}}{\beta+\rho^{2}} d \rho \quad \text { и } \quad g_{m}(\beta)=\int_{0}^{\sqrt{\delta}} \frac{\rho^{2 m+1}}{\beta+\rho^{2}} d \rho .
$$

Из легко проверяемых тождеств

$$
\begin{aligned}
\frac{\rho^{2 m}}{\rho^{2}+\beta} & =\sum_{k=0}^{m-1} \rho^{2(m-1-k)}(-\beta)^{k}+\frac{(-\beta)^{m}}{\beta+\rho^{2}}, \\
\frac{\rho^{2 m+1}}{\rho^{2}+\beta} & =\sum_{k=0}^{m-1} \rho^{2(m-1-k)+1}(-\beta)^{k}+\frac{(-\beta)^{m} \rho}{\beta+\rho^{2}}
\end{aligned}
$$

следует, что

$$
f_{m}(\beta)=P_{m-1}(\beta)+(-\beta)^{m}\left[\frac{\pi}{2 \sqrt{\beta}}-\int_{\sqrt{\delta}}^{\infty} \frac{d \rho}{\rho^{2}+\beta}\right]=F_{m}(\beta)+(-1)^{m} \frac{\pi}{2} \beta^{m-1 / 2} .
$$

Аналогично,

$$
g_{m}(\beta)=Q_{m-1}(\beta)+(-\beta)^{m} \frac{1}{2}(\log (\delta+\beta)-\log \beta)=G_{m}(\beta)+(-1)^{m+1} \frac{1}{2} \beta^{m} \log \beta,
$$

где $P_{m-1}(\beta)$ и $Q_{m-1}(\beta)$ - полиномы, а $F_{m}(\beta)$ и $G_{m}(\beta)$ - аналитические функции $\beta$ при $|\beta|<\delta$.

Таким образом, в случае нечетного $\nu=2 s+1$ получаем, что

$$
\begin{gathered}
N_{0}^{(1)}(\alpha)=\frac{1}{(2 \pi)^{\nu} \alpha} \sum_{k=0}^{\infty} C_{2 k} f_{k+s}(\beta)=\frac{1}{(2 \pi)^{\nu} \alpha} \sum_{k=0}^{\infty} C_{2 k}(-1)^{k+s} \frac{\pi}{2}\left(\frac{1-\alpha}{\alpha}\right)^{k+s-1 / 2} \\
+\sum_{k=0}^{\infty} C_{2 k} F_{k+s}\left(\frac{1-\alpha}{\alpha}\right)=(1-\alpha)^{s-1 / 2} z^{(\nu)}(\alpha)+\widetilde{Z}^{(\nu)}(\alpha),
\end{gathered}
$$

где обозначено

$$
\begin{gathered}
z^{(\nu)}(\alpha)=\frac{1}{(2 \pi)^{\nu} \alpha} \frac{\pi}{2} \sum_{k=0}^{\infty} C_{2 k}(-1)^{k+s}\left(\frac{1}{\alpha}\right)^{k+s-1 / 2}(1-\alpha)^{k} \\
\widetilde{Z}^{(\nu)}(\alpha)=\frac{1}{(2 \pi)^{\nu} \alpha} \sum_{k=0}^{\infty} C_{2 k} F_{k+s}\left(\frac{1-\alpha}{\alpha}\right)
\end{gathered}
$$


и обе эти функции аналитичны при $|\alpha-1|<\delta / 2$. Полагая

$$
Z^{(\nu)}(\alpha)=\widetilde{Z}^{(\nu)}(\alpha)+N_{0}^{(2)}(\alpha)
$$

мы и получим представление (А.1а) в случае нечетного $\nu$. При этом

$$
z^{(\nu)}(1)=\frac{\pi(-1)^{s}}{(2 \pi)^{\nu} \cdot 2} C_{0}=\frac{(-1)^{s} \pi}{2(2 \pi)^{\nu}} \omega_{\nu}(\operatorname{det} \widehat{C})^{-1 / 2} \neq 0 .
$$

Аналогично выводится представление (А.1а) в случае четного $\nu$, и при этом снова $z^{(\nu)}(1) \neq 0$.

Тем же способом выводятся представления (А.1b) для функции $1-M_{0}(\alpha)$. Из полученных представлений мы находим, что в случае нечетного $\nu=2 s+1$

$$
H_{0}(\alpha)=\frac{(1-\alpha)^{s-1 / 2} z^{(\nu)}(\alpha)+Z^{(\nu)}(\alpha)}{(1-\alpha)^{s+1 / 2} w^{(\nu)}(\alpha)+W^{(\nu)}(\alpha)}=h^{\sharp}(\alpha)(1-\alpha)^{s-1 / 2}+H^{\sharp}(\alpha),
$$

где

$$
\begin{aligned}
h^{\sharp}(\alpha) & =\frac{z^{(\nu)}(\alpha) W^{(\nu)}(\alpha)-Z^{(\nu)}(\alpha) w^{(\nu)}(\alpha)(1-\alpha)}{\left(W^{(\nu)}(\alpha)\right)^{2}-(1-\alpha)^{\nu}\left(w^{(\nu)}(\alpha)\right)^{2}}, \\
H^{\sharp}(\alpha) & =\frac{W^{(\nu)}(\alpha) Z^{(\nu)}(\alpha)-(1-\alpha)^{2 s} w^{(\nu)}(\alpha) z^{(\nu)}(\alpha)}{\left(W^{(\nu)}(\alpha)\right)^{2}-(1-\alpha)^{\nu}\left(w^{(\nu)}(\alpha)\right)^{2}}
\end{aligned}
$$

являются аналитическими функциями в малой окрестности точки $\alpha=1$, так как $W^{(\nu)}(1) \neq 0$. При этом $h^{\sharp}(1)=z^{(\nu)}(1) / W^{(\nu)}(1) \neq 0$. В случае четного $\nu=2 s+2$

$$
H_{0}(\alpha)=\frac{(1-\alpha)^{s} \log (1-\alpha) z^{(\nu)}(\alpha)+Z^{(\nu)}(\alpha)}{(1-\alpha)^{s+1} \log (1-\alpha) w^{(\nu)}(\alpha)+W^{(\nu)}(\alpha)}
$$

и мы приходим к представлению $(1.3 .14 \mathrm{~b})$, в котором

$$
h^{\sharp}(\alpha)=\frac{z^{(\nu)}(\alpha)}{W^{(\nu)}(\alpha)}, \quad H^{\sharp}(\alpha)=\frac{Z^{(\nu)}(\alpha)}{W^{(\nu)}(\alpha)}
$$

и

$$
R_{\nu}(\alpha)=-\frac{(1-\alpha)^{s+1} \log (1-\alpha) w^{(\nu)}(\alpha)\left[(1-\alpha)^{s} \log (1-\alpha) z^{(\nu)}(\alpha)+Z^{(\nu)}(\alpha)\right]}{\left[(1-\alpha)^{s+1} \log (1-\alpha) w^{(\nu)}(\alpha)+W^{(\nu)}(\alpha)\right] W^{(\nu)}(\alpha)} .
$$

Оценка (1.3.14c) для $R_{\nu}(\alpha)$ следует отсюда непосредственно. Лемма 1.3.1 доказана.

В. Доказательство леммы 2.2.1. Разложение пространства $l_{2}\left(\Gamma_{0}\right)$ в прямую сумму подпространств

$$
l_{2}\left(\Gamma_{0}\right)=\left\{c \chi_{0}\right\}+h^{0},
$$

где $\chi_{0}(\gamma)=\delta_{\gamma, \varnothing}$, а $h^{0}$ определено в $(2.2 .13 \mathrm{~b})$, порождает представление $\mathscr{T}(\lambda)$ в виде операторной матрицы

$$
\mathscr{T}(\lambda) \sim\left(\begin{array}{ll}
\mathscr{T}_{00}(\lambda) & \mathscr{T}_{01}(\lambda) \\
\mathscr{T}_{10}(\lambda) & \mathscr{T}_{11}(\lambda)
\end{array}\right),
$$


где $\mathscr{T}_{00}(\lambda):\left\{c \chi_{0}\right\} \rightarrow\left\{c \chi_{0}\right\}, \mathscr{T}_{01}(\lambda): h^{0} \rightarrow\left\{c \chi_{0}\right\}$ и т. д. При этом

$$
\begin{array}{ll}
\mathscr{T}_{00}(\lambda)\left(c \chi_{0}\right)=\left(\widehat{R}_{\varnothing, \varnothing}(\lambda) c\right) \chi_{0}, \quad\left(\mathscr{T}_{01}(\lambda) \varphi\right)_{\varnothing}=\sum_{\gamma \neq \varnothing} \widehat{R}_{\varnothing, \gamma}(\lambda) \varphi(\gamma), \quad \varphi \in h^{0}, \\
\left(\mathscr{T}_{10}(\lambda)\left(c \chi_{0}\right)\right)(\gamma)=\widehat{R}_{\gamma, \varnothing}(\lambda) c, \quad\left(\mathscr{T}_{11}(\lambda) \varphi\right)_{\gamma}=\sum_{\gamma^{\prime} \neq \varnothing} \widehat{R}_{\gamma, \gamma^{\prime}}(\lambda) \varphi\left(\gamma^{\prime}\right), \quad \varphi \in h^{0} .
\end{array}
$$

Из (2.2.7b) и (2.2.11c) следует, что

$$
\widehat{R}_{\varnothing, \varnothing}(\lambda)=\tilde{p}_{0}(\lambda)
$$

Уравнения для отыскания собственного вектора $\chi_{\lambda}$, нормированного условием $\chi_{\lambda}(\varnothing)=1$, запишутся в виде

$$
\begin{gathered}
\widehat{R}_{\varnothing, \varnothing}(\lambda)+\sum_{\gamma \neq \varnothing} \widehat{R}_{\varnothing, \gamma}(\lambda) \chi_{\lambda}(\gamma)=\tilde{p}(\lambda) \\
\widehat{R}_{\gamma, \varnothing}(\lambda)+\sum_{\gamma^{\prime} \neq \varnothing} \widehat{R}_{\gamma, \gamma^{\prime}}(\lambda) \chi_{\lambda}\left(\gamma^{\prime}\right)=\tilde{p}(\lambda) \chi_{\lambda}(\gamma) \\
=\left(\widehat{R}_{\varnothing, \varnothing}(\lambda)+\sum_{\gamma^{\prime} \neq \varnothing} \widehat{R}_{\varnothing, \gamma^{\prime}}(\lambda) \chi_{\lambda}\left(\gamma^{\prime}\right)\right) \chi_{\lambda}(\gamma)
\end{gathered}
$$

Здесь $\tilde{p}(\lambda)$ - собственное значение, соответствующее $\chi_{\lambda}$. Из (В.2) находим, что при $\gamma \neq \varnothing$

$$
\chi_{\lambda}(\gamma)=\frac{1}{\widehat{R}_{\varnothing, \varnothing}(\lambda)} \sum_{\gamma^{\prime} \neq \varnothing} \widehat{R}_{\gamma, \gamma^{\prime}}(\lambda) \chi_{\lambda}\left(\gamma^{\prime}\right)-\frac{\chi_{\lambda}(\gamma)}{\widehat{R}_{\varnothing, \varnothing}(\lambda)} \sum_{\gamma^{\prime} \neq \varnothing} \widehat{R}_{\varnothing, \gamma^{\prime}}(\lambda) \chi_{\lambda}\left(\gamma^{\prime}\right)+\frac{\widehat{R}_{\gamma, \varnothing}(\lambda)}{\widehat{R}_{\varnothing, \varnothing}(\lambda)}
$$

Обозначим $\hat{l}_{1}^{M}=l_{1}^{M} \cap h^{0}$ и рассмотрим правую часть уравнения (В.3) как отображение

$$
\mathfrak{B}_{\lambda}: \hat{l}_{1}^{M} \rightarrow \hat{l}_{1}^{M}, \quad \lambda \in O_{\delta} .
$$

ПРеДЛОЖениЕ В.1. Существует такая константа $\kappa=\kappa\left(\varepsilon_{1}, \varepsilon_{2}\right)$, что для всех $\lambda \in O_{\delta}$ сфера $S_{\kappa}=\left\{\varphi \in \hat{l}_{1}^{M}:\|\varphi\|_{M}<\kappa\right\}$ переводится отображением $\mathfrak{B}_{\lambda}$ в себя и это отображение действует в $S_{\kappa}$ сжимающим образом.

ДокАЗАТЕЛЬСтво. Вводя оператор $\widehat{R}(\lambda): \hat{l}_{1}^{M} \rightarrow \hat{l}_{1}^{M}$, действующий по формуле

$$
(\widehat{R}(\lambda) \varphi)(\gamma)=\sum_{\gamma^{\prime} \neq \varnothing} R_{\gamma, \gamma^{\prime}}(\lambda) \varphi\left(\gamma^{\prime}\right), \quad \varphi \in \hat{l}_{1}^{M}
$$

мы получим следующую оценку для $\left\|\mathfrak{B}_{\lambda} \varphi\right\|_{l_{1}^{M}}, \varphi \in \hat{l}_{1}^{M}$ :

$$
\left\|\mathfrak{B}_{\lambda} \varphi\right\|_{l_{1}^{M}} \leqslant \frac{1}{\left|\widehat{R}_{\varnothing, \varnothing}(\lambda)\right|}\left(\|\widehat{R} \cdot,(\lambda)\|_{l_{1}^{M}}+\|\widehat{R}(\lambda)\| \cdot\|\varphi\|_{l_{1}^{M}}+\max _{\gamma \neq \varnothing} \frac{\left|\widehat{R}_{\varnothing, \gamma}(\lambda)\right|}{M^{|\gamma|}}\right)
$$

при этом $\|\widehat{R}(\lambda)\|$ есть норма оператора $\widehat{R}(\lambda)$ в пространстве $l_{1}^{M}$. Из выражений (2.2.7a) и (2.2.7b) для матричных элементов $R_{\gamma, z ; \gamma^{\prime}, z^{\prime}}$ и формулы $(2.2 .11 \mathrm{c})$ 
находим, что

$$
\begin{gathered}
\|\widehat{R} \cdot, \varnothing(\lambda)\|_{l_{1}^{M}}<c_{0} \varepsilon, \\
\max _{\gamma \neq \varnothing} \frac{\left|\widehat{R}_{\varnothing, \gamma}(\lambda)\right|}{M^{|\gamma|}<c_{1} \varepsilon}, \\
\|\widehat{R}(\lambda)\|<|\mu|+\delta / 6,
\end{gathered}
$$

где $\varepsilon=\max \left(\varepsilon_{1}, \varepsilon_{2}\right)$, а $c_{0}, c_{1}$ - константы, не зависящие от $\varepsilon$. Кроме того, для $\lambda \in O_{\delta}$, как следует из (В.1b) и $(2.2 .16)$,

$$
\left|\widehat{R}_{\varnothing, \varnothing}(\lambda)\right|>1-\delta .
$$

Таким образом, из (В.4) и (В.5) получаем оценку

$$
\left\|\mathfrak{B}_{\lambda} \varphi\right\|_{l_{1}^{M}}<\frac{1}{1-\delta}\left(c_{0} \varepsilon+(\mu+\delta / 6)\|\varphi\|+c_{1} \varepsilon\|\varphi\|^{2}\right),
$$

и так как

$$
\frac{\mu+\delta / 6}{1-\delta} \equiv g<1
$$

мы можем найти такое $\kappa=\kappa(\varepsilon)$, что

$$
\frac{c_{0}}{1-\delta} \varepsilon+g \kappa+\frac{c_{1}}{1-\delta} \varepsilon \kappa^{2}<\kappa
$$

Это означает, что

$$
\mathfrak{B}_{\lambda} S_{\kappa} \subset S_{\kappa} .
$$

Аналогичным образом показывается, что $\mathfrak{B}_{\lambda}$ действует в $S_{\kappa}$ сжимающим образом. Отсюда следует, что уравнение (В.3) имеет единственное решение $\bar{\chi}_{\lambda}=$ $\left\{\bar{\chi}_{\lambda}(\gamma), \gamma \neq \varnothing\right\}$, удовлетворяющее оценке

$$
\left\|\bar{\chi}_{\lambda}\right\|_{l_{1}^{M}}=\sum_{\gamma \neq \varnothing}\left|\bar{\chi}_{\lambda}(\gamma)\right| M^{|\gamma|}<\kappa .
$$

Полагая

$$
\chi_{\lambda}(\gamma)= \begin{cases}1, & \gamma=\varnothing, \\ \bar{\chi}_{\lambda}(\gamma), & \gamma \neq \varnothing,\end{cases}
$$

мы получим собственный вектор оператора $\mathscr{T}(\lambda)$, для которого выполнено соотношение $(2.2 .19 \mathrm{a})$. Из-за четности $R_{\gamma, \gamma^{\prime}}(\lambda)$ компоненты вектора $\chi_{\lambda}(\gamma)-$ четные функции $\lambda$. Из (В.2) следует также четность $\tilde{p}(\lambda)$. Поскольку при $\gamma \neq \varnothing$ выполнено равенство $\widehat{R}_{\gamma, \varnothing}(0)=0$ то, как видно из $(2.2 .7 \mathrm{~b})$ и $(2.2 .11 \mathrm{c})$, $\chi_{\{\lambda=0\}}(\gamma)=0$ при $\gamma \neq \varnothing$, что означает (2.2.19b). Из (В.2a) и (В.5) находим, что

$$
\left|\tilde{p}(\lambda)-\tilde{p}_{0}(\lambda)\right|<c_{1} \varepsilon \kappa
$$

и, таким образом, при достаточно малом $\varepsilon$ выполнена оценка (2.2.18). Аналитичность собственного вектора $\chi_{\lambda}$, а также собственного значения $\tilde{p}(\lambda)$ относительно $\lambda \in O_{\delta}$ следует из аналитичности матричных элементов $R_{\gamma, \gamma^{\prime}}(\lambda)$. Из $(2.2 .19 \mathrm{~b}),($ В.2a) и (В.1b) следует равенство $\tilde{p}(0)=1$. Далее, покажем, что $|\tilde{p}(\lambda)| \leqslant 1$. Действительно, если в некоторой точке $\lambda_{0} \in \mathbb{T}^{\nu}$ было выполнено неравенство $\left|\tilde{p}\left(\lambda_{0}\right)\right|>1$, то это неравенство сохранялось бы и в некоторой 
окрестности $\widehat{O}$ точки $\lambda_{0}$. Пусть $\varphi(\lambda)$ - ограниченная функция с носителем в $\widehat{O}$. Очевидно, что элемент $\chi^{\varphi}$, равный $\int_{\mathbb{T}^{\nu}} \varphi(\lambda) \chi_{\lambda} d \lambda$, принадлежит пространству $\int_{\mathbb{T}^{\nu}} \mathscr{H}(\lambda) d \lambda$ и при этом выполнено соотношение

$$
\mathscr{T} \chi^{\varphi}=\int \mathscr{T}(\lambda) \varphi(\lambda) \chi_{\lambda} d \lambda=\int \tilde{p}(\lambda) \varphi(\lambda) \chi_{\lambda} d \lambda
$$

откуда видно, что

$$
\left\|\mathscr{T} \chi^{\varphi}\right\|>\left\|\chi^{\varphi}\right\|
$$

а это противоречит тому, что оператор $\mathscr{T}$ в $\mathscr{H}$ является сжимающим. Таким образом, точка $\lambda=0$ является точкой максимума функции $|\tilde{p}(\lambda)|$, которая представима в малой окрестности $\lambda=0$ в виде

$$
|\tilde{p}(\lambda)|=1-\frac{1}{2} \sum \tilde{c}_{j k} \lambda_{j} \lambda_{k}+O\left(\lambda^{3}\right),
$$

где $\left\{\tilde{c}_{j k}\right\}$ - неотрицательно-определенная матрица. Поскольку, как нетрудно вывести,

$$
\max _{j, k}\left|\tilde{c}_{j k}-c_{j k}^{0}\right|<\mathrm{const} \cdot \varepsilon,
$$

где $\left\{c_{j k}^{0}\right\}$ - положительно-определенная матрица

$$
c_{j k}^{0}=\left.\frac{\partial^{2} \tilde{p}_{0}(\lambda)}{\partial \lambda_{j} \partial \lambda_{k}}\right|_{\lambda=0},
$$

то матрица $\left\{\tilde{c}_{j k}\right\}$ при достаточно малых $\varepsilon$ также положительно определена. Далее, в силу четности $\tilde{p}(\lambda)$

$$
\arg \tilde{p}(-\lambda)=-\arg \tilde{p}(\lambda)
$$

и при малых $\lambda$

$$
\arg \tilde{p}(-\lambda)=\sum b_{k} \lambda_{k}+O\left(\lambda^{3}\right) .
$$

Представляя $\tilde{p}(\lambda)$ в виде

$$
\tilde{p}(\lambda)=|p(\lambda)| e^{i \arg \tilde{p}(\lambda)},
$$

из (В.6b) и (В.7) мы находим (2.2.20b). Представление (В.6b) означает, что неравенство $|\tilde{p}(\lambda)|<1$ выполнено в некоторой малой окрестности точки $\lambda_{0}$. Поскольку $\left|p_{0}(\lambda)\right|<1$ всюду кроме $\lambda=0$, в силу (В.6а) при малых $\varepsilon$ неравенство $|\tilde{p}(\lambda)|<1$ также выполнено всюду кроме нуля.

Итак, доказаны утверждения і) и іi) леммы 2.2.1. Далее, заметим, что оператор $\mathscr{T}^{*}$ в пространстве $\mathscr{H}$ обладает теми же свойствами, что и оператор $\mathscr{T}$, и для него также справедливо представление

$$
\mathscr{T}^{*}=\int_{\mathbb{T}^{\nu}} \mathscr{T}^{*}(\lambda) d \lambda,
$$

где $\mathscr{T}^{*}(\lambda)$ - оператор в $l_{2}\left(\Gamma_{0}\right)$, сопряженный оператору $\mathscr{T}(\lambda)$. Повторяя предыдущие рассуждения, мы получим, что у оператора $\mathscr{T}^{*}(\lambda)$ при $\lambda \in O_{\delta}$ имеется собственный вектор $\chi_{\lambda}^{*}$ с собственным значением $\overline{\tilde{p}(\lambda)}$ и при нормировке 
$\chi_{\lambda}^{*}(\varnothing)=1$ этот вектор удовлетворяет оценке (2.2.19a); кроме того, этот вектор аналитичен относительно $\lambda \in O_{\delta}$. Пространство

$$
h_{\lambda}=\left\{\varphi \in l_{1}^{M}\left(\Gamma_{0}\right):\left(\varphi, \chi_{\lambda}^{*}\right)_{l_{2}\left(\Gamma_{0}\right)}=0\right\}
$$

инвариантно относительно $\mathscr{T}(\lambda)$ и для любого вектора $\varphi \in l_{1}^{M}\left(\Gamma_{0}\right)$ справедливо разложение

$$
\varphi=c \chi_{\lambda}+\bar{\varphi}
$$

где $\bar{\varphi} \in h_{\lambda}$ и

$$
c=\frac{\left(\varphi, \chi_{\lambda}^{*}\right)_{l_{2}\left(\Gamma_{0}\right)}}{\left(\chi_{\lambda}, \chi_{\lambda}^{*}\right)_{l_{2}\left(\Gamma_{0}\right)}} .
$$

Разложение (В.8) и означает (2.2.21). Для того чтобы вывести оценку (2.2.22), заметим, что любой вектор $\Psi \in h_{\lambda}$ может быть представлен в виде

$$
\Psi=\varphi+c \chi_{\lambda},
$$

где $\varphi \in h^{0}$, а $c=c(\varphi)=-\left(\varphi, \chi_{\lambda}^{*}\right)_{l_{2}\left(\Gamma_{0}\right)} /\left(\chi_{\lambda}, \chi_{\lambda}^{*}\right)_{l_{2}\left(\Gamma_{0}\right)}$. Отсюда находим, что

$$
\|\mathscr{T}(\lambda) \Psi\|_{l_{1}^{M}\left(\Gamma_{0}\right)}<\|\mathscr{T}(\lambda) \varphi\|_{l_{1}^{M}\left(\Gamma_{0}\right)}+|c||\tilde{p}(\lambda)|\left\|\chi_{\lambda}\right\|_{l_{1}^{M}\left(\Gamma_{0}\right)} .
$$

Из оценок (В.5) получаем, что

$\|\mathscr{T}(\lambda) \varphi\|_{l_{1}^{M}\left(\Gamma_{0}\right)}<\left|\mathscr{T}_{01}(\lambda) \varphi\right|+\left\|\mathscr{T}_{11}(\lambda) \varphi\right\|_{l_{1}^{M}\left(\Gamma_{0}\right)}<c_{1} \varepsilon\|\varphi\|_{l_{1}^{M}}+\left(|\mu|+\frac{\delta}{6}\right)\|\varphi\|_{l_{1}^{M}\left(\Gamma_{0}\right)}$.

Из выражения для $c$ при $\varphi(\varnothing)=0$ следует, что

$$
|c(\varphi)|<\tilde{\delta}(\varepsilon)\|\varphi\|_{l_{1}^{M}\left(\Gamma_{0}\right)},
$$

где $\tilde{\delta}(\varepsilon)$ мало одновременно с $\varepsilon$. Наконец, из (В.9) находим, что

$$
\|\varphi\|_{l_{1}^{M}\left(\Gamma_{0}\right)}<\|\Psi\|_{l_{1}^{M}\left(\Gamma_{0}\right)}\left(1-\tilde{\delta}(\varepsilon)\left\|\chi_{\lambda}\right\|_{l_{1}^{M}\left(\Gamma_{0}\right)}\right)^{-1} .
$$

Из предыдущих оценок находим, что

$$
\|\mathscr{T}(\lambda) \Psi\|_{l_{1}^{M}\left(\Gamma_{0}\right)}<\frac{c_{1} \varepsilon+|\mu|+\delta / 6+\tilde{\delta}(\varepsilon)}{1-\tilde{\delta}(\varepsilon)\left\|\chi_{\lambda}\right\|_{l_{1}^{M}\left(\Gamma_{0}\right)}}\|\Psi\|_{l_{1}^{M}\left(\Gamma_{0}\right)} .
$$

Отсюда при достаточно малом $\varepsilon$ и следует (2.2.22). Таким образом, доказаны утверждения iii) и iv) леммы 2.2.1. Последнее утверждение леммы - оценка (2.2.23) - может быть выведена аналогичным образом с использованием неравенств (В.5). Лемма 2.2.1 доказана.

С. Доказательство леммы 3.3.1. Доказательство леммы 3.3 .1 основано на так называемой кластерной оценке семиинвариантов $\omega_{B, B^{\prime}}($ см. $(3.2 .4 \mathrm{~d}))$, а именно

$$
\left|\omega_{B, B^{\prime}}\right|<c^{\left|B \cup B^{\prime}\right|} \tau^{d_{B \cup B^{\prime}}},
$$

где $c>0$ - константа, а $\tau=\tau(\mu)$ - некоторая функция собственного значения $\mu$ матрицы $\widehat{Q}$, причем $\tau \ll 1$, если выполнено условие (3.1.1а). Число $d_{D}$, 
$D \subset \mathbb{Z}^{\nu+1}, D \neq \varnothing$, определяется как минимальная длина связного графа на решетке $\mathbb{Z}^{\nu+1}$, множество вершин которого содержит $D$ (длина графа измеряется относительно нормы $\left.|x|=\sum_{i=1}^{\nu+1}\left|x_{i}\right|, x=\left(x_{1}, \ldots, x_{\nu+1}\right)\right)$. Общее доказательство оценки (С.1) для широкого класса полей, включающего и случай нашего поля, можно найти в [23; гл. 2, п. 7]. Кроме неравенства (С.1) нам также понадобится следующая оценка.

ПреДЛОЖЕНИЕ С.1. Пусть $1>\rho>0$ - достаточно малое число. Тогда для любой фиксированной точки $x_{0} \in \mathbb{Z}^{\nu+1}$

$$
\sum_{\substack{D \subset \mathbb{Z}^{\nu+1}:|D|<\infty \\ x_{0} \in D}} \rho^{d_{D}}<1+\sum_{n=1}^{\infty}(q H(\rho))^{n}:=R(\rho)<\infty
$$

¿де

$$
q=\sup _{n \geqslant 1}\left(\frac{(n+1)^{n-1}}{n !}\right)^{1 / n}, \quad H(\rho)=\sum_{\substack{x \in \mathbb{Z}^{\nu+1} \\ x \neq 0}} \rho^{|x|} .
$$

ДоказАтельство. Очевидно, что для любого $\rho<1-\delta$, где $0<\delta<1$, справедливо неравенство

$$
H(\rho)=\sum_{\substack{x \in \mathbb{Z}^{\nu+1} \\ x \neq 0}} \rho^{|x|}<c \rho
$$

где $c=c(\nu+1, \delta)$ - некоторая константа, зависящая лишь от $\delta$ и размерности $\nu+1$. Таким образом, при условии, что $c q \rho<1$, ряд $\sum_{n=1}^{\infty}(q H(\rho))^{n}$ в $($ С.2) сходится. Далее, заметим, что

$$
\rho^{d_{D}} \leqslant \sum_{\mathscr{T}: V(\mathscr{T})=D} \prod_{(x, y) \in \mathscr{T}} \rho^{|x-y|},
$$

где сумма берется по всем деревьям $\mathscr{T}$ с множеством вершин $V(\mathscr{T})=D$, а произведение - по всем ребрам дерева $\mathscr{T}$. Таким образом,

$$
\begin{aligned}
\sum_{\substack{D \subset \mathbb{Z}^{\nu+1} \\
|D|=n+1, x_{0} \in D}} \rho^{d_{D}} & \leqslant \sum_{\substack{D \subset \mathbb{Z}^{\nu+1} \\
|D|=n+1, x_{0} \in D}} \sum_{\mathscr{T}: V(\mathscr{T})=D} \prod_{(x, y) \in \mathscr{T}} \rho^{|x-y|} \\
& =\frac{1}{n !} \sum_{\substack{\left(x_{1}, \ldots, x_{n}\right) \\
\mathscr{T}: V(\mathscr{T})=\left(x_{1}, \ldots, x_{n}\right)}} \prod_{\substack{\left(x_{i}, x_{j}\right) \in \mathscr{T} \\
i \neq j}} \rho^{\left|x_{i}-x_{j}\right|} .
\end{aligned}
$$

Здесь сумма берется по всем $n$-точечным упорядоченным наборам $\left(x_{1}, \ldots, x_{n}\right)$, $x_{i} \in \mathbb{Z}^{\nu+1}, i=1, \ldots, n$, таким, что $x_{j} \neq x_{i}, i, j=0,1, \ldots, n$, а затем по всем деревьям $\mathscr{T}$ с фиксированным множеством вершин $\left(x_{0}, x_{1}, \ldots, x_{n}\right)$, произведение берется по всем ребрам дерева $\mathscr{T}$. Переходя к абстрактным деревьям $\widehat{\mathscr{T}}$ с множеством вершин $(0,1, \ldots, n)$, мы получим, что

$$
\sum_{x_{1}, \ldots, x_{n}} \prod_{(i, j) \in \widehat{\mathscr{T}}} \rho^{\left|x_{i}-x_{j}\right|}<(H(\rho))^{n} .
$$


Действительно, пусть $0<i \leqslant n-$ концевая точка дерева $\widehat{\mathscr{T}}$. Тогда, фиксировав все точки $x_{0}, x_{1}, x_{i-1}, x_{i+1}, \ldots, x_{n}$ и суммируя по $x_{i} \in \mathbb{Z}^{\nu+1}$, мы получим, что эта сумма не превосходит $\sum_{x \in \mathbb{Z}^{\nu+1}, x \neq 0} \rho^{|x|}=H(\rho)$ и остается дерево $\widehat{\mathscr{T}}^{\prime}$ с вершинами $(0,1, \ldots, i-1, i+1, \ldots, n)$. Продолжая эту процедуру, мы и получим оценку (С.6а). Принимая теперь в расчет, что число абстрактных деревьев с $(n+1)$ вершиной $(0,1, \ldots, n)$ равно $(n+1)^{n-1}$ (см. [25]), мы из (С.5) и (С.6а) выводим неравенство (С.2). Предложение С.1 доказано.

Перейдем теперь непосредственно к доказательству леммы 3.3.1.

Имеем

$$
\begin{aligned}
\left\|\mathscr{A}^{(\lambda)}\right\|_{l_{2}\left(\Gamma_{0}\right)} & =\sup _{f, g \in l_{2}\left(\Gamma_{0}\right):\|f\|=\|g\|=1}\left|\left(\mathscr{A}^{(\lambda)} f, g\right)\right| \\
& =\sup _{f, g:\|f\|=\|g\|=1}\left|\sum_{B, B^{\prime}} A_{B, B^{\prime}} f_{B^{\prime}} \lambda^{-\left|B^{\prime}\right|} g_{B}\right| \\
& \leqslant \frac{1}{2} \sup _{f, g:\|f\|=\|g\|=1} \sum_{B, B^{\prime}} \lambda^{-\left|B^{\prime}\right|}\left|A_{B, B^{\prime}}\right|\left(\left|f_{B^{\prime}}\right|^{2}+\left|g_{B}\right|^{2}\right) \\
& \leqslant \frac{1}{2}\left(\sup _{B^{\prime}} \sum_{B} \frac{\left|A_{B, B^{\prime}}\right|}{\lambda^{\left|B^{\prime}\right|}}+\sup _{B} \sum_{B^{\prime}} \frac{\left|A_{B, B^{\prime}}\right|}{\lambda^{\left|B^{\prime}\right|}}\right) .
\end{aligned}
$$

Далее, согласно (3.2.8d) и оценке (С.1)

$$
\sum_{B}\left|A_{B, B^{\prime}}\right|<\sum_{\substack{q_{B^{\prime}} \in \varphi_{B^{\prime}} \\ q_{B^{\prime}}=\left\{B_{1}^{\prime}, \ldots, B_{s}^{\prime}\right\}}} \prod_{i=1}^{s} \sum_{B_{i}} c^{\left|B_{i}^{\prime} \cup B_{i}\right|} \tau^{d_{B_{i} \cup B_{i}^{\prime}}},
$$

где при фиксированном разбиении $q_{B^{\prime}}=\left(B_{i}^{\prime}, \ldots, B_{s}^{\prime}\right)$ множества $B^{\prime}$ сумма берется по всевозможным наборам множеств $\left\{B_{1}, \ldots, B_{s}\right\}$. Далее, поскольку $|D|<d_{D}+1$ для любого конечного множества $D \subset Z^{\nu+1}$,

$$
c^{|D|} \tau^{d_{D}}<c \tau_{*}^{d_{D}}
$$

где $\tau_{*}=c \tau$.

Фиксировав число $s=\left|q_{B^{\prime}}\right|$, мы просуммируем в (С.7а) по всем разбиениям $q_{B^{\prime}}=\left(B_{1}^{\prime}, \ldots, B_{s}^{\prime}\right)$ таким, что каждое множество $B_{i}^{\prime}$ содержит фиксированную точку $x_{i}, i=1, \ldots, s$. Получим

$$
\sum_{\substack{q_{B^{\prime}}=\left\{B_{1}^{\prime}, \ldots, B_{s}^{\prime}\right\} \\ x_{i} \in B_{i}^{\prime}, i=1, \ldots, s}} \prod_{j=1}^{s} \sum_{B_{j}} c^{\left|B_{j} \cup B_{j}^{\prime}\right|} \tau^{d_{B_{j}^{\prime} \cup B_{j}}<c^{s}} \prod_{i=1}^{s} \sum_{D_{i}: x \in D_{i}} \tau_{*}^{d_{D}}<\left(c R\left(\tau_{*}\right)\right)^{s} .
$$

Учитывая всевозможные способы выбрать $s$ точек из множества $B^{\prime}$, найдем, что правая часть (С.7а) оценивается величиной

$$
\sum_{s=1}^{\left|B^{\prime}\right|} C_{\left|B^{\prime}\right|}^{s}\left(c R\left(\tau^{*}\right)\right)^{s} \leqslant\left(1+c R\left(\tau^{*}\right)\right)^{\left|B^{\prime}\right|} .
$$

Таким образом, для достаточно больших $\lambda$

$$
\sup _{B^{\prime}} \sum_{B} \frac{\left|A_{B, B^{\prime}}\right|}{\lambda^{\left|B^{\prime}\right|}}<\sup _{B^{\prime}}\left(\frac{1+c R\left(\tau^{*}\right)}{\lambda}\right)^{\left|B^{\prime}\right|}<1 .
$$


Перейдем ко второму слагаемому в правой части (C.6b):

$$
\sup _{B} \sum_{B^{\prime}} \frac{\left|A_{B, B^{\prime}}\right|}{\lambda^{\left|B^{\prime}\right|}}
$$

Предварительно заметим, что

$$
\tau_{*}^{d_{D}} \leqslant \tau_{*}^{\frac{1}{2} d_{D}} \tau_{*}^{\frac{1}{2}(|D|-1)} \leqslant \begin{cases}\tau_{*}^{\frac{1}{4}|D|+\frac{1}{2} d_{D}}, & |D|>1 \\ 1, & |D|=1\end{cases}
$$

Рассуждая как и выше, получим оценку

$$
\begin{gathered}
\sum_{\substack{q_{B}=\left(B_{1}, \ldots, B_{s}\right) \\
x_{i} \in B_{i}, i=1, \ldots, s}} \prod_{j=1}^{s} \sum_{B_{j}^{\prime}} c^{\left|B_{j}^{\prime} \cup B_{j}\right|} \tau^{d_{B_{j}^{\prime} \cup B_{j}}} \lambda^{-\left|B_{j}^{\prime}\right|} \\
<c^{s} \sum_{\substack{q_{B}=\left(B_{1}, \ldots, B_{s}\right) \\
x_{i} \in B_{i}, i=1, \ldots, s}} \prod_{j=1}^{s} \sum_{B_{j}^{\prime}} \tau_{*}^{d_{B_{j} \cup B_{j}^{\prime}}} \lambda^{-\left|B_{j}^{\prime}\right|} .
\end{gathered}
$$

Воспользовавшись (С.8b), мы найдем, что правая часть последнего неравенства не превосходит величину

$$
\tau_{*}^{|B|} c^{s} \sum_{\substack{q_{B}=\left(B_{1}, \ldots, B_{s}\right) \\ x_{i} \in B_{i}, i=1, \ldots, s}} \prod_{j=1}^{s} \sum_{B_{j}^{\prime}} \tau_{*}^{\frac{1}{2} d_{B_{j} \cup B_{j}^{\prime}}}
$$

Снова рассуждая как и выше, получим, что эта величина не больше, чем

$$
\tau_{*}^{\frac{1}{4}|B|}\left(c R\left(\sqrt{\tau_{*}}\right)\right)^{s} .
$$

Окончательно получаем, что

$$
\sup _{B} \sum_{B^{\prime}} \frac{\left|A_{B, B^{\prime}}\right|}{\lambda^{\left|B^{\prime}\right|}} \leqslant \sup _{B} \tau_{*}^{\frac{1}{4}|B|}\left(1+c R\left(\sqrt{\tau_{*}}\right)\right)^{|B|}<1 .
$$

Отсюда, из (С.8a) и (C.6b) вытекает утверждение леммы. Лемма 3.3.1 доказана.

D. Доказательство предложения 3.3.1. Рассуждая так же, как и при доказательстве теоремы 2.1.1, и учитывая, что в силу условия (3.1.1b) окрестность $O_{\delta}$ при $\delta=1-2 \mu$ совпадает со всем тором $\mathbb{T}^{\nu}$, мы придем к следующему представлению для вероятности $\widehat{P}_{\eta}^{t}(y ; x)$ :

$$
\widehat{P}_{\eta}^{t}(y ; x)=\int_{\mathbb{T}^{\nu}} e^{-i(\lambda, y-x)}\left[\tilde{p}^{t}(\lambda)\left(1+g_{\eta-x}(\lambda)\right)+\bar{g}_{\eta-x}(\lambda, t)\right] d \lambda,
$$

где $\tilde{p}(\lambda)$ - старшее собственное значение оператора $\mathscr{T}(\lambda)$ (см. лемму 2.2.1), которое вместе с функциями $g_{\eta}(\lambda)$ и $\bar{g}_{\eta}(\lambda ; t)$ является аналитическим в некоторой комплексной $d$-окрестности тора

$$
W_{d}^{\nu}=\left\{\lambda \in \mathbb{C}^{\nu} / \mathbb{Z}^{\nu}: \operatorname{Re} \lambda \in \mathbb{T}^{\nu},|\operatorname{Im} \lambda|<d\right\} .
$$


При этом

i) $g_{\eta}(0)=\bar{g}_{\eta}(0, t)=0$,

ii) функции $g_{\eta}(\lambda)$ и $e^{\alpha t} g_{\eta}(\lambda, t)$ ограничены в $W_{d}^{\nu}$ равномерно по $\eta$ и $t$ для некоторого $\alpha>0$.

Из представления (D.1) стандартным способом с помощью метода перевала (см. [6] и [14]) можно вывести следующие асимптотические разложения: при любом целом $r=1,2, \ldots$

$$
\begin{aligned}
\widehat{P}_{\eta}^{t}(y ; 0)= & \frac{c^{1 / 2}}{(2 \pi t)^{\nu / 2}} \exp \left\{-\frac{1}{2} \mathscr{A}\left(\frac{y-b t}{\sqrt{t}}\right)\right\} \\
& \times\left(1+\sum_{j=1}^{r} \frac{1}{t^{j / 2}} Q_{\eta}^{j}\left(\frac{y-b t}{\sqrt{t}}\right)\right)+\frac{\delta_{r}(t)}{t^{\nu / 2}} \Delta_{\eta, t}^{(r)}\left(\frac{y-b t}{\sqrt{t}}\right)
\end{aligned}
$$

и аналогично этому

$$
\begin{aligned}
h_{\eta}^{t}(y ; 0)= & \frac{c^{1 / 2}}{(2 \pi t)^{\nu / 2}} \exp \left\{-\frac{1}{2} \mathscr{A}\left(\frac{y-b t}{\sqrt{t}}\right)\right\} \\
& \times\left(\frac{1}{\sqrt{t}} \bar{Q}_{\eta}^{1}\left(\frac{y-b t}{\sqrt{t}}\right)+\sum_{j=2}^{r} \frac{1}{t^{j / 2}} \bar{Q}_{\eta}^{j}\left(\frac{y-b t}{\sqrt{t}}\right)\right)+\frac{\bar{\delta}_{r}(t)}{t} \bar{\Delta}_{\eta, t}^{(r)}\left(\frac{y-b t}{\sqrt{t}}\right) .
\end{aligned}
$$

Здесь $\mathscr{A}(u)$ - квадратичная форма с матрицей $C\left(\right.$ см. (1.1.2)), $c=(\operatorname{det} C)^{-1}$. Функции $Q_{\eta}^{j}(u), j \geqslant 1$, и $\bar{Q}_{\eta}^{j}(u), j \geqslant 2$, являются полиномами от $u \in \mathbb{R}^{\nu}$ степени $3 j$ с коэффициентами, зависящими от $\eta$, причем эти коэффициенты ограниченные функции $\eta, \bar{Q}_{\eta}^{1}(u)$ - линейная функция $u \in \mathbb{R}^{\nu}$. Далее,

$$
\begin{array}{cl}
\delta_{r}(t)=o\left(t^{-r / 2}\right), & \bar{\delta}_{r}(t)=o\left(t^{-r / 2}\right), \\
\left|\Delta_{\eta, t}^{(r)}(u)\right|<\frac{\mathrm{const}}{1+|u|^{2+r}}, & \left|\bar{\Delta}_{\eta, t}^{(r)}(u)\right|<\frac{\mathrm{const}}{1+|u|^{2+r}},
\end{array}
$$

где константы не зависят от $\eta$ и $t$. Из приведенного разложения для $\widehat{P}_{\eta}^{t}(y ; 0)$ следует, что при $r=\nu$ величина $\sup _{\eta} \widehat{P}_{\eta}^{t}(y ; 0)$ оценивается выражением, аналогичным правой части (D.2), в котором полиномы $Q_{\eta}^{j}(u)$ заменены функциями $\sup _{\eta}\left|Q_{\eta}^{j}(u)\right|$, являющимися полиномами степени $3 j$ от переменных $\left\{\left|u_{i}\right|, i=\right.$ $1, \ldots, \nu\}$, а $\Delta_{\eta, t}^{(\nu)}(u)$ заменяется на $\frac{\text { const }}{1+|u|^{r+\nu}}$. Из этой оценки уже непосредственно следует неравенство (3.3.6b). Неравенство (3.3.6а) для $\sup _{\eta}\left|G_{T, f}(t, \bar{y} ; \eta)\right|$ подобным же образом выводится из представления (D.3) для $h_{\eta}^{t}(y ; 0)$. Предложение 3.3.1 доказано.

\section{Список литературы}

[1] O. Zeitouni, "Random walks in random environment", Lectures on probability theory and statistics (Saint-Flour, 2001), Lecture Notes in Math., 1837, ed. J. Picard, Springer, Berlin, 2004, 189-312.

[2] F. Rassoul-Agha, T. Seppalainen, "An almost sure invariance principle for random walks in a space-time random environment", Probab. Theory Related Fields, 133:3 (2005), 299-314. 
[3] J. Bérard, "The almost sure central limit theorem for one-dimensional nearest-neighbour random walks in a space-time random environment", J. Appl. Probab., 41:1 (2004), 83-92.

[4] A. Bandyopadhyay, O. Zeitouni, "Random walk in dynamic Markovian random environment", ALEA Lat. Amer. J. Probab. Math. Stat., 1 (2006), 205-224.

[5] C. Boldrighini, R. A. Minlos, A. Pellegrinotti, "Almost-sure central limit theorem for directed polymers and random corrections", Comm. Math. Phys., 189:2 (1997), 533-557.

[6] И. И. Гихман, А. В. Скороход, Теория случайных процессов, т. 1, Наука, М., 1971; англ. пер.: I. I. Gihman, A. V. Skorohod, The theory of stochastic processes, Vol. 1, Grundlehren Math. Wiss., 210, Springer, Berlin, 1974.

[7] C. Boldrighini, R. A. Minlos, A. Pellegrinotti, "Random walks in quenched i.i.d. space-time random environment are always a.s. diffusive", Probab. Theory Related Fields, 129:1 (2004), 133-156.

[8] C. Boldrighini, R. A. Minlos, A. Pellegrinotti, "Almost-sure central limit theorem for a Markov model of random walk in dynamical environment", Probab. Theory Related Fields, 109:2 (1997), 245-273.

[9] M. S. Barnabei, C. Boldrighini, R. A. Minlos, A. Pellegrinotti, "Almost-sure central limit theorem for a model of random walk in fluctuating random environment", Markov Process. Related Fields, 4:3 (1998), 381-394.

[10] C. Boldrighini, A. Pellegrinotti, " $T^{-1 / 4}$-noise for random walks in dynamic environment on $\mathbb{Z} "$, Mosc. Math. J., 1:3 (2001), 365-380.

[11] M.S. Bernabei, "Anomalous behaviour for random corrections to the cumulants of random walks in fluctuating random media", Probab. Theory Related Fields, 119:3 (2001), 410-432.

[12] C. Boldrighini, R. A. Minlos, A. Pellegrinotti, "Central limit theorem for a random walk in dynamical environment: integral and local", Theory Stoch. Process., 5(21):3-4 (1999), 16-28.

[13] М.Ш. Бирман, М.З. Соломяк, Спектралъная теория самосопряженных операторов в гилъбертовом пространстве, Изд-во ЛГУ, Л., 1980; англ. пер.: M.S. Birman, M.Z. Solomyak, Spectral theory of self-adjoint operators in Hilbert space, Math. Appl. (Soviet Ser.), 5, Kluwer, Dordrecht, 1987.

[14] М. В. Федорюк, Асимптотика: интеграль и рядъ, Справочная матем. библиотека, Наука, М., 1987.

[15] C. Boldrighini, I. A. Ignatyuk, V. A. Malyshev, A. P. Pellegrinotti, "Random walk in dynamic environment with mutual influence", Stochastic Process. Appl., 41:1 (1992), 157-177.

[16] C. Boldrighini, R. A. Minlos, A. Pellegrinotti, "Central limit theorem for the random walk of one and two particles in a random environment, with mutual interaction", Probability contributions to statistical mechanics, Adv. Soviet Math., 20, Amer. Math. Soc., Providence, 1994, 26-75.

[17] C. Boldrighini, R. A. Minlos, A. Pellegrinotti, "Interacting random walk in a dynamical random environment. I: Decay of correlations", Ann. Inst. H. Poincaré Probab. Statist., 30:4 (1994), 519-558; "II: Environment from the point of view of the particle", 559-605.

[18] C. Boldrighini, R. A. Minlos, A. Pellegrinotti, "Asymptotic decay of correlations for fluctuating environment in interacting with two-particle random walk", Boll. Un. Mat. Ital. B (7), 10:2 (1996), 277-302.

[19] C. Boldrighini, R. A. Minlos, F. R. Nardi, A. Pellegrinotti, "Asymptotic decay of correlations for a random walk in interaction with a Markov field", Mosc. Math. J., 5:3 (2005), 507-522. 
[20] I. Ignatiouk-Robert, "Large deviations for a random walk in dynamical random environment", Ann. Inst. H. Poincaré Probab. Statist., 34:5 (1998), 601-636.

[21] C. Boldrighini, R. A. Minlos, A. Pellegrinotti, "Random walk in fluctuating random environment with Markov evolution", On Dobrushin's way. From probability theory to statistical physics, Amer. Math. Soc. Transl. Ser. 2, 198, Amer. Math. Soc., Providence, 2000, 13-35.

[22] В.А. Малышев, Р.А. Минлос, Линейнъе операторы в бесконечночастичных системах, Наука, М., 1994; англ. пер.: V.A. Malyshev, R. A. Minlos, Linear infinite-particle operators, Transl. Math. Monogr., 143, Amer. Math. Soc., Providence, 1995.

[23] В.А. Малышев, Р.А. Минлос, Гиббсовские случайнъе поля. Метод кластерных разложений, Наука, M., 1985; англ. пер.: V.A. Malyshev, R A. Minlos, Gibbs random fields. Cluster expansions, Math. Appl. (Soviet Ser.), 44, Dordrecht, Kluwer, 1991.

[24] Дж. Милнор, Теория Морса, Мир, М., 1965; пер. с англ.: J. Milnor, Morse theory, Ann. Math. Stud., 51, Princeton Univ. Press, Princeton, 1963.

[25] О. Оре, Теория графов, Наука, М., 1980; пер. с англ.: O. Ore, Theory of graphs, Amer. Math. Soc. Colloq. Publ., 38, Amer. Math. Soc., Providence, 1962.

К. Болдригини (K. Boldrigini)

Università di Roma "La Sapienza", Roma, Italia

E-mail: boldrigh@mat.uniroma1.it

P. А. Минлос (R. А. Minlos)

Институт проблем передачи информации

им. А. А. Харкевича РАН

E-mail: minl@iitp.ru

\section{А. Пеллегринотти (А. Pellegrinotti)}

Università degli Studi Roma Tre, Roma, Italia

E-mail: pellegri@mat.uniroma3.it
Поступила в редакцию

27.03.2007 